Florida International University FIU Digital Commons

\title{
The Longitudinal Influence of Physical Activity on Adolescent Alcohol Use
}

Beverly A. Ruffin

Florida International University, bruff001@fiu.edu

DOI: $10.25148 /$ etd.FI12050213

Follow this and additional works at: https://digitalcommons.fiu.edu/etd

\section{Recommended Citation}

Ruffin, Beverly A., "The Longitudinal Influence of Physical Activity on Adolescent Alcohol Use" (2012). FIU Electronic Theses and Dissertations. 604.

https://digitalcommons.fiu.edu/etd/604 


\section{FLORIDA INTERNATIONAL UNIVERSITY}

Miami, Florida

THE LONGITUDINAL INFLUENCE OF PHYSICAL ACTIVITY ON ADOLESCENT

ALCOHOL USE

A dissertation submitted in partial fulfillment of

the requirements for the degree of

DOCTOR OF PHILOSOPHY

in

SOCIAL WELFARE

by

Beverly A. Ruffin

2012 
To: Interim Dean Michele Ciccazzo

R.Stempel College of Public Health and Social Work

This dissertation, written by Beverly A. Ruffin, and entitled The Longitudinal Influence of Physical Activity on Adolescent Alcohol Use, having been approved in respect to style and intellectual content, is referred to you for judgment.

We have read this dissertation and recommend that it be approved.

Mario De La Rosa

Mark Macgowan

James Jaccard

Eric Wagner, Major Professor

Date of Defense: March 21, 2012

The dissertation of Beverly A. Ruffin is approved.

Interim Dean Michele Ciccazzo

R.Stempel College of Public Health and Social Work

Dean Lakshmi N. Reddi

University Graduate School

Florida International University, 2012 
(C) Copyright 2012 by Beverly A. Ruffin

All rights reserved. 


\section{DEDICATION}

I have to take a moment to dedicate this piece of work to those who have been most supportive over the last four years. I have dedicated this dissertation first and foremost to my parents. Without their steadfast and continued love, encouragement, understanding, and support, the completion of this work would not have been possible. The both of you have instilled such a drive within me that I truly know now that anything is within my reach with hard work and dedication. I will forever be grateful for all you have done for me and what exemplary role models you both have been and continue to be throughout my life. I have also dedicated this dissertation to three little and powerful people in my life; John Carter, Angela Marie, and Justin Charles, words can't express how much your Nanna loves you and how being away from you during this process was a challenge for me. However, your simple conversations and laughter kept me extremely focused on my goal. This is dedicated to you all; to your hopes and your dreams. I can only hope that I have inspired in you what my parents have in me and that is to never give up on your dreams and to work hard at everything that you do. To my sister and brother-n-law, you paved the way for me and set a wonderful road map to follow to reach success in life. You were always there for support when things seemed un-surmountable. Each and every time you saw me through. To the rest of my family this work is a testament to you all and dedicated to each and every one of you. 


\section{ACKNOWLEDGMENTS}

There are so many people to whom I owe a great deal of thanks for their wisdom, support, and guidance during my Ph.D. study. First and foremost, I would like to thank my chair, Dr. Eric Wagner. You have been such a source of inspiration, knowledge, motivation, and support for me through this process. You offered me valuable advice, ideas, and suggestions. Most importantly, you offered me gentle yet firm criticisms just when needed. I have an undeniable appreciation for your profound knowledge and rich research experiences that I have been so blessed to be a witness to. I also offer a sincere and special thanks to Dr. James Jaccard for his tremendous statistical expertise, help and support in the accomplishment of this work. The accomplishments of my dissertation are also due, in a large part, to the support from other members of my great committee: Dr. Mario De La Rosa and Dr. Mark Macgowan. I truly appreciate them for their knowledge, experiences, and unselfish support. I could never be more proud of my committee. Without their knowledge, perceptiveness and guidance, I would never have finished this research. Furthermore, I would like to thank Yasmin Rey for her invaluable assistance support and encouragement given during the analysis phase of my dissertation.

Words, again, can't express my appreciation to my colleagues and research support staff from the Center of Research on Latino AIDS/HIV and Drug Abuse (CRUSADA) who have helped me through the last four years. I was extremely lucky to be a doctoral trainee at CRUSADA. The resources have been invaluable and many of my skills and knowledge have been shaped as a result of being a part of such a diverse group of researchers, students, and administrative staff. Particularly, I acknowledge the assistance and support from Rosa Babino and Samuel Martinez. 
Again to my family and my invaluable network of loving, supportive, and patient friends I could not have survived this process without you. Special thanks to Mariana Sanchez, Adelaida Rosario, and Elena Cyrus. Also, I can't forget the immense support from my friends outside of school. I could not have done this without you either.

I would like to also acknowledge the financial support I received from the National Institute on Alcohol Abuse and Alcoholism in the form of a Ruth L. Kirschstein National Research Service Awards for Individual Pre-doctoral Fellows.

In addition, this research uses data from The National Longitudinal Study of Adolescent Health (Add Health), a program project designed by J. Richard Udry, Peter S. Bearman, and Kathleen Mullan Harris. The project was funded by a grant P01-HD31921 from the Eunice Kennedy Shriver National Institute of Child Health and Human Development (NICHD), along with cooperative funding from 17 other agencies. I acknowledge the Inter-University Consortium for Political and Social Research in Ann Arbor, Michigan for preparing and providing the data for public use.

And to FIU and the Robert Stempel College of Public Health and Social Work, this has been the most stimulating, challenging, and rewarding environment I have ever been a part of. I am more than proud to have studied at this institution and received my degree from FIU. I have been surrounded by the most incredible researchers and the most diverse of backgrounds and every aspect of this experience and this institution have shaped the researcher I strive to be. My life has truly been enriched as result of my move to Miami and my attendance and completion of my degree at Florida International University. 


\section{ABSTRACT OF THE DISSERTATION \\ THE LONGITUDINAL INFLUENCE OF PHYSICAL ACTIVITY ON ADOLESCENT \\ ALCOHOL USE}

by

Beverly A. Ruffin

Florida International University, 2012

Miami, Florida

\section{Professor Eric Wagner, Major Professor}

The purpose of this research was to explore the influence of physical activity on depressive symptomatology and adolescent alcohol use during an underexplored transition from middle school to high school. The study initiative is supported by the fact that research has shown a unique and simultaneous decrease in physical activity (CDC, 2010), increase in depressive symptomatology (SAMHSA, 2010) and increase in alcohol use (USDHHS, 2011) during middle adolescence. A risk and resilience framework was used in efforts to conceptualize how these variables may be inter-related.

Data from waves I and II of the National Longitudinal Study of Adolescent Health (Add Health, Bearman et al., 1997; Udry, 1997) was used ( $=2,054$; aged 13-15 years). The sample was ethnically and racially diverse (58.2\% White, $24 \%$ African American, $11.7 \%$ Hispanic, and $6.1 \%$ other). Structural equation models were developed to test the potential influence physical activity has on adolescent alcohol use (e.g., frequency of alcohol use and binge alcohol use) and whether any of the relationship was mediated by depressive symptomatology or varied as a function of gender. 
Results demonstrated that there was a significant influence of structured physical activity (e.g., sports) on adolescent alcohol use. However, contrary to the proposed hypothesis, engaging in structured physical activity appeared to contribute to greater binge drinking among adolescents. Instead of demonstrating a protective feature, the findings suggest that engaging in structured physical activity places adolescents at risk for binge drinking. Furthermore, no significant relationships, positive or negative, were found for the influence of physical activity (structured and unstructured) on frequency of alcohol use. The findings regarding mediation revealed binge drinking as a mediator between physical activity (structured) and depressive symptomatology. These findings provide support for research, practice, and policy initiatives focused on developing a more comprehensive understanding of alcohol use drinking behaviors, physical activity involvement, and depressive symptomatology among adolescents, which this study demonstrates are all associated with one another. Results represent an initial step toward evaluating these relationships at a much younger age. 


\section{TABLE OF CONTENTS}

CHAPTER

PAGE

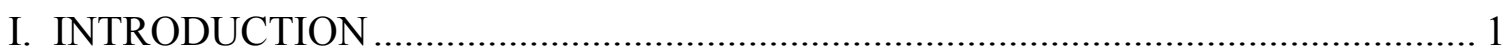

Adolescent Alcohol Use During Middle Adolescence .................................................... 2

Physical Activity: Operationalization, Inactivity Patterns, and Research Links to

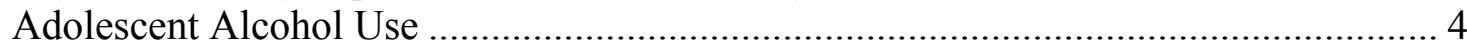

Depressive Symptomatology: A Mediation Approach ............................................... 7

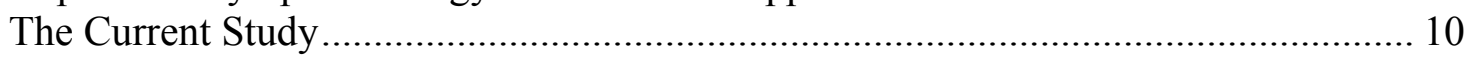

II. REVIEW OF THE LITERATURE................................................................. 12

Population of Focus: Adolescents .......................................................................... 12

Transition from Middle School to High School: Middle Adolescence ........................ 15

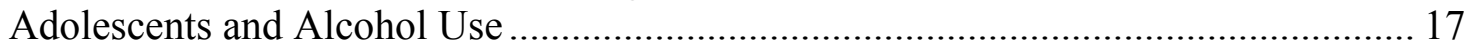

Various Indicators in Adolescent Alcohol Use and Alcohol Use Vulnerability .......... 26

Depression during Adolescence............................................................................. 28

Depression and Alcohol Use during Adolescence....................................................... 34

Linking Patterns of Physical Activity Participation to Alcohol Use ............................ 36

Extracurricular Activities: Advantageous to Youth Development ................................ 37

Adolescents and Physical Activity...................................................................... 38

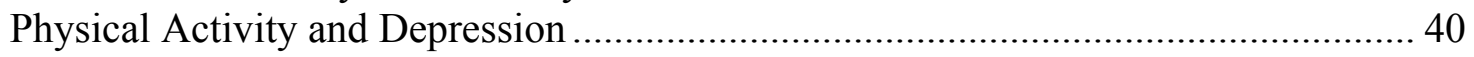

Physical Activity Participation and Alcohol Use........................................................ 41

Review of Studies on Physical Activity Participation and Alcohol Use: Variability in

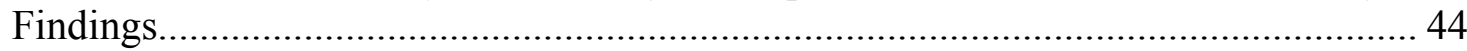

Theoretical Framework for Current Study ................................................................ 47



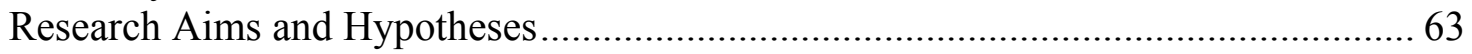



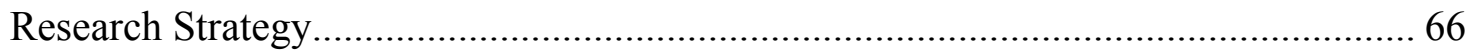

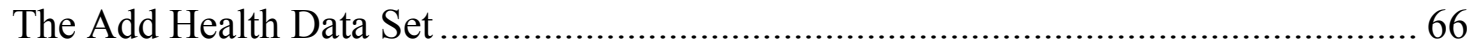

Add Health Data Participants and Collection Procedures ............................................ 68

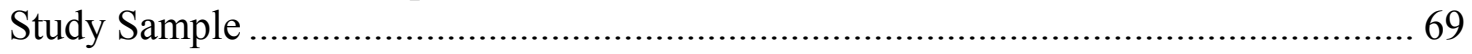

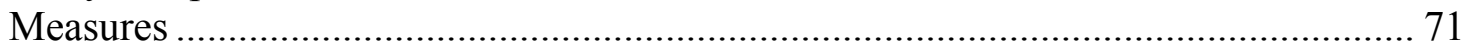

Summary of Analysis Plan................................................................................ 74



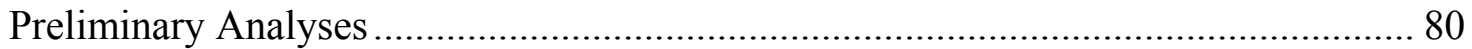

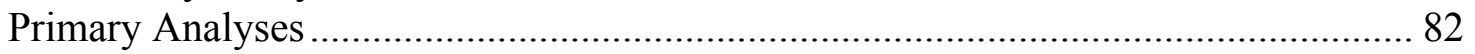

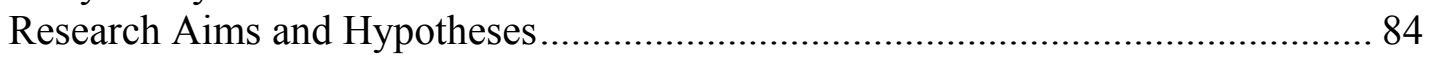

Results for Frequency of Alcohol Use Models ..................................................... 84

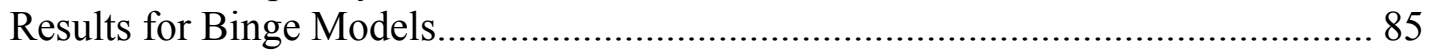

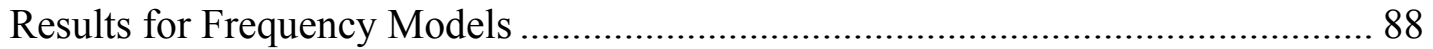

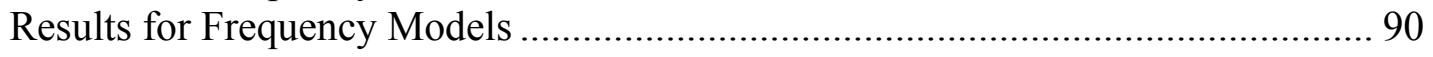

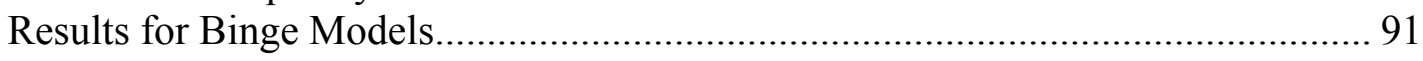




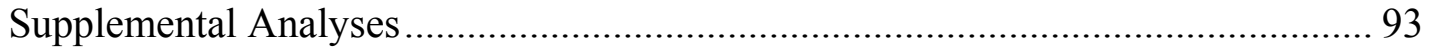

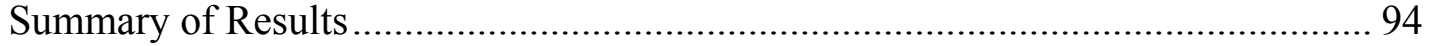







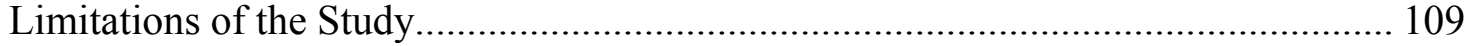



Implications for Social Work Research, Practice and Policy .................................. 113

Conclusions and Future Directions.................................................................... 121



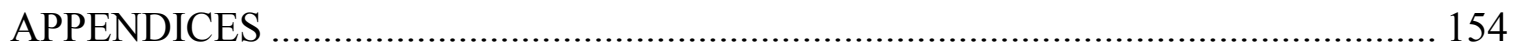

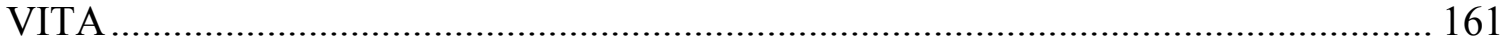


1. Demographic Information Reported in Frequencies, Standard Deviations and Errors for Add Health Study Participants.....................................

2. Means, Standard Deviations and Errors of Study Variables both Unweighted and Weighted for Total Sample and by Gender....

3. Skewness and Kurtosis for Study Variables

4. Path Coefficients, $p$ values and 95\% Confidence Intervals for SEM Model with Frequency as Outcome for Males.................................

5. Path Coefficients, $p$ values and 95\% Confidence Intervals for SEM Model with Frequency as Outcome for Females...............................

6. Path Coefficients, $p$ values and 95\% Confidence Intervals for SEM Model with Binge Drinking as Outcome for Males..............................

7. Path Coefficients, $p$ values and 95\% Confidence Intervals for SEM Model with Binge Drinking as Outcome for Females. 


\section{LIST OF FIGURES}

FIGURE

PAGE

1. Conceptual Model with Frequency of Alcohol Use as Outcome

2. Conceptual Model with Binge Drinking as Outcome .......................................... 132

3. Model with Frequency of Alcohol Use as Outcome for Males .............................. 133

4. Model with Frequency of Alcohol Use as Outcome for Females............................ 134

5. Model with Frequency of Binge Drinking as Outcome for Males 135

6. Model with Frequency of Binge Drinking as Outcome for Females 136 


\section{CHAPTER I. INTRODUCTION}

Adolescence, characterized as the period between childhood and adulthood, is a unique period in the life span that presents opportunities and challenges in improving and maintaining health (Mulye et al., 2009). One developmental milestone and purpose of adolescence is preparation for adulthood. During adolescence, young people are becoming increasingly more independent and taking greater initiative and responsibility for habits such as exercise, diet, substance use and sexual risk behaviors. As a result, the period is marked with the development of a variety of health promoting and healthcompromising behaviors.

The health status of youth in the United States have been discussed in numerous reports, concluding that the predominate threat to adolescents' health are the health-risk behaviors and decisions adolescents make in regards to health-related behaviors (Resnick et al., 1997; CDC, 2004; Mulye et al., 2009; USDHHS, 2010). Learned early, bad habits and the consequences of unhealthy choices can have profound and lasting effect on the future of Americans (USDHHS, 2007). Knowledge of how adolescents become involved in negative health behaviors, and ways to protect adolescents from these harmful influences is essential to the field of adolescent research.

This particular study will focus on two behaviors, one health- compromising and one health-promoting, alcohol use and physical activity, respectively. In respect to these two variables, the role of depressive symptomatology as a plausible mediator will be closely examined. All of these variables play a role during adolescence. However, the ability to examine the interplay among these variables during middle adolescence where there is a simultaneous increase in the onset and frequency of drinking (Chassin, Flora, \& 
King, 2004; Faden, 2006; Johnston, O’Malley, Bachman, \& Schulenberg, 2009), declines in physical activity (National Center for Health Statistics, 2005; Singh, Mahashwari, Sharma, \& Anand, 2006; Center for Disease Control \& Prevention (CDC), 2008), and increases in depressive symptomatology (SAMSHA, 2008) is particularly compelling.

\section{Adolescent Alcohol Use During Middle Adolescence}

The use of alcohol is very common in our society. Despite declines in overall alcohol consumption over the past three decades, alcohol has been and continues to be an integral part of American life (U.S. Department of Health and Human Services (USDHHS), 2007). According to a recent national survey (SAMHSA, 2008), slightly more than half of young persons aged 12 or older report being current drinkers of alcohol. More specifically, adolescent alcohol use is a pressing social and public health concern. In a recent SAMHSA report (2010), it was reported that almost 8,000 adolescents, ages 12 to 17 , reported drinking alcohol for the first time. Furthermore, about 18 percent of $8^{\text {th }}$ graders and 41.2 percent of $10^{\text {th }}$ graders have been drunk at least once (Johnston, O'Malley, Bachman, \& Schulenberg, 2007). By the time U.S. children are seniors in high school, about 30 percent are drinking at least once a month. In addition to the early initiation of alcohol consumption, when young people drink, they tend to drink in large quantities, often consuming four to five drinks at one time (Johnston, O'Malley, Bachman, \& Schulenberg, 2008).

The age range for adolescence as defined by the Centers for Disease Control includes 10-19 years of age (CDC, 2008). However, in particular, this study closely examined middle-aged adolescence and an under explored transitional period from middle school to high school (e.g., 13-15 years of age) because of the contemporaneous 
declines in health promoting behaviors and increases in health compromising behaviors (Wagner, 2008; USDHHS, 2009) during this specific developmental stage. Due to this compelling overlap there exists a real necessity to conduct research and discover methods that will prevent and promote the initiation of negative and positive health behaviors respectively.

It is well known that initiation and use of alcohol becomes more salient and common during adolescence (Johnston et al., 2009). However, what is unique and lends support to focusing on middle adolescence is that studies show increases in initiation and use of alcohol during this transitional period (Faden, 2006; SAMSHA, 2008; Johnston et al., 2008). At the same time, particularly specific to overall substance use research, most has focused on late adolescence and the transition from high school to college. However, the transition out of middle school into high school is a critical passage in life. For example, there exists a paucity of children between the ages of 10 and 15 years of age in treatment studies. This is troublesome due to the fact that distinct behavioral differences in the patterns and use of alcohol begin to develop in middle school (Wagner, 2008). According to Guo, Collins, Hill, \& Hawkins (2000) the development of these distinct alcohol-related behaviors significantly differed as children transitioned from elementary, middle, and high school. The alcohol-related problems included (1) progressing from a nonuser to a current user during the transition from elementary to middle school, (2) continued drinking in middle school, and (3) heavier more episodic drinking in high school. It is this transitional period that could use considerable more attention. 


\section{Physical Activity: Operationalization, Inactivity Patterns, and Research Links to}

\section{Adolescent Alcohol Use}

To date, research on adolescent alcohol use has focused on identifying risk and protective factors, studying different populations (e.g., various ethnic groups), and testing a variety of measures and explanatory models. Recently, there has been growing interest in how physical activity may prevent or reduce alcohol-related risk behaviors among teenagers. According to Caspersen and colleagues (1985), physical activity is defined as "bodily movement produced by the contraction of skeletal muscles that increases energy expenditure above the basal level" (p. 126, Caspersen, Powell, \& Christenson, 1985). These activities can be categorized into sports, conditioning, occupational or other activities (Caspersen et al., 1985). Exercise, according to Caspersen and colleagues, is a "subset of physical activity that is planned, structured, and repetitive and purposeful in the sense that improvement or maintenance of physical fitness is the objective" (p. 128, Caspersen, Powell, \& Christenson, 1985). For example, most conditioning and many sporting activities are performed in an effort to improve or maintain physical fitness. In most instances these activities are planned, structured, and repetitive. Physical fitness includes, but is not limited to, cardiorespiratory endurance, muscle strength, body composition, and flexibility, compromising a set of attributes that people have or acquire that relates to their individual ability to perform physical activities (Capersen et al., 1985; Thompson et al., 2003).

Everyone performs physical activity in some form in order to sustain life. There are various different operationalizations and proxies of physical activity. However, for purposes of the proposed study, physical activity will involve typical proxies of exercise- 
related activities (e.g., running, aerobic exercise, strength conditioning, flexibility stretching, calisthenics), which are subcategories of physical activity. Classification of physical activity is usually based on intensity - the amount of energy or effort a person expends in performing the activity (USDHHS, 1996; Ainsworth et al. 2000). Measurements of physical activity (in the form of exercise) are multi-faceted: frequency, intensity, time period (or duration), and type (or mode) (Ainsworth et al., 2000). Classification of physical activity (e.g., exercise) is usually based on intensity-the amount of energy or effort a person expends in performing the activity (USDHHS, 1996; Ainsworth et al., 2000).

While there has been a growing interest on the influence of physical activity on adolescent alcohol use, according to Healthy People 2010 (USDHHS, 2000), there exists widespread concern about physical inactivity among adolescents. The Surgeon General's report (USDHHS, 1996) and Healthy People 2010 and 2020 (USDHHS, 2000, 2010) identify the necessity of physical activity discuss the importance of its health-related benefits. Although there exists distinct health-related benefits from physical activity participation, physical activity rates have continued to decline over the past two decades (National Center for Health Statistics, 2005; CDC, 2008; USDHHS, 2000, 2010) despite efforts aimed at increasing exercise among adolescents.

The decline in physical activity has led to an alarming health trend. Over the past 20 years there has been a rapid increase in the prevalence of obesity in the U.S. (National Center for Health Statistics, 2005; USDHHS, 2010). According to the Center for Disease Control, obesity is defined as "a medical condition in which excess body fat has accumulated to the extent that it may have an adverse effect on health, leading to reduced 
life expectancy and/or increased health problems" (CDC, 2008). The Body Mass Index (BMI) is a measurement that defines people as overweight by comparing an individual's height and weight. Between the years of 1999 to 2002, 16.1\% of adolescents ages 12 to 19 , were overweight (95th percentile BMI for age) and 30.9\% were overweight or at risk for becoming overweight ( $85^{\text {th }}$ percentile BMI for age) (Hedley et al., 2004). Schools play an integral role in providing physical activity to American children and youth (Pate et al., 2006). The enrollment of high school students in physical education classes dropped from $41.6 \%$ to $28.4 \%$ between 1991 and 2003 (CDC, 2004). Thus, despite the growing interest in physical activity research, the relationships between risk and protective factors related to physical inactivity have not been thoroughly investigated.

Even fewer studies have examined the relationship between one of the most prevalent risks, alcohol use and one of the most important protective factors against adverse health outcomes, physical activity. The little research done to date concerning the relationship between physical activity participation and alcohol use among adolescents has been inconsistent. Some studies suggest that physical activity participation in the form of organized sports puts youth at increased risk for alcohol use (Barber, Eccles, \& Stone, 2001; Darling, Caldwell, \& Smith, 2005) while other studies find that youth engaged in organized sports or in other fitness activities are at lower risk (Collingwood, Sunderlin, \& Thomas, 2000; Werch et al., 2003; Werch, Moore, DiClemente, Bledsoe, \& Jobli, 2005). As a result of these mixed findings, the nature and extent of relations among physical activity, specific risk or protective factors, and health risk behaviors like underage drinking remain pressing topics for empirical research. Furthermore, exploring the relationships among these variables, more specifically, during 
the middle phases of adolescence (13-15 years of age) in which adolescence face peer pressure to use alcohol and engage in other health risk behaviors, is critical and provides considerable support for this line of research.

Moreover, the inconsistent findings suggest that it is important to look beyond direct effect models of the relation between physical activity and adolescent alcohol use. Physical activity may affect adolescent alcohol use, not directly, but indirectly by reducing the influence on alcohol use by way of a third variable in a mediating relationship. The premise of this proposal is that the inconsistent research findings to date are the result of the lack of attention among the dominant conceptual models to the possibility of a mediated relationship between physical activity and adolescent alcohol use. Evaluating more complex models (e.g., mediation) is important if the nature of the relationship between physical activity and adolescent alcohol use is to be better understood.

\section{Depressive Symptomatology: A Mediation Approach}

Health behaviors of greatest medical, research, and policy concern today are extremely complex (Kraemer, Stice, Kazdin, Offord, \& Kupfer, 2001). So, an understanding of how potential risk and protective factors work together is crucial to the advancement in research, policy, and practice. A mediation framework was used in the conceptualization of the proposed study. However, a way to understand why mediation was selected is to take a closer look at two major effect variables. There are two effects that add complexity to revealing and evaluating change; those are moderators and mediators. 
A moderator is a variable that affects the magnitude or level of effect across another variable (Kraemer et al., 2001). A mediator occurs within a causal pathway from an independent to a dependent variable (Kraemer et al., 2001). Mediators identify possible mechanisms through which a treatment and/or intervention might achieve its effects. The mediation model provides a strong foundation for this investigation by attempting to demonstrate a causal relation and understanding of the mechanisms of action through which physical activity influences later alcohol use among adolescents.

One proposed candidate for mediation identified from the research literature is depressive symptoms. Within the context of the proposed study, depression meets some of the elemental conditions for a mediator for two important reasons. First, depression is correlated inversely and robustly with physical activity and sport participation (Sanders, Field, Diego, \& Kaplan, 2000; Gore, Farrelll, \& Gordon, 2001; Kirkcaldy, Shephard, \& Siefen; 2002; Motl, Birnbaum, Kubik, \& Dishman, 2004). Second, depression is also correlated positively \& robustly with adolescent alcohol use (Kumpulainen, 2000; Diego, Field, \& Sanders, 2003; Hallfors, Waller, Bauer, Ford, \& Halpern, 2005).

Moreover, among adolescents, depressive disorders are viewed as one of the most prevalent psychiatric disorder (Santos, Richards, \& Bleckley, 2007; Lewinsohn, Hops, Roberts, Seeley, \& Andrews, 1993). Depressive symptoms demonstrate a dramatic increase from childhood to adolescence, where rates approximate those found in adult populations. Depressive disorders are estimated to affect 8.5 percent of adolescents, the equivalent of one in every twelve (Costello, Erkanli, \& Angold, 2006). Prevalence rates are estimated to be as high as 19 percent (Lewinsohn et al. 1993), and increase to 25 percent by the end of adolescence among teens in the general population (Kessler, 
Avenevoli, \& Merikangas, 2001; Kessler \& Walters, 1998). In addition to increased prevalence rates of depression, the average age of onset has rapidly decreased. By the age of 14 roughly 9 percent of adolescents have experienced at least one episode of severe depression (Avenevoli, \& Merikangas, 2001; Avenevoli, Knight, Kessler, \& Merikangas, 2008).

Depression is often seen with other conditions and associated with a range of negative health-related behaviors and outcomes during adolescence. (Keenan-Miller, Hammen, \& Brennan, 2007). Particularly relevant to the proposed study is previous research on adolescents suggests that there is a positive association between depression symptoms and substance use, including alcohol use (Diego et al., 2003; Kumpulainen, 2000). There are discrepancies as to whether depressed persons use substances to cope or whether substance use actually causes depression; moreover, the relation between depressive symptomatology and alcohol use may be bi-directional. Nonetheless, research has been unequivocal in demonstrating that depressed youth are more likely to use alcohol and other drugs than are their non-depressed counterparts.

Psychosocial stressors unique to and characteristic of adolescence likely influence the increase in depression symptoms among teenagers. During adolescence, teenagers begin to transfer a lot of attention to the self. This shift in attention in combination with beginning high school, physical and physiological development, and evolving associations with same sex and opposite sex peers, may have an adverse effect on the psychological well-being of adolescents. Participating in physical activity has been shown to promote more positive mental and emotional states via psychosocial mechanisms such as offering a diversion from distressing thoughts (Craft, 2005), or 
enhancing the ability to socialize with others and encouraging strong social networks (Salmon, 2001). Understanding what factors may ameliorate or promote depression during adolescence is important, because these factors may also influence engagement in risk behaviors, such as alcohol use.

Despite the consistency of the findings for an inverse relationship between physical activity and depressive symptomatology, few longitudinal studies have been conducted. Moreover, there is limited research on the potential relationships between physical activity, depression, and alcohol use across time. Given the comorbidity of depressive symptomatology and alcohol use and the positive mental health benefits of physical activity found in the literature, understanding more about the relationships between these constructs could have enormous public health impact.

\section{The Current Study}

Numerous factors contribute to individual differences in alcohol use behaviors among adolescents. Such health behaviors, such as adolescent alcohol use, are typically quite complex and involve not one distinct cause but part of a sequence of risk factors. (Kraemer et al., 2001). Given the strong associations between (a) physical activity and depressive symptomatology and (b) alcohol use and depressive symptomatology, investigations into the influence of physical activity on alcohol use behaviors among adolescents through a putative mediator such as depression is a logical next step. A mediation model is the best approach for determining how the relationship between physical activity and alcohol use among adolescents may be influenced by depression. No research to date has tested a mediation model. Demonstrating mediation would move the field forward by suggesting what might be the process resulting from physical activity 
that leads to reduction in adolescent alcohol use. Such a model could help advance the literature by elucidating one underlying mechanism linking two variables (e.g. physical activity and alcohol use) (Baron \& Kenny, 1986).

The current study expanded upon prior research concerning the effects of physical activity participation on later adolescent alcohol use, as well as the putative mediating role of depressive symptomatology during a particular period of growth and change. The overall purpose of the study is to examine (a) the temporal influence of early physical activity on alcohol use (e.g., frequency of alcohol use and binge use of alcohol) during middle adolescence, and (b) whether depressive symptomatology mediates the hypothesized relationship between physical activity and adolescent alcohol use (see Conceptual Models Figures 1 and 2 for proposed model).

The significance and innovation of this study lies in the ability to examine the relationships among all three constructs across time with a nationally representative sample (Add Health). This will aid in producing results that have greater external validity than many previous studies. In addition, more detailed information regarding how physical activity influences adolescent alcohol use can lead to development of new prevention and intervention programs as well as enhancing existing programs that aim to prevent an array of negative health behaviors such as alcohol use. 


\section{CHAPTER II. REVIEW OF THE LITERATURE}

A significant amount of literature exists concerning adolescents, depression, physical activity, and alcohol use. However, there is a dearth of research regarding the intersection of these concepts in helping to explain the influence physical activity has on alcohol use. Moreover, the importance of exploring this line of research among those transitioning from middle school to high school is reviewed. In this chapter, summaries

of the constructs are presented in such a way as to explain the evolution of the goals of the current study. Next, the theoretical underpinnings for the current research are reviewed. Finally, based on the review of the literature, the hypotheses are outlined.

\section{Population of Focus: Adolescents}

Defining Adolescence. Adolescence is a distinctly compelling stage of development because of the variety of changes and transitions occurring. According to Adams (2000) and other researchers, the body, in many ways, undergoes a second and dramatic growth period; much "like that of infancy" (Adams, 2000, p.3; Zucker, 2008). The rate of developmental change that occurs during adolescence is second only to infancy (USDHHS, 2010). Adolescence incorporates the transition from childhood to adulthood and is marked by the dramatic physical growth and neurological development, as well as considerable amounts of social and emotional changes (e.g. peer and societal expectations and self-identity issues) that occur during this stage of life (Wagner, 2008; Windle et al., 2009). During this developmental period, three quite distinct influences, biological, psychological, and social influences, begin to converge into one dynamic influence on adolescent behavior (Adams, 2000; Zucker, 2008). In addition, adolescence is often described as a period of experimentation with risky or problematic behaviors 
(Terzian, Andrews, \& Moore, 2011). Those behaviors include drinking, using drugs, smoking cigarettes, having unsafe sex, and driving recklessly. These behaviors, in many instances, prevent some adolescents from reaching their full potential and more importantly can lead to seriously impaired lives and even premature deaths (Sunstein, 2008; Ozer \& Irwin, 2009). As a result, there is so much to observe, analyze, and understand that this particular period in development lends itself well to research.

Researching the exploration of risky behaviors during adolescence is imperative to gain the knowledge necessary to develop appropriate preventative services and policies to improve overall health and health outcomes of the adolescent population. The age ranges that encompass adolescence vary depending on the source. However, the majority define adolescence as capturing the period in life between 10 and 20 years of age (USDHHS, 2007; CDC, 2009a; World Health Organization [WHO], 2011). Furthermore, according to Harter \& Monsour (1992), the period of adolescence can be further subdivided into three distinctive phases: early adolescence (ages 11 to 14), middle adolescence (ages 15 to 17), and late adolescence (ages 18 to 20).

For both males and females, significant changes occur in the neurobiological, hormonal, psychological, and social systems during adolescence (Cameron, 2004; Nelson, Leibenluft, McClure, \& Pine, 2005, Masten, 2007). However, a key biological marker of adolescence is puberty. The external signs of reproductive development are brought on by the hormonal changes occurring during puberty in combination with the formation of new more defined neural circuitry and wiring in the brain (Sisk \& Zehr, 2005). This interaction between external and internal forces impacts how individuals respond to, interact with, and view their environment. Any time between the ages of 8 
and 14 puberty can commence. However, by the age of 10 the majority of adolescents are in the midst of it as indicated by developing changes in the secretion patterns of the sex glands for males and females (Sisk \& Zehr, 2005). The timing and pace of puberty varies across individuals (Sisk \& Zehr, 2005). In order to gauge when adolescence begins, researchers have examined the first signs of puberty. For girls, the first visible sign is the appearance of breasts, generally between 10 and 11 years of age. For boys, the first physical sign of puberty is an increase in testicular size, generally occurring between 9 $1 / 2$ and 13 1/2 years of age. These very distinct developmental processes that characterize this age range makes focusing on this particular period as it relates to alcohol use of critical importance.

Research over the last several decades examining potential risk factors for problematic alcohol use has pointed toward psychosocial factors that may interfere with development during childhood and adolescence. These developmental periods serve as foundations for cognitive, biological, emotional, and social determinants of risk to converge in ways that encourage or deter individuals from risky alcohol consumption (or other risky or reckless behaviors). Alcohol expectancies have been thought to be acquired early in life (Goldman, 1989, 1999) and can influence their intentions to drink in the future (Austin \& Meili, 1994), as well as, the amount of alcohol that they consume. As early as preschool, children have been shown to discriminate alcohol from other fluids, and can express awareness that adults drink alcohol rather than children (Noll, Zucker, \& Greenberg, 1990). Furthermore, it has been well-documented that both positive and negative alcohol expectancies increase across age groups, from as young as first grade up through the twelfth grade. As described in an article by Zucker and 
colleagues, children younger than 10 begin to recognize and form opinions regarding alcohol and the consequences of that use (Zucker, Donovan, Masten, Mattson, \& Moss, 2009).

Although, adolescent alcohol use is common, the risks associated with alcohol use during key periods of development are significant. Grant and Dawson (1997) found that individuals who begin drinking prior to age fifteen are at four times the risk of meeting criteria for alcohol dependence as compared to those who begin after the age of twenty. For the proposed study, a sample of adolescents between 13 and 15 years of age will be followed across the transition from middle school (Wave 1) to high school (Wave 2) (e.g., $8^{\text {th }}$ to $9^{\text {th }}$ grade).

\section{Transition from Middle School to High School: Middle Adolescence}

Some researchers have acquired a heightened interest in school transition research because these education shifts impact and interact with concurrent developmental transitions. School transitions, for example, from elementary school to middle school to high school are typically perceived to be normal life experiences. However, these school transitions can be quite disruptive for students across various developmental domains (Benner \& Graham, 2009). For example, the high school transition involves negotiating shifts in organizational structure. This includes larger student enrollments, more bureaucratic organizational structure, and, in some cases, less personal attention than that experienced in elementary or middle school. These transitions involving the introduction to "unfamiliar, more populated, and less intimate school settings potentially disrupts social relations and supports (Baker et al., 2001). This could result in an individual feeling less a part of their community and a detachment from the reality of his or her self 
or environment (Lee \& Smith, 2001). The susceptibility to these feelings students may experience during school transitions may result from the dramatic developmental changes as well as changes in their school environments that are unfolding as individuals mature from childhood to adolescence (USDHHS, 2009).

To date, most research examining school transitions has focused on the shift from elementary to middle school, due to the simultaneous onset of adolescence and the changes in school environments. From elementary to middle school, students move from smaller, more intimate elementary schools to larger, more interpersonal, and often more academically demanding middle schools (Aikins, Bierman, \& Parker, 2005; Rudolph; Lambert, Clark, \& Kurlkowsky, 2001). Overall, research on the entrance into high school is more limited, although studies have documented analogous social, emotional, and psychological challenges to those experienced across the middle school transition (Barbara \& Olsen, 2004; Roeser, Eccles, \& Freedman-Doan, 1999). These include such things as depressive symptomatology, declines in self-esteem, falling academic performance, as well as social disengagement.

Key behavioral differences in alcohol use patterns begin to emerge during middle adolescence. These alcohol use behaviors include the change from (1) a nonuser of alcohol to (2) an initiator but not currently using to (3) an initiator and current user to (4) current user with heavy episodic drinking (Guo et al., 2000). This is of particular concern because there is a dearth of research exploring the transition from middle school to high school. These behavioral manifestations during this time period could have important effects on the use of alcohol in high school. This particular study focuses on adolescents transitioning from middle school to high school. Particularly specific to substance use, 
most transition research has focused on the transition out of high school. However, the transition out of middle school into high school is just as critical. What is quite unique and lends support to focusing on middle adolescence is studies show that increases in initiation and use of alcohol are evident during this transitional period (Faden, 2006; SAMHSA, 2008; Johnston et al., 2008; Windle et al., 2009). Moreover, as will be discussed in the review of the literature, there is also a simultaneous decrease in physical activity during middle adolescence (Singh et al. 2006).

\section{Adolescents and Alcohol Use}

Underage alcohol use is quite widespread, as shown by national surveys (USDHHS, 2007; Johnston et al., 2008; SAMHSA, 2009). Almost 74\% of persons age twenty-one or older report that they started drinking alcohol before reaching the legal age (Newes-Adeyi, Chen, Williams, \& Faden, 2005). Although considered widespread, trend data on alcohol use and alcohol dependence and abuse among persons aged 12 to 20 from 2002 to 2007 have found that among both males and females, rates of lifetime alcohol use decreased between 2002 and 2006 (NSDUH, 2008). Rates of lifetime alcohol use decreased from 56.5 percent in 2002 to 54.0 percent in 2006 for underage males and from 56.0 to 53.7 percent for underage females (NSDUH, 2008). Among underage males, the prevalence of past year dependence or abuse was lower in 2006 (9.6 percent) than in 2004 (10.8 percent) or 2002 (10.9 percent) (NSDUH, 2008). Among underage females, the prevalence of past year alcohol use was lower in 2006 (46.2 percent) than in 2003 (48.0 percent) (NSDUH, 2008). There were no differences between 2006 and the previous 4 years of the survey for either males or females on past month alcohol use, binge use, or heavy use. Unfortunately, in terms of rates of dependence and abuse there 
are no more current data available as of 2010. Thus, it is difficult to assess whether these patterns have remained stable or are undergoing change.

Despite trend data suggesting these slight decreases in alcohol use among adolescents (SAMSHA, 2008, 2009), the rates of alcohol use remain at high enough levels to still be a considerable public health concern and problem in the United States (Windle et al., 2009). Moreover, there have been increasingly higher rates of alarming drinking behaviors such as binge drinking and daily drinking (Windle et al., 2009). A distinctive and normative characteristic of adolescence is the increased initiation and experimentation with alcohol (Windle et al., 2009). Further details on the magnitudes of prevalence rates and trends in adolescent alcohol use are described in the next section.

Prevalence of Adolescent Alcohol Use. On an average day, almost 8000 adolescents, aged 12 to 17 , drink alcohol for the first time (SAMHSA, 2008). The severity of the concern and the need for further research concerning adolescent alcohol use is best described and understood in terms of the actual number of adolescents who drink and their patterns of alcohol consumption. Approximately 11 million American youth under the age of 21 drink alcohol with people aged 12 to 20 years drinking roughly 11 percent of all alcohol consumed in the United States (SAMHSA, 2008). Compared to tobacco or illicit drugs, alcohol, is the number one substance of choice among America's adolescents (Johnston et al., 2008; USDHHS, 2007).

Research also suggests that a considerable number of adolescents start drinking at very young ages. In fact, the Leadership to Keep Children Alcohol Free initiative was launched by the National Institute on Alcohol Abuse and Alcoholism to inform the public about the prevalence and impact of early alcohol use by young persons between 9 and 15 
years of age (D'Amico \& McCarthy, 2006). Since 1965, the average age of first alcohol use has decreased, demonstrating that youth are beginning to drink at a younger age (SAMSHA, 2008). From a historical perspective, in 2003, the average age of first alcohol use was roughly 14 years of age, compared to about 171/2 in 1965 (Newes-Adeyi, G. et al., 2005). Current data from more recent surveys continues to demonstrate this trend. For many, initiation of use occurs prior to age 13 (National Center for Chronic Disease Prevention and Health Promotion, 2008; Komro, Tobler, Maldonado-Molina \& Perry, 2010), with one-quarter of adolescents beginning to drink alcohol before the age of 13 (National Center for Chronic Disease Prevention and Health Promotion, 2008).

Data from the Youth Risk Behavior Surveillance System (YRBS) further underscore why there should be concern about alcohol initiation among our youth population. According to this survey, roughly 30 percent of youth in a sample of $9^{\text {th }}$ through 12th graders reported starting to consume before the age of 13. This early initiation and heavy use of alcohol presents serious consequences for healthy development. For example alcohol regularly plays a role in a variety of unfavorable outcomes including academic failure (Grunbaum et al., 2004), engagement in other illicit drug and tobacco use (Grunbaum et al., 2004; Shiffman \& Balabanis, 1995), changes in the anatomy and operations of the developing brain (Crews, Braun, Hoplight, Switzer, \& Knapp, 2000; White \& Swartzwelder, 2005), are four times at risk of meeting criteria for alcohol dependence as compared to those who start drinking after age twenty (Grant \& Dawson, 1997), and death from injuries (Hingson \& Kenkel, 2004).

The percentage of adolescents who have tried alcohol greatly increases throughout the high school years (Johnston et al., 2008). Nearly three-fourths of 
adolescents $(72 \%)$ in 2009 had tried alcohol by $12^{\text {th }}$ grade, and $43 \%$ of $12^{\text {th }}$ grade students reported drinking within the last 30 days (Johnston et al., 2008), which indicates regular alcohol use. Particularly relevant to the proposed study is that although lifetime alcohol use and 30-day prevalence among middle and high school aged students declined overall from 1996-2006 as detailed above, middle school years $\left(7^{\text {th }}\right.$ and $8^{\text {th }}$ grade) remain the peak period for alcohol initiation. To illustrate, approximately $40 \%$ of $8^{\text {th }}$ grade have tried alcohol (lifetime use). In addition, $6 \%$ of $8^{\text {th }}$ graders in 2008 reported being drunk at least once during the past 30 days. By age 15, approximately 50 percent of adolescent males and females have consumed an entire drink of alcohol (USDHHS, 2007). According to data from the 2008 Monitoring the Future (MTF) study, approximately 18 percent of $8^{\text {th }}$ graders and 41.2 percent of $10^{\text {th }}$ graders have been drunk at least once (Johnston et al., 2008). By $12^{\text {th }}$ grade, $44 \%$ of students report past-month alcohol consumption, which is three times the percentage from $8^{\text {th }}$ grade.(Johnston et al., 2008).

Patterns of Drinking Among Adolescents. For the most part, society underestimates not only the number of adolescents who use alcohol (USDHHS, 2007), but also the amount of alcohol adolescents consume and the many risks and consequences that alcohol consumption creates for adolescent drinkers and nondrinkers as well as the population as a whole. In most survey reports estimates for the prevalence of alcohol use are reported primarily at three levels defined for both males and females and for all ages as (1) current (past month) use - defined as at least one drink in the past 30 days; (2) binge use - defined as five or more drinks on the same occasion (e.g., at the same time or within a couple of hours of each other) on at least 1 day in the past 30 days; 
(3) heavy use - defined as five or more drinks on the same occasion on each of 5 or more days in the past 30 days (USDHHS, 2007).

When youth drink, they tend to do so intensively, regularly drinking four to five drinks at one time (SAMSHA, 2008). Based on the 2006 National Household Survey on Drug Abuse, 91 percent of all drinks consumed by teenagers are consumed by those who drink heavily (Biglan et al., 2003; Snyder \& Sickmund, 2006; SAMSHA, 2007). Of the approximate 11 million American youth who consume alcohol, nearly 7.2 million are binge drinkers (individuals consuming five or more drinks in a single occasion) (SAMSHA, 2007). Although some recent national surveys have shown declines in drinking among both younger and older adolescents (Johnston et al., 2008); overall, there exists considerable overlap across major national surveys, indicating that alcohol use remains intertwined with lives of most adolescent males and females.

Variability in Adolescent Alcohol Consumption. Despite the fact that it is common for most U.S. youth to grow up in a culture infiltrated by alcohol, they are still not all at uniform risk for alcohol consumption or its possible consequences. Epidemiological data indicates that there are substantial individual level differences such as ethnic and gender differences in prevalence rates for adolescent alcohol use (Griffin et al., 2000; Blum et al., 2000; Reardon \& Buka, 2002; Flory et al., 2006). As reported in national surveys, there is a considerable amount of variation between Whites and other ethnic/racial minority groups with respect to alcohol consumption (National Institute on Alcohol Abuse and Alcoholism (NIAAA), 2005; SAMSHA, 2008). Minority youth typically begin drinking at older ages than their White non-Hispanic counterparts (NIAAA, 2005). In general, Whites drink more than other racial and ethnic minority 
groups. Furthermore, there is great deal of variability in consumption of alcohol across racial and ethnic groups.

Data consistently indicate that rates of drinking and alcohol-related problems are highest among white and American Indian or Alaska Native adolescents followed by Hispanic adolescents, African Americans, and Asians. More specifically, no matter the alcohol consumption pattern, African-American and Asian youth tend to drink the least, as shown in Figure 6 (USDHHS, 2007, NSDUH, 2008). When alcohol use is disaggregated by racial/ethnic groups, whites are more likely than other racial/ethnic groups to report current use of alcohol (56.2 percent). The rates were 43.3 percent for American Indians or Alaska Natives, 43.2 percent for Hispanics, 41.9 percent for African-American, and 37.0 percent for Asians (NSDUH, 2008).

In addition to racial and ethnic group difference, variability exists by gender. However, studies on overall gender differences in alcohol use during adolescence are not as straightforward. Historically over the last several decades, adolescent males were always found to consume more alcohol than adolescent females (NSDUH, 2008; Cheever \& Weiss, 2009). Some studies demonstrated a pattern similar to that illustrated among the adult population in that adolescent boys are more likely to engage in drinking behaviors than girls (Johnston, O’Malley, \& Bachman, 1985) and are more likely to exhibit problems and symptoms of AUD (Rohde et al., 2001). For example, males have been more involved in heavy drinking and drunk driving than females (Johnston et al., 2003). The National Household Survey on Drug Abuse (NHSDA, 2004) found, 36\% of adolescent males and $31 \%$ of females reported current alcohol use, $18 \%$ of males versus 
$11.5 \%$ of females reported binge drinking, and $23.6 \%$ of males versus $18.9 \%$ of females reported drunkenness.

Despite evidence from larger scale studies reporting a gender disparity, the gap is far less pronounced among adolescents than among adult populations. Furthermore, this gender gap, which appeared most notably among younger children, is closing. The prevalence rates of alcohol consumption for boys and girls between the ages of 12 and 14 and 15 and 17 did not deviate more than a few percentage points from the one another. More specifically, in all three racial groups (non-Hispanic white, African American, and Hispanic) are more inclined than boys to report having consumed alcohol in the past 30 days -9.8 percent of Hispanic females, 8.3 percent of non-Hispanic white females, and 4.8 percent of African-American females. These rates are comparable to 6.3 percent of Hispanic males, 7.5 percent of non-Hispanic white males, and 4.2 percent of AfricanAmerican males. Similarly African-American girls aged 15 to 17 also are more apt to drink higher amounts of alcohol than African-American boys of the same age (Hingson \& Kenkel, 2004). Other researchers suggest that the relationship between gender and drinking is contingent on age. Specifically, studies have reported a gender convergence in terms of ever drinking among older adolescents (Costa, Jessor, \& Turbin, 1999; White, Hill, \& Effendi, 2003), signifying that the age range of the samples studied may influence the assessment of gender differences. Overall, despite discrepancies, the results suggest that gender may have a considerable influence on depressive symptomatology and alcohol use outcomes.

Consequently, the study of alcohol use in adolescents is imperative given the widespread number of adolescents engaging in its use. Adolescent alcohol use impacts 
both genders and individuals from various ethnic backgrounds. Moreover, when individuals begin to drink before 15 years of age they are five times more probable of developing alcohol dependence or abuse later in life (Grant \& Dawson, 1997; NSDUH, 2004). Therefore, it is important to know and understand the prevalence and current trends concerning adolescent alcohol use at the earliest stages of one's life in order to prevent potential adverse social and health consequences. A review of these consequences are found below.

Consequences of Adolescent Alcohol Consumption. In addition to the high prevalence rates and the style of drinking, alcohol use among adolescents is associated with poor social and health outcomes (Shope, 2006; Johnston, O'Malley, Bachman, \& Schulenberg, 2005; Shrier \& Crosby, 2003; Brooks, Harris, Thrall \& Woods, 2002; Maney, Hingham-Grdill \& Mahoney, 2002; Poulin \& Graham, 2001; Cooper \& Orcutt, 2000). For example, each year about 5,000 people under the age of 21 are involved in alcohol related fatalities involving underage drinking (USDHHS, 2007). Roughly, 1900 (38\%) of the 5,000 deaths involve motor vehicle crashes, about $1600(32 \%)$ result from homicides, and about $300(6 \%)$ result from suicides (CDC, 2004; Hingson \& Kenkel, 2004; National Highway Traffic Safety Administration [NHSTA], 2003). Underage drinking is also affiliated with illicit drug and tobacco use, academic failure, and a host of other harmful physical effects, ranging hangovers to alcohol poisoning (Grunbaum, 2004).

Research on the long-term consequences of adolescent drinking is developmentally oriented and involves the continuity of alcohol use into adulthood. Many researchers assert that adolescent alcohol use merits scientific attention because a 
considerable proportion of underage drinkers drink heavily and experience physiological, social, and psychological consequences that interfere with development (Maney et al., 2002). For example, adolescents are four times more likely to experience motor vehicle crashes (CDC, 2009b) and unintentional injuries, such as suicide attempts and interpersonal violence after consuming alcohol (Shope, 2006; Miller, Naimi, Brewer, \& Jones, 2007). Specifically, the consequences of alcohol consumption for individuals who do not begin drinking until 21 compared to those who begin drinking before the age of 15 are staggering. For example, youth who begin drinking before the age of 15 are 12 times more likely to experience unintentionally injuries while under the influence of alcohol, 7 times more likely to be involved in a motor vehicle crash after consuming alcohol, and 10 times more likely to have been involved in a physical altercation of some kind after drinking (National Research Council and Institute of Medicine, 2004).

Furthermore, adolescents are less likely to use safe sex practices than nondrinkers, which results in unprotected sex, unplanned pregnancies, as well as multiple sex partners (Shrier \& Crosby, 2003; Maney et al., 2002) than those who do not consume alcohol. Moreover, those who consumed alcohol before 13 years of age had were twice as likely to have unintended sex and more than twice as likely to have unprotected sex (Hingson \& Kenkel, 2004). Thus, with these types of implications for youth who initiate and continue drinking alcohol, this proposed study attempts to investigate what behaviors (e.g., physical activity participation) and developmental mediators (e.g., depressive symptomatology) can influence adolescent alcohol use. 


\section{Various Indicators in Adolescent Alcohol Use and Alcohol Use Vulnerability}

The inconsistencies in the literature concerning the relationship between physical activity and alcohol use may be at least partly attributable to a lack of a standardized and developmentally appropriate measurements for adolescent alcohol use. The most commonly used outcome variables in adolescent alcohol treatment studies are quantity and frequency. However, experts in adolescent alcohol use problems differ on developmentally appropriate measurement of alcohol use outcomes (Wagner, 2008). Predictors and outcomes of alcohol use (e.g., quantity, frequency, binge, and problem drinking) described by Schulenberg and Maggs (2001) were found to co-vary positively within and across time, which suggests a simultaneous reinforcing influence among these variables (Wagner, 2008). In a study performed by Hays and Ellickson (1996), experts on adolescent and adult alcohol use were asked to provide opinions about different indicators of alcohol use. Results demonstrated considerable variability in opinions. The findings from Hays and Ellickson revealed that estimating adolescent alcohol misuse can be quite challenging and not an accurate science. In fact, there exists considerable disagreement even among experts about what constitutes developmentally appropriate alcohol use outcome measurements. Remarkably, the greatest disagreement was for quantity-frequency indicators. In particular, these types of indicators represent the primary outcomes reported in adolescent alcohol treatment studies.

While the predominant outcomes reported in the adolescent alcohol use literature have been quantity and frequency indicators, other outcomes have also been used, including (1) indicators of early onset and/or intentions of alcohol use, (2) specific high risk drinking behaviors (e.g., binge drinking), and (3) problem drinking or health risk 
behaviors associated with alcohol misuse (e.g., motor vehicle accidents, other drug use, or unprotected sex). Although each of these indicators appear to have face validity and are likely significantly and positively correlated with other indicators there still exists a great deal of controversy over which of these indicators is a developmentally appropriate representation of adolescent alcohol use problems. Most of the conventional outcomes used in adolescent alcohol treatment research are based off of those used in alcohol adult alcohol treatment research. The only exception is seen when measuring negative consequences of drinking; such as "trouble at school" or "made excuses to your parents about your alcohol use" (Wagner, 2008). The divergence in expert opinions has led to the use of multiple indicators to assess adolescent alcohol use. This being the case, it is imperative to closely examine the fit of the proposed model across the various measures in order to research and provide a more comprehensive understand of the relationship among the variables.

The findings related to the variability concerning the appropriateness of various adolescent alcohol use indicators underscore the importance of assessing the fit of the proposed model across various indicators of adolescent alcohol use. The proposed study will attempt to assess the variability in the fit of the model according to four distinct definitions of alcohol use (quantity, frequency, binge, and problem drinking). Furthermore, since the proposed study targets a young adolescent group, the fit of the model will also be assessed according to measures of early onset and initiation of alcohol use. 


\section{Depression during Adolescence}

Considered by some to be one of the most serious forms of childhood psychopathology, the emergence of depressive symptoms is another prevalent health issue among adolescents, and an additional focus of the current study. Depression during adolescence is particularly problematic due to the prevalence, chronicity, co-morbidity, and the pervasive consequences associated with depressive disorders (Cicchetti \& Toth, 1998; Kaufman, Martin, King, \& Charney, 2001; Miller, 2007). For many years research on adolescent depression was neglected. Studies of depression in adolescents did not begin until the late 1970's and the early 1980's (Kandel \& Davies, 1982). The reason for this was in large part due to the conceptualization of adolescence as being a period of such storm and stress that to actually investigate adolescent depression appeared to be complex and daunting. This created a misconception that depression was a part of normal development. In addition, psychoanalytic theory, which was heavily influential for many years, thought it developmentally impossible for children and adolescents to become depressed (Rochlin, 1959; Rie, 1966; Hammen \& Rudolph, 1996; Son \& Kirchner, 2000). Furthermore, there was a belief that adolescents would eventually 'grow out' of depression (Petersen et al., 1993). Prior to this was the belief that depression during adolescence was not real, masked, or symptoms were concealed and expressed in ways that were quite unlike those seen in adults with depressive symptomatology (Reynolds, 1985).

More recently, because of the importance of healthy development during adolescence there is a distinct interest in understanding the mental health status of this significant subpopulation in the United States (Keyes, 2006). Furthermore, the first onset 
of depressive disorders now take place earlier in life than in the past (Cross-National Collaborative Group, 1992). Clinicians have found that children as young as two years of age show symptoms of depression, such as guilt, self-hate, and sadness (Puig-Antich, 1982; Luby, Si, Belden, Tandon \& Spitznagel, 2009). Thus, with such a shift in perspective over the past forty years, childhood and adolescent depression has emerged as an issue at the forefront of developmental psychopathology, psychology, and other public health related disciplines. Adolescence, in particular, is a critical developmental period to investigate because of the many significant changes in depressive problems that occur during these fundamental years (Petersen, Sarigiani, \& Kennedy, 1991).

Prevalence of Depression among Adolescents. Although depression may originate prior to adolescence, reported prevalence estimates suggest that a considerable number of individuals experience significant emotional distress during the teenage years. Furthermore, virtually all studies reporting rates of depressive symptoms and diagnosed depression in community samples have concluded that the rates are much higher among adolescents than children. For example, Kashani, Rosenberg, and Reid's (1989) study of 8,12 , and 17 year olds in the community found the prevalence rates of depression rises four-fold from the age of 8 to the age of 17. Similar reports from the Isle of Wight study also indicate a three-fold increase in rate of diagnosed depression from age 10 to age 1415 (Rutter, 1989). More recent research has also found depression among adolescence, compared to other stages of development, to be significantly more prevalent (Calles, 2007; Avenevoli, Knight, Kesller, \& Merikangas, 2008; Lenz, Coderre, \& Watanabe, 2009). 
The prevalence rates of adolescent depressive disorders ranges from $1.5 \%$ to $8 \%$ in community and clinical samples, and lifetime prevalence through adolescence is estimated as high as 25\% (Kessler, Avenevoli, \& Merikangas, 2001; Kerig \& Wenar, 2006; Seeley, Stice, \& Rohde, 2009; NSDUH, 2011). A closer examination of prevalence rates reveals that clinical depression in adolescence has ranged from $0.4 \%$ to $8.3 \%$ (Birmaher et al., 1996; NSDUH, 2011); the first depressive episode among depressed adolescent cases is generally in late childhood or early adolescence, with a mean age of onset of about 15 years (Kessler et al., 2005); 20-40\% of adolescents report experiencing significant depressed mood (Petersen et al., 1993); 2-3\% of this population meets diagnostic criteria for depressive disorders (Petersen et al., 1993); and around 18-20\% of school populations have clinical levels of depressive symptoms (Reynolds, 1992; Marcotte, Fortin, Potvin, \& Papillon, 2002).

As a result of various sampling, diagnostic and analytic methods, studies have revealed different overall prevalence rates of depression in adolescents. For example, in a more recent study of young adolescents (12 and 13 years old), Kubik, Lytle, Birnbaum, Murray, and Perry (2003) reported a prevalence rate of $35 \%$ for elevated depressive symptoms (indicated by scores of 16 or higher on the 20-item Center for Epidemiologic Studies Depression Scale [CES-D; Radloff, 1977]). However, these estimates uniformly indicate that depression is a significant and serious condition among American youth.

Prevalence estimates suggest that a considerable number of individuals experience significant emotional distress during the teenage years (Rushton, Forcier, \& Schectman, 2002; Kessler et al., 2005; Knopf, Park, \& Mulye, 2008). Furthermore, epidemiological evidence suggests significant short-term and long-term ramifications of 
early experiences of mood disorders on future health and well-being. For example, Birmaher et al., (1996) reported that, among the youngest participants in the National Comorbidity Survey (NCS), the average depressive episode length among adolescents was seven to nine months, with a probability of recurrence of $40 \%$ within two years and $70 \%$ within five years. Prospective data show that adolescent depression in the general population is not time-limited but persistent and stable, at least through the transition to early adulthood (Birmaher et al., 1996; Kessler, et al., 2001; Lewinsohn, Rhode, Seeley, Klein, \& Gotlib, 2000; Lewinsohn, Rohde, Klein, \& Seeley, 1999).

Variability in Adolescent Depression. There are two distinctive features of depression during adolescence. One feature, discussed in the preceding section, is the substantial increase in rates of both depressive symptoms and diagnosable disorders. The second distinctive feature is that there are distinct sex and racial/ethnic group differences emerging. There exist gender differences, with females more likely to exhibit depressive symptomatology than males (Birmaher et al., 1996; Kessler et al., 1996; Essau, Conradt, \& Peterman, 2000; Hankin \& Abramson, 2001; NSDUH, 2011). The timing of gender differences remains relatively unclear. However, by age 15 the gender differences in depression is at the adult rate of about 2:1 (girls to boys) (Leiman \& Scott-Collins, 1999). For example, in Essau et al (2000), no significant differences between females and males could be found before age 14. However, after age 14, girls had a fixed and undeviating increase in depression compared to boys. The gender differences that emerge by age 1415 years appears to persist to adulthood, as one out of every four girls is likely to have experience with moderate to severe symptoms of depression (Leiman \& Scott-Collins, 1999). More recently, the National Survey on Drug Use and Health (NSDUH, 
2008)combined data from 2004 to 2006 to find that female adolescents were more than twice as likely to have experienced a major depressive episode in the past year as male adolescents (12.7 vs. 4.6 percent).

Many of the explanations of this difference implicate experiences from adolescence, such as pubertal status. More specifically, boys and girls undergo differences in biological maturation. For example, girls tend to place increased importance on physical appearance, interpersonal factors such as relationships, and as a result experience an increased risk for stress-inducing negative life events (Cyranowski, Frank, Young, \& Shear, 2000; Siegel, 2002). Given these findings it is important to study the vulnerability factors that may affect the current study model.

There has been less research on racial/ethnic differences in the prevalence of depression among adolescents and the work that has been done has yielded inconsistent findings. Some research supports an increase in depressive symptoms among Hispanic youth, as compared with European American and African American youth (Twenge \& Nolen-Hoeksema, 2002). However, other studies have compared racial or ethnic groups and have found no significant differences in the prevalence of depressive disorders between European American, African American, Hispanic (Angold et al., 2002; Costello et al., 1997) or American Indian youth (Costello, Farmer, Angold, Burns, \& Erkanli, 1997). Interestingly the small sample size of ethnic minority youth in most community studies of adolescents diminishes the statistical power to test differences in the prevalence of depressive symptomatology between specific ethnic subgroups (Abela \& Hankin, 2008). 
With increasingly more research interests being placed on racial/ethnic differences among adolescent psychopathology, more studies are beginning to surface were significant racial/ethnic group differences do exists (McLaughlin, Hilt, \& NolenHoeksema, 2007). For example numerous studies have reported differences in depression between Black and White adolescents (Franko et al., 2005; Kistner, David, \& White, 2003). In addition, some studies have found higher levels of depressive symptomatology among Hispanic adolescents than other racial/ethnic groups (Doi, Roberts, Takeuchi, \& Suzuki, 2001). Despite the literature being inconsistent, higher levels of depressive symptomatology have been consistently found among Hispanic adolescents; specifically females. Reasons for this finding include but are not limited to the conflicting gender roles established by the more traditional conventional Hispanic culture (Steidel \& Contreras, 2003).

Consequences of Depression. In addition to a risk for recurrence of depression, adolescents with depression are more likely to experience a number of negative outcomes across the lifespan. Some of the consequences of adolescent depression include adverse effects on growth and development, impairment of school performance and peer/family relationships (Birmaher et al., 1996; Kessler \& Walters, 1998; Bhatia \& Bhatia, 2007), physical health problems, increased risk of early pregnancy among girls (Birmaher et al., 1996; Keenan-Miller, Hammen, \& Brennan, 2007), and increased suicidality, including both suicidal ideation and attempts (Hacker, Suglia, Fried, Rappaport, \& Cabral, 2006).

Specifically, depression can lead adolescents to be absent from school or fall behind in school. Education is a critical foundation for future adult earnings. It is possible if attendance and performance in school are substantially altered by depression, 
adolescents may lose potential earnings in the future. Depression during adolescence is also associated with impairments in relationships with family and friends and in cognitive functioning (Kessler \& Walters, 1998). Moreover, recurrent problems that have been linked to adolescent depression includes the positive association depression has with risk behaviors such as substance abuse problems (e.g., drinking behaviors) experienced during adolescence and adulthood (Birmaher et al., 1996; Franko et al., 2005; Schepis, Adinoff, \& Rao, 2008), and is hence a focus of the current study. Given the link between depression, physical activity (e.g., positive health behavior), and alcohol use behaviors (e.g., negative health behavior) makes the need for further understanding of the effects of depression in relation to health behaviors clearly important.

\section{Depression and Alcohol Use during Adolescence}

The current study derives a portion of its significance from the emergence of depressive symptoms and alcohol use as prevalent health problems during the adolescent years. The frequent co-variation between symptoms of depression and alcohol use is well documented in the literature. Alcohol use and depressive symptomatology quite frequently co-occur and emerge as substantial mental health problems early in the life course when experimentation, regular use of alcohol, and the experience of alcoholrelated problems are typically initiated (Diego et al., 2003; Ozer et al., 2009; NSDUH, 2011). Although the temporal sequence of alcohol use and depression is difficult to determine, the observed associations between alcohol abuse and depression are well known.

Empirical evidence has demonstrated that comorbidity is a typical feature of both depression and alcohol use during adolescence. For instance, as many as $80 \%$ of 
adolescents with alcohol use disorders (AUD) are comorbid with at least one other psychiatric disorder, with affective disorders being among the most common (Clark \& Bukstein, 1998; Bellis, Narasimhan, Thatcher, Keshavan, Soloff, \& Duncan, 2005). A report based on the National Household Survey on Drug Abuse (NHSDA) suggests that the relationship between substance abuse and other mental health outcomes is considerable among the general population of American teens (SAMSHA, 1999). In particular, the results from this survey indicate that adolescents reporting serious emotional problems (including depressive symptoms) were twice as likely to use alcohol in past months compared to those with few problems and approximately four times as likely to be alcohol dependent. Moreover, severity of emotional problems was associated with an increased likelihood of each of the indicators of alcohol use employed by the survey: general use, binge drinking in the past month, drunkenness in the past year, and alcohol dependence. In more recent research similar findings have been found (Knopf, Park, \& Mulye, 2008; Ozer et al., 2009; NSDUH, 2011).

Additional adolescent studies have found similar results. Costello et al. (1999) used data from the Great Smokey Mountains Study (GSMS) to assess gender differences in pathways to substance use, and found that depression is associated with higher rates and earlier onset of substance use for both males and females (Costello et al., 1999). This finding is supported by evidence from the Methodology for Epidemiology of Mental Disorders in Children and Adolescents Study (MECA) (Kandel et al., 1997), which documented extremely high prevalence of other psychiatric disorders among two-thirds of the adolescents in the sample classified as weekly drinkers. Furthermore, Gorman \& Derzon (2002) reports that negative affect, particularly a proneness to experiencing 
depressed mood, is linked to drug and alcohol use in teens, suggesting that susceptibility to distress predisposes certain teens to drink (Gorman \& Derzon, 2002). Support for this view is not unanimous, however, as other researchers argue that the relationship between emotionality and alcohol problems has been overstated (Barnow, Schuckit, Lucht, John, \& Freyberger, 2002).

The temporal ordering of conditions is a common characteristic of comorbidity studies, as it can help establish the possible causal connection between co-occurring psychiatric disorders. Differences in sample selection, data collection, and periods of observation have led researchers to arrive at different conclusions about the association between depression and alcohol use (Angold, Costello, \& Erkanli, 1999). Despite the variability in the conceptualizing and researching of comorbid conditions, investigation into the co-occurrence of symptoms of depression and drinking during adolescence is imperative in attempting to address the complex nature of health behaviors and outcomes. What can clearly be seen from the studies mentioned above is that there is a strong linkage between depression and alcohol; what demands further research is the nature of the relationships between them. Furthermore, adolescence appears to be a crucial stage in the life course trajectory during which these disorders develop and begin to interact. The proposed study will pay close attention to the temporal ordering of physical activity, depressive symptomatology, and alcohol use to help resolve alternative and confounding explanations.

\section{Linking Patterns of Physical Activity Participation to Alcohol Use}

Although research estimates that $25 \%$ of an adolescents time is spent engaging in school and academic work (Larson. 2000), it is clear that the other remaining time is 
spent engaging in many other activities that are likely to have impact on them as individuals. How adolescents spend their leisure time is important to understanding the complex interactions they have on a daily basis. When asked to self-report their feelings about activities throughout the day, adolescents often report high levels of boredom and low levels of involvement in challenging activities (Larson, 2000; Larson \& Richards, 1991). As a result, researchers are beginning to turn their attention to exploring how involvement in various forms of physical activity and exercise involvement can influence health outcomes (e.g. alcohol use) among adolescents. There is compelling evidence that adolescents who are more involved in extracurricular activities (e.g., physical activities) experience better emotional and social outcomes, including improved psychological wellbeing (Bohnert \& Garber, 2007; Larson, Hansen, \& Moneta, 2006).

\section{Extracurricular Activities: Advantageous to Youth Development}

Theoretically, general involvement in extracurricular activities (e.g., academic focused, interest focused, or athletic focused), done during or after school, should benefit adolescents because activities are often organized in ways that promote growth, prosocial opportunities and involvement, structure, and intrinsic motivation (Larson, 2000; Roth \& Brooks-Gunn, 2003). Research has provided evidence that involvement in extracurricular activities can be quite beneficial to the development of adolescents. Extracurricular activities, not only, allow for the development of areas of individual interest and talent, but also provide unique avenues for meeting new people with shared interests.

Academic benefits have also been seen in individuals involved in extracurricular activities. Compared to adolescents who do not participate in extracurricular activities, 
adolescents who participate in extracurricular activities have more positive outlooks on school, receive higher grades, and have higher academic goals (Darling et al., 2005). Extracurricular activities have also been associated with behavioral functioning. Mahoney (2000) conducted a longitudinal study examining the associations between patterns of antisocial behavior and extracurricular activities. The study found that the more involved an individual was in extracurricular activities the less likely they were to drop out of high school and be arrested in young adulthood.

Furthermore, psychological benefits associated with participation in extracurricular activities have been linked to higher levels of self-esteem, even several years after high school (Barber et al., 2001). Research has also shown a lower level of depressed affect in individuals involved in extracurricular activities (Mahoney, Schweder, \& Stattin, 2002). Therefore, there exists convincing evidence that participation in extracurricular activities is associated with positive psychological functioning. A limitation to studies performed to date is that extracurricular participation was considered only at one point in time. There is limited research exploring the continuity and predictive associations of extracurricular activities over time. Furthermore, studies have not examined relations between extracurricular participation and high-risk behaviors such as alcohol use. The present study will focus on the relationship of physical activity participation, a form of extracurricular activity, on depressive symptomatology and adolescent alcohol use.

\section{Adolescents and Physical Activity}

Because of its benefits, physical activity is number one on the list of the nation's leading health indicators, according to Healthy People 2010, a national health promotion 
and disease prevention program that sets a series of health goals by year 2010 (U.S. Department of Health and Human Service, 2000). The Surgeon General's Report (U.S. Department of Health and Human Services (USDHHS), 1996) and Healthy People 2010 clearly identify and describe the necessity of physical activity and the importance of its health-related benefits. The risk of many severe health problems, such as obesity and heart disease can be significantly reduced through involvement in physical activities.

However, many American adolescents are not meeting the recommended levels of physical activity. Nearly $80 \%$ of all children and adolescents do not meet the daily recommendations for physical activity set forth by the Department of Health and Human Services (DHHS) and the American College of Sports Medicine (2002) (Eaton et al., 2006). Both organizations recommend 30 minutes of exercise, five days per week. Despite these recommendations and the benefits, physical activity levels decline during adolescence (Telama \& Yang, 2000; Kimm et al., 2002; Gordon-Larsen, Nelson, \& Popkin, 2004).

According to the 2005 Youth Risk Behavior Survey (YRBS) data, 64.2\% of adolescents, in grades 9-12, participated in insufficient amounts of physical activity. Nearly one-third of students nationally $(31.2 \%)$ had an insufficient amount of physical activity, defined as not participating in vigorous physical activity for 20 or more minutes on three or more of the past seven days, nor participating in moderate physical activity for 30 or more minutes on three or more of the past seven days of the week (Grunbaum et al., 2002). Therefore as fewer adolescents participate in physical activity across adolescence, both patterns of physical activity participation and the relationships of these 
patterns to important developmental and health outcomes are likely to differ according to the period in the life span.

\section{Physical Activity and Depression}

The association between exercise related behavior and mental health has been examined by many researchers (Byrne \& Byrne, 1993; Gauvin \& Spence, 1996; North et al., 1990; Salmon, 2001). Since 1984, over 1,000 studies have been conducted examining the relationship between physical fitness and mental health, with the majority focusing on the therapeutic effects of physical activity in the reduction of depression (Phillips, Kiernan, \& King, 2003). After controlling for social class and health status, one population based study reported higher emotional well-being among physically active youth (Steptoe \& Butler, 1996). Research in this area has been plagued with methodological limitations, such as small sample sizes, and lack of control groups and random assignment.

In addition, cross-sectional studies along with two large population studies (Farmer et al., 1988; Weyerer, 1992) have found that regular exercise is inversely related to indicators of mental illness, such as depression and depressed mood, among both adults and children. Evidence suggests that if the incidence of depression among adolescents can be reduced it is more possible to protect adolescents from maladaptive behaviors such as substance abuse (Kirkcaldy et al., 2002; Nelson \& Gordon-Larsen, 2006; Eaton et al., 2006), and increasing physical activity may be an especially reliable strategy for reducing depression. Despite the existence of this literature and the growing interest in physical activity research, the relationship between risk and protective factors and physical activity has not been thoroughly investigated. Furthermore, even fewer 
studies have examined physical activity as a protective factor for risky health behaviors such as alcohol use (Arthur et al., 2002; Jessor, 1991; Nelson \& Gordon-Larsen, 2006; Pate, Heath, Dowda, \& Trost, 1996; Werch et al., 2003).

\section{Physical Activity Participation and Alcohol Use}

Physical activity participation was chosen as the focus of the current study for several reasons. First, the health benefits of regular physical activity are well established. Second, on average adolescents spend the majority of their leisure time in athletic activities (Larson \& Seepersad, 2003; Linver, Roth, \& Brooks-Gunn, 2009). Third, the outcomes of sports participation, especially in terms of substance use, have been the least consistent compared to other extracurricular activities (Eccles \& Barber, 1999; Fredricks \& Eccles, 2006).

Recent research has examined correlations between alcohol use and physical activity. Research has found that as age increases, alcohol use increases, and physical activity declines (Singh, Mahashwari, Sharma, \& Anand, 2006). The National Growth and Health Studies found a $34 \%$ decline in physical activity in youth during this transitional period from 10-18 years of age. Data from the YRBS found that vigorous physical activity declines about $30 \%$ between grades 9 and 12 . Thus, the decline in physical activity begins early, about the time of entry into middle school, and continues throughout adolescence. It is a widely held perception that physical activity behavior is linked to other positive health behaviors among teenagers. In contrast, unhealthy behaviors tend to cluster together.

As mentioned earlier, alcohol is the drug of choice for youth, with approximately 11 million American youth under the age of 21 consuming alcohol. The peak years of 
alcohol initiation are $7^{\text {th }}$ and $8^{\text {th }}$ grades (Faden, 2006) with the prevalence of consumption rising steeply during adolescence until it plateaus at about age 21 (USDHHS, 2007). Individuals who use alcohol usually also smoke, are physically inactive, and eat fewer fruits and vegetables (Poortinga, 2007; Thatcher \& Clark, 2006). A commonly held view is that time spent engaged in physical activity is time that is not available for negative health behaviors such as substance abuse (Pate et al., 1996). This logic has led some researchers to recommend exercise to be incorporated into youth intervention/prevention programs to prevent an array of negative health behaviors such as alcohol use (Collingwood et al., 2000; Werch, 2003; Moore \& Werch, 2005; Matthews et al., 2007).

\section{Theoretical Perspective on Physical Activity Participation and Alcohol Use}

There are three common theoretical perspectives for the influence physical activity participation can have on psychological and behavioral outcomes. According to the Zero-Sum Model of sports participation, there is only a limited amount of time that an adolescent can devote to academic, social, and other leisure activities (e.g. Coleman, 1961). Therefore, it is possible that time spent in athletics could take away from time devoted to academics, potentially leading to declines in academic pursuits. Where this theoretical perspective could have an impact on alcohol use is in the idea that athletics may also shift and individual's attention to social and peer-oriented activities, which could influence behavioral outcomes such as substance use.

A second theoretical perspective which is a modification to the Zero-Sum Model, known as the Threshold Model (Marsh, 1991), states that participation in athletics to a certain extent can be advantageous, but extreme levels of participation are likely to take away from other areas and lead to possible maladaptive outcomes. In general, the Zero- 
both of these models suggest that forms of sports participation can be linked to academic difficulties which are linked to a higher likelihood of substance use. These perspectives would lean more toward the notion that participation in sports could be a risk factor during adolescence.

The third perspective is the Developmental Perspective, which claims that participation in sports promotes positive growth and development for adolescents and should not be viewed in competition with other domains of functioning (e.g., academics) (Holland \& Andre, 1987). Marsh (1993) proposed that athletic participation could be developmentally beneficial in many domains, including academics, by providing adolescents with a means through which they could more associated in prosocial capacities with their school bond and practice commitment to a particular activity. Research has shown that individuals who perceive themselves as "jocks" actually associated more with peers that valued education, suggesting that sports involvement could be associated with positive adaptive functioning in other domain such as academic pursuits (Barber, Eccles, and Stone, 2001). According to the Developmental Perspective, sports participation is viewed as a promotive factor, or a variable that predicts better developmental outcomes for all adolescents, regardless of risk level (e.g. Gutman, Sameroff, \& Cole, 2003). Therefore, sports participation could be seen as a resource upon which to gain resiliency despite risk.

The Zero-Sum and Threshold models view physical activity participation as a risk factor and the Developmental Perspective views physical activity participation as a protective factor. The different theoretical perspectives could explain the inconsistencies seen in the literature. The model underscored in the current study is the developmental 
perspective. Furthermore, the study will test whether in fact there is a positive influence of physical activity on alcohol use behaviors.

\section{Review of Studies on Physical Activity Participation and Alcohol Use: Variability in}

\section{Findings}

While studies have attempted to explain the relationship between alcohol and physical activity, findings are not consistent across studies. Moreover, few studies have examined the potential protective effect of physical activity on alcohol use. Some studies identify sports participation as a risk factor for substance use, whereas others suggest sports participation promotes less substance use among adolescents. The research literature is not only inconsistent, but there have been no studies to date that have examined the associations in a nationally representative sample of U.S. youth. Furthermore, potential moderators such as gender should be considered as levels and types of sports participation vary according to these variables. In addition to the reported inconsistencies, few studies have examined the potential influence physical activity has on depressive symptomatology to help explain the relationship between physical activity and alcohol use.

In many studies, athletic participation has been a risk factor associated with higher levels of drinking (Barber, Eccles, \& Stone, 2001). This finding has been seen even after demographic characteristics are controlled (Darling, Caldwell, \& Smith, 2005). One study that examined alcohol use and physical activity found that an increase in physical activity was positively correlated with an increase in alcohol use (Rockafellow \& Savies, 2006). In this study, college age persons who were extrinsically motivated to participate in exercise by encouragement from others, and involvement in organized sport 
teams were more likely to drink. Vickers and colleagues (2004) found similar results specific to binge drinking. Adolescents who reported binge-drinking episodes also reported more increased physical activity than those who were not binge drinkers (Vickers et al., 2004). The samples in both of these studies were college students, and findings may not be generalizable to aged and limits the generalizability to middle-school and high-school adolescents.

In younger groups, inconsistencies in results have also been found. When a group of high schoolers were divided and examined based on their level of sport involvement, athletes tended to participate in more drinking behavior (Rainey, Mckeown, Sargent, \& Valios, 1996). In addition, only two studies have examined physical activity as a protective factor for alcohol in adolescents. Project SPORT involved a brief intervention and wellness brochure given to adolescents specifically aimed at decreasing alcohol use while also increasing physical activity (Werch et al., 2003). The SPORT Plus intervention was found to have a positive effect for decreased alcohol consumption and increased physical activity participation. These results, however, were only present at three-months and six-months post-intervention, and no significant results were seen at the 12-month follow up. However, a second study found that highly active athletes had the highest rates of alcohol consumption when compared to low active athletes and nonathletes (Elder et al., 2000). Furthermore, no significant findings were observed when different levels of physical activity were examined.

Researchers have also identified gender as a factor that might account for the inconsistent findings surrounding the relationship between physical activity participation and alcohol use. However, even these results have conflicted with one another, with 
some studies linking sports and drinking among males only (Aaron et al., 1995; Eitle, Turner, \& Eitle, 2003; Jerry-Szpak \& Brown, 1994; Mays \& Thompson, 2009) and other studies suggesting that sports may be protective against drinking for males only (Fredricks \& Eccles, 2006). Finally, some researchers have pointed to gender by sport interactions, such that the sports associated with higher levels of drinking among males differ from the sports associated with higher levels of drinking among females (JerrySzpak \& Brown, 1994; Moore \& Werch, 2005; Ford, 2007). Specifically, for females school-sponsored dance, cheerleading, and gymnastics were associated with a decreased risk of alcohol use, whereas those in the same activities out of school were at increased risk for substance use. Moreover, for males, out of school swimming was associated with a decreased risk of heavy alcohol use, while in school involvement in football, swimming, wrestling, or tennis were associated with an increased risk for substance use. These inconsistencies demonstrate the importance of attending to gender when studying physical activity participation.

Changes in physical activity and alcohol use are two important issues in adolescence. However, conflicting findings in the extant literature suggest a complex relationship between physical activity and drinking behavior in adolescence. These results also demonstrate the need to explore potential developmental mediators that may be working simultaneously and that may explain the variability in the research findings. The findings suggest that perhaps additional variables are being overlooked in the relationship between physical activity and alcohol use. The majority of research has focused on the issues independent of one another. In addition, no studies to date have explored the relationship between physical activity and correlated risk factors for alcohol 
use, such as depression. The current study addresses the need for this line of inquiry by examining gender differences in depressive symptoms, alcohol use, physical activity participation, and the influence of physical activity participation on depressive symptoms and alcohol use.

Risky behaviors are considered to increase negative health-related outcomes for

all persons. Given the significant number of adolescents who engage in limited physical activity and partake in risky alcohol use behaviors, continued research on this population and the interaction of physical activity on depressive symptomatology and alcohol use behaviors is critically important. This chapter has highlighted the significance of adolescent risk behaviors and discussed potential interrelations among three variables (e.g., physical activity, depressive symptomatology, and alcohol use) during a critical time in human development. A unique feature of this study lies in the potential to expound upon these relationships with a national data set and further increase knowledge on the complexities of human behavior and resulting health outcomes.

\section{Theoretical Framework for Current Study}

When attempting to understand the experiences of adolescence, it is important to note that behavior is shaped, in large part, by both individual and environmental factors. It is the interplay of an individual's characteristics within their own environmental context that ultimately influences behavior and, in the long run, developmental outcomes (Jenson \& Fraser, 2006). This is why so many in social work and other helping professions have embraced ecological and system theories in order to understand human behavior. The understanding of how the individual and the environment influence one another is critical to understanding and approaching human behavioral change. However, 
understanding human behavior and health outcomes such as physical activity, depression and alcohol use requires more specific knowledge. This study seeks to integrate two seemingly disparate constructs within one overarching conceptual framework. In other words, this study seeks to understand adolescent alcohol use outcomes through an examination of differences in physical activity behaviors and depressive symptomatology.

The theoretical rationale for the proposed study is organized within a "risk and resilience" perspective of health behaviors and outcomes. Researchers examining the concept of resilience are typically invested in exploring, identifying, and understanding the vulnerability to adverse life circumstances and the protective factors they may modify negative life circumstances. The researchers then look for the possible mechanisms that might underlie these associations found (Luthar, Cicchetti, \& Becker, 2000). A particular aspect that has been emphasized in regards to the risk and resilience framework is the need to analyze components within an interactive perspective. Neither risk factors, nor protective factors act independently of one another and their effects are quite often associated (Rutter, 1990). Furthermore, depressive symptomatology and physical activity can be looked at in terms of risk and protective factors respectively for adolescent alcohol use outcomes.

In this particular study, the health outcome of interest is alcohol use and the risk and protective factors are depressive symptomatology and physical activity participation respectively. The mechanism by which to understand the relationship between physical activity and alcohol use has been identified in this study as depressive symptomatology. Therefore, each of the pivotal terms; risk and protective factors, and resilience are 
defined in the preceding section, as well as a review of the key variables in the proposed model and their ties to the risk and resilience framework.

\section{Risk and Resilience Perspective: Defining the Terms. From a historical} perspective, the study of the classic terms, risk and resilience have a foundation in the study of medicine. A longitudinal study of heart disease, known as the Framingham Study, performed in 1949 by Thomas R. Dawber and his colleagues followed participants over periods of time in order to understand how those who developed heart disease differed from those who did not (Dawber \& Kannel, 1966). One of the primary goals of the study was to predict those who were most likely to develop the disease, thereby proposing suggestions for prevention. One of the major contributions of the study lies in the institution of the modern use of the term risk factor, defined as a variable or attribute that increases the probability, digression, or maintenance of a particular outcome or condition (Jenson \& Fraser, 2006).

The value of this form of research became increasingly more pronounced and began to spread across disciplines and take on wide-range applicability and utility for research. In addition to identifying risk factors for certain conditions, risk and prevention researchers came across another concept. In 1955, Emmy Werner (1994) began another longitudinal study known as the Kauai study. This study followed individuals from before birth through adulthood living impoverished, stressful, and disruptive family settings. Werner identified these life circumstances as risk factors for a number of physical, psychological, and social difficulties. However, what was even more intriguing was that from adolescence into adulthood, $33 \%$ of those who were thought to be "high risk" actually became highly functioning, adjusted and capable individuals (Werner, 
1994). As a result of this research, it became clear that some people could survive and succeed regardless of being exposed to perceived risky circumstances. This finding marked the beginning of resilience research.

Resilience is defined as an active process wherein individuals display positive adaptation despite experiences of significant adversity or trauma (Luthar, Cichetti, \& Becker, 2000). Another definition that supports the proposed study is resilience defined as an adaptive behavior that produces positive social and health outcomes arising from the dynamic interaction between risk and protective factors (Rutter, 1987). The notion of resilience is a positive complement to the negative connotations of risk. However, the first step in resilience research is to identify the risk factors. Once identified, if certain individuals overcome these factors, the second step is to identify what about these persons have made them resilient. These findings have led researchers to become more interested in attributes that could protect at-risk individuals from the development of later difficulties and actually develop positive developmental outcomes (Rutter, 1987).

Protective factors are defined as attributes and resources that interact with stressors such that those who are appear to be in the presence of stressors are more likely to experience beneficial and buffering effects from psychological distress based on the presence of these resources (Masten, Best, \& Garmezy, 1990). Examples include an individual belief that you can control life events that could affect you (e.g., internal locus of control) or possessing a positive relationship with an adult figure in one's life. Individuals who are in the presence of significant life challenges and possess such positive attributes (e.g., positive factors) tend to fare than those who lack them. (Luthar \& Cicchetti, 2000). Conceptually, protective factors are perceived as individually distinct 
from risk factors and are often believed to modify risk. However, not in all cases are protective factors the opposite of or signify the absence of risk (Rutter, 1987).

For example, as Rutter explains from his research, a perceived positive marital relationship served as a protective effect for some; whereas for other individuals, it appeared just as likely to result in poor outcomes in the presence of a positive marital relationship. Therefore, it is not conclusive for one to think that in the presence of what presumes to be a protective factor for some that all individuals will respond in the same way resulting in the same outcomes. It is imperative to distinguish protective factors from promotive factors which exhibit a main effect on the capacity to protect all individuals the same way regardless of risk (e.g. Luthar,et al., 2000; Masten et al., 1990). This has widespread implications on designing effective intervention and prevention programs that identify and incorporate individually unique protective factors.

Resilience in Social Work Practice. It appears quite fitting that the concepts of risk and resilience made their way into social work practice; since the profession is known for studying and ameliorating human problems that involve several causes rather than a singular distinct cause. The identification of risk and protective factors offers an empirical pathway toward effective education, prevention, and other human services. The social work discipline now has a considerable number of researchers and practitioners interested in the resilience phenomena. More specifically, the interest is in how people have overcome challenging circumstances to lead healthy and successful lives, as well as in the growth of young persons, resource development, wellness, and health promotion (Benard, 2004). Resilience researchers who have invested time in exploring the development of youth into adults have documented invariable findings 
whereby the majority of youth, even those from highly stressed families or resource deprived communities somehow manage to become not only successful individuals but individuals with high levels of social, emotional, and intellectual strength (Werner \& Smith, 2001).

Among various youth populations, research has shown that a considerable number of youth at risk for future developmental problems become quite successful. In most studies, on average 70 to $75 \%$ of children in foster care (Festinger, 1984), from gangs, children from substance-abusing families (Werner \& Smith, 2001), children growing up in poverty, and even children who have been sexually abused (Wilkes, 2002) successfully navigate these challenging environmental circumstances. The above percentages provide convincing support for protective factors being more influential than risk factors in the developing lives and outcomes of children and their families (REF). The ability to overcome negative life circumstances is attributed to the discovery in resilience-based research that resilience is an individual ability and capacity for healthy growth and development even in the face of challenges (Benard, 2004).

Despite the positive connotations of "resiliency", the manner by which individuals are labeled as resilient perpetuates the illusion that resilience is something that one either possesses or does not possess. The illusion in turn creates the belief that if resilience resides within the individual, than those who are not resilient are somehow responsible for their own deficiencies. Furthermore, it is believed that nothing can be done around that individual to increase their chances of success. However, a lot of research provides evidence that not only the individual but parents, schools, and other 
social contexts are an important part of the development of "resiliency" (Werner \& Smith, 1992).

Risk and Protective Factors and Resilience in Adolescence. Adolescence is characterized by a rather extended transition phase in which the individuals is neither a child nor an adult (Cicchetti \& Toth, 2009). Adolescence is a period of identity formation and experimentation. A state of struggle between the adolescent and adult figures presents itself during adolescence, whereby the adolescent strives for independence, but parents and the rest of society still view the adolescent as lacking the skills necessary for full adult responsibilities. Consequently, creating potential internal and external conflict within the adolescent during this developmental period (Cicchetti \& Toth, 2009).

In an influential article, Arnett (1999) identified three central features of the stress that is experienced by some, but not all, adolescents as a result of this potential internal and external conflict: mood disruptions, conflict with parents, and engaging in risky behaviors. Research has shown that part of this developmental process includes the experimentation and development of risk and protective behaviors (Paglia \& Room, 1999; Arnett, 1992; Zoccolillo, Vitaro, \& Tremblay, 1999). The focus of the current study is on two such important behaviors, one risk and one protective, alcohol use and physical activity respectively. Moreover, the current study evaluates a specific risk factor for adolescent alcohol use (e.g., depressive symptomatology) and its relationship to a protective factor (e.g., physical activity) in order to explore the influence of depressive symptomatology as a potential mediator in the relationship between physical activity and adolescent alcohol use. Behavior and practices that begin in adolescence may carry over 
into adulthood and can have an impact on the development of an array of negative health outcomes.

Adolescence is so often associated with turbulent time, stress, intense moodiness, and preoccupation with the self. However, although adolescence can be a turbulent period, not all is grim during this critical developmental stage (Mulye et al., 2009). As previously discussed, some children who are at increased risk for health-compromising behaviors successfully navigate through adolescence, avoiding the acquisition of behaviors that predispose them to negative health outcomes; others, from more perceived positive environments develop a variety of health risk behaviors resulting in significant morbidity (Resnick et al., 1997). Adolescents possess a great deal of creative imagination, energy, and promise. Therefore, for some youth, adolescence can become a time to redefine themselves through involvement in new opportunities that enable them to recover or better adjust to change.

It is critical, as recognized by many developmental theorists, to study both abnormal and normal development in order to gain a more comprehensive understanding of human functioning and adjustment (Cicchetti, 1990). Some adolescents are resilient in the face of profound biological, psychological, social, and contextual (e.g., negative neighborhood, family and school environments) challenges occurring during this particular developmental period (Luthar, Cicchetti, \& Becker, 2000). These adolescents sometimes possess particular psychosocial resources, including but not limited to, better intellectual functioning, parents of good physical and mental health, parental availability, more positive self-concepts (Goldstein \& Brooks, 2006), effective schools, high neighborhood quality, and connections to caring adult mentors and pro-social peers 
(Wright \& Masten, 2006). Although adolescents exhibit wide variability, the fact that depressive symptomatology and increased alcohol initiation and consumption are not atypical during this period of development suggests that behaviors commonly associated with internalizing and externalizing forms of psychopathology may in fact be escalating (Cicchetti \& Toth, 2009). The remarkable changes that characterize adolescence serve as a powerful rationale for directing research efforts toward exploring the risk and protective factors that surround this population.

Current conceptualizations of adolescent behaviors are quite complex with a renewed value for the role of developmental processes in behavioral outcomes. Earlier work on adolescent risk behaviors was confined to a particular subset, often termed problem behaviors that involved such behaviors as alcohol abuse (Jessor, 1984). More recent research, however, has broaden the boundaries around the conceptual notion of problem behavior to include the functional commonality of these problem behaviors with other domains of adolescent behavior that also compromise development, including psychopathology (e.g. depression), as well as health-compromising behaviors, such as insufficient exercise (Jessor, 1984). The emphasis on depression and physical inactivity are, based on prevalence data for both conditions, becoming increasingly more common among adolescents creating a serious public health problem.

At the same time that there has been considerable advancement in identifying factors contributing to adolescent health risk behaviors, it has also become quite clear that the presence of risk factors alone yield a less than comprehensive account of involvement in risk behaviors. There exists great heterogeneity and variability in the likelihood of involvement in risk behavior among adolescents. This has created an important area of 
research on adolescent risk behavior, namely the identification and assessment of protective factors (Jessor, Turbin, \& Costa, 1998; Fergus \& Zimmerman, 2005). As such, research has begun to move to a resilience-oriented focus on protective factors (Veselska, Geckova, Orosova, Gajdosova, van Dijk, \& Reijneveld, 2009; Wille, Bettge, \& Ravens-Sieberer, 2008; Goldstein \& Brooks, 2006; Gutman, Sameroff, \& Cole, 2003; Jessor et al., 1995). As mentioned earlier, examples of protective factors include positive family characteristics (i.e. positive sibling relationships, close relationships to responsive caregivers, stable and supportive home environment), positive child attributes (i.e., effective emotional and behavioral regulation strategies, adaptable temperament), and positive community characteristics (i.e., high neighborhood quality, safe neighborhood, access to recreational centers) (Wright $\&$ Masten, 2006). Much research has already been done on many of these protective factors as it relates to adolescent substance use and overall health outcomes. However, involvement in physical activity as a potential positive activity and resource in the prevention of adolescent alcohol use has not been as well studied. The proposed study reflects this particular orientation by paying close attention to the potential influence of physical activity on depressive symptomatology and, in turn, adolescent alcohol use.

The concerns of the proposed study is that adolescence, in particular middle adolescence, is the period of life during which most youth initiate and escalate their alcohol use. In fact, teenage drinking is so commonplace that it may be considered a normative rather than an exceptional behavior. The study will examine the influence of potential protective factors (i.e. physical activity participation) on alcohol use during middle adolescence. While both alcohol and physical activity have been studied 
separately, minimal research has examined their relationship and mutual influence, either directly or indirectly (e.g., as mediated by depression). Moreover, there has been relatively little research exploring the role of developmental mediators (i.e. depression) that are sometimes present during this period of development. As a result, the study will assess how depressive symptomatology may influence the relationship between physical activity participation and alcohol use. The findings of this study will further understanding of what role protective factors (e.g., physical activity) can have on risk factors and behaviors during this time of the life course when individuals may be most amenable to public health intervention and thus aide in the prevention of further severe, negative and potentially lifelong consequences.

Risk and Resilience Perspective: Alcohol Use, Depression, and Physical Activity. In an attempt to understand the processes of risk and resilience, the constructs have been examined from two distinct levels: broad social contextual processes and individual psychological and biological processes (Compas, 2004). This study focused on the interaction between social processes offered through physical activity participation and individual psychological processes innate in depressive symptomatology.

Most research to date on adolescent alcohol use has focused on small subsets of risk factors. In fact, researchers have suggested that the most encouraging path to the development of effective strategies for the prevention of adolescent substance use problems is through a risk-focused approach (Hawkins, Catalano, and Miller, 1992). The knowledge resulting from research examining the relationship between risk and protective factors and substance use has contributed to significant advancements in understanding the etiology, assessment and prevention of substance abuse (Hawkins et 
al., 1992; Jessor, 1992). With these common factors identified what becomes the issue, particularly relevant to the proposed study, is how participation in physical activities can foster resilience among adolescents at risk for alcohol use.

In reference to this particular study, evidence supporting the mental-health benefits of physical activity continues to accumulate at an accelerated rate. There are now several hundred studies and over 30 narrative or meta-analytic reviews of research in this field. These studies have summarized the therapeutic potential physical activity involvement can have on clinical or subclinical depression or anxiety, and the use of physical activity as a means of acquiring attributes of resiliency through increased selfesteem, improved mood states, reduced state and trait anxiety, or resilience to stress (Fox, 1999). Early meta-analyses regarding depression and exercise in general populations (McDonald and Hodgson, 1991; North, McCullagh, and Tran, 1990) reported moderate to large effects for exercise on depression. However, these meta-analyses were limited to the inclusion of non-randomized clinical trials and studies where non-depressed populations were recruited.

A few years later, Craft and Landers (1998) attempted to provide more accurate answers regarding the relationship between depression and exercise. These studies only clinically depressed individuals. Thirty studies were included and analyses showed that exercise was associated with reduced depression (Effect size $=-0.72$ ). Additional analyses showed that the relationship between exercise and depression was not moderated by exercise program characteristics (e.g. duration, intensity, frequency and mode of exercise) or participants' characteristics (e.g. age, gender, and severity of 
depression). However, once again, the inclusion of observational studies and nonrandomized clinical trials limits the conclusions that can be drawn from this review.

Earlier research into the relationship between physical activity and mental health was based on cross-sectional and correlation analyses leaving open the possibility that the apparent health-promoting effects of exercise may simply indicate the effects of mood fluctuations on physical activity levels (Stathopoulou, Powers, Berry, Smits, \& Otto, 2006). More recent research using longitudinal and randomized clinical design have demonstrated that physical activity participation has consistent causal effects on promoting mental well-being in both clinical and non-clinical populations.

Particularly important to the proposed study is the limited amount of research on the potential efficacy of physical activity for the primary and secondary prevention of depression among adolescents. However, there is some research that has provided evidence that physical activity is beneficial to the development of adolescents (e.g. psychological, academic, behavioral functioning) (Barbara et al., 2001; Darling et al., 2005). Among the psychological benefits affected most by physical activity is mental health. Cross-sectional research suggests that participation in physical activity is inversely correlated with levels of depressive symptomatology (Goodwin, 2003; Motl et al., 2004). However, there is a lack of published studies on the effect of physical activity on depressive symptoms in non-clinically depressed samples of adolescents (Motl et al., 2004). Only two published studies on this association used non-clinically depressed samples and controlled for known confounders, including socioeconomic status (SES) and some measure of general health (Motl et al., 2004; Steptoe \& Butler, 1996). One study employed a longitudinal design and found that naturally occurring changes in 
physical activity were negatively related to changes in depression scores among adolescents over a 2-year period (Motl et al., 2004). Another study found a positive relation between an index of sport and vigorous activity and psychological well-being among adolescents in the United Kingdom (Steptoe \& Butler, 1996). The relations reported in these studies remained significant when the effect of confounding variables such as gender and socioeconomic status was controlled for.

Despite promising and consistent findings, research in this area has been plagued with methodological limitations, such as small sample sizes, and lack of control groups and random assignment. In addition, the studies mentioned consider physical activity participation at one point in time rather than across time. However, the associations suggest that regular exercise is related to low levels of indicators of mental illness, such as depression and depressed mood, among adolescents. Evidence suggests that reducing the prevalence of depression among adolescents can in fact protect against maladaptive behaviors such as substance abuse (Kirkcaldy et al., 2002; Nelson \& Gordon-Larsen, 2006).

While research exists on physical activity and mental health, the interactive effect of physical activity and alcohol use has not been studied at the same intensity. The relationship between risk (e.g. depressive symptomatology) and protective factors (e.g., physical activity) on adolescent alcohol use outcomes has not been thoroughly investigated (Arthur, Hawkins, Pollard, Catalano, Bagioni, 2002; Nelson \& GordonLarsen, 2006; Werch et al., 2003). Although research in this area is few and inconsistent, patterns among these variables suggest that physical activity may recruit processes, which confer enduring resilience to depression and ultimately to alcohol use. 


\section{Summary}

This study hopes to take individual puzzle pieces and demonstrate their correlations as well as link them together to provide a logical mechanism by which physical activity influences alcohol use outcomes. Processes of risk and resilience are at the center of our understanding of adaptive and maladaptive paths of development during adolescence. Understanding risk factors and processes of risk is central to the identification of those adolescents most in need of early intervention, whereas clarification of protective factors and processes of resilience can inform interventions to strengthen those at greatest risk.

If researchers are to capture the true essence of the relationship between physical activity participation and alcohol use, it is necessary to study the intervening mechanisms. One such mechanism may be found by turning to another prevalent health concern in adolescence, depression. This study assessed whether depressive symptomatology mediates the relationship between physical activity participation and adolescent alcohol use. This risk and resilience perspective provided the necessary structure for the complex interplay between physical activity participation and depressive symptomatology that potentially influences the development of alcohol use. In particular the study aims to test that earlier physical activity participation (Wave 1) is associated with one year later lower levels of depressed mood (Wave 2) and lower levels of alcohol use (Wave 2). This figure indicates two possible paths for an effect of physical activity on alcohol use (direct effect or indirect through the impact of depressive symptomatology).

Moreover, experts in the field of adolescent alcohol use disagree over the developmentally appropriate measurements of alcohol use outcomes, especially in the 
case of quantity-frequency indicators (Wagner 2008). The most commonly used measurement of alcohol use problems across adolescent alcohol treatment studies are quantity-frequency indicators. As an exploratory aim, this study attempts to assess the variability in the fit of the model according to three distinct definitions of alcohol use (quantity, frequency, and binge). Furthermore, some research suggests that the particular form of physical activity participation (e.g., organized sports participation, recreational sports involvement) makes a difference when attempting to understand the relationship between physical activity and adolescent alcohol use (Peretti-Watel \& Lorente, 2004; Moore \& Werch, 2005; Fredricks \& Eccles, 2006). Therefore, the study also examined the relationship between specific forms of physical activity participation and depressive symptomatology and alcohol use during middle adolescence.

This study recognized the potential for relationships among the variables in the model to be bi-directional. For example, high levels of depressed mood leading to low levels of physical activity and low levels of physical activity leading to high levels of depressed mood. However, natural changes in physical activity and depressed mood cannot be manipulated. Hence, the reason why this particular study relies on a longitudinal design, which can at least demonstrate and set the stage for which variable precedes the other in time.

Assessment of the variables included in this study was made utilizing the National Longitudinal Study of Adolescent Health, a longitudinal survey of a nationally representative sample of adolescents exploring health and health-risk behaviors. This dataset allowed for the temporal ordering necessary to establish mediation. In addition, the large, nationally representative sample of Add Health presented a unique opportunity 
to analyze the relationship between all three constructs as well as to analyze the relationships across time and produce results that have greater external validity than many previous studies. Below the specific research aims are delineated.

\section{Research Aims and Hypotheses}

Please see Figures 1 and 2 for a visual representation of the below mentioned hypothesized relationships between variables that were tested in this study. The below mentioned hypotheses are depicted in Figure 1 with frequency of alcohol use as the outcome variable. Figure 2 depicts the hypotheses with binge drinking as the outcome variable. Figures 1 and 2 exclude the covariates of sex, ethnicity, and parental income. This was done to avoid clutter but all covariates were included in all model tests. These models provide an initial conceptual framework for further research on the mechanism by which physical activity influences adolescent alcohol use.

Aim 1: To empirically test the influence of structured physical activity and unstructured physical activity on alcohol use (e.g., drinking frequency and frequency of binge drinking) among adolescents transitioning from middle school to high school, as a function of gender.

Hypothesis 1: Adolescents involved in more structured and unstructured physical activity will report less alcohol involvement (e.g., drinking frequency and frequency of binge drinking). The path coefficient linking structured and unstructured PA and alcohol involvement (drinking frequency and frequency of binge drinking) will be statistically significant and reflect an inverse relationship. 
Aim 2: To empirically test the influence of depressive symptomatology on alcohol use (e.g., drinking frequency and frequency of binge drinking) among adolescents transitioning from middle school to high school, as a function of gender.

Hypothesis 2: Adolescents with lower levels of depressive symptomatology will report less alcohol involvement (e.g., drinking frequency and frequency of binge drinking). The path coefficient linking depressive symptomatology and alcohol involvement (drinking frequency and frequency of binge dirnking) will be stastically significan and reflect a positive relationship.

Aim 3: To empirically test the mediating effects of depressive symptomatology on the relationship between structured and unstructured physical activity at Wave 1 and alcohol use (e.g., drinking frequency and frequency of binge drinking) at Wave 2, as a function of gender.

Hypothesis 3: Adolescents reporting more physical activity involvement will report less depression, which in turn will lead to less alcohol use (e.g., drinking frequency and frequency of binge drinking). The path coefficient between structured and unstructured physical activity and alcohol use (drinking frequency and frequency of binge drinking) from Wave 1 to Wave 2 will be significantly mediated by depressive symptomatology.

Aim 4: To empirically test the bi-directional effect between alcohol use (e.g., drinking frequency and frequency of binge drinking) and depressive symptomatology at Wave 2, by gender.

Hypothesis 4: Adolescents reporting less alcohol involvement (e.g., $\underline{\text { drinking }}$ frequency and frequency of binge drinking) would report less depressive 
symptomatology. The path coefficient linking alcohol involvement (e.g., drinking

frequency and frequency of binge drinking) and depressive symptomatology at Wave 2 will be statistically significant and reflect a positive relationship. 


\section{CHAPTER III. METHODOLOGY}

\section{Research Strategy}

This research used data from the National Longitudinal Study of Adolescent Health (Add Health), which is a large nationally representative sample of approximately 12,000 United States adolescents nested within 80 high schools. A prospective research design utilizing Waves 1 and 2 was used in order to examine the influence of physical activity participation on alcohol use and depressive symptomatology. The independent variables are assessed via the CES-D for depression and a standard physical activity behavior recall questionnaire assessing involvement in physical activity. The dependent variable was assessed via questions pertaining to alcohol involvement.

The following chapter provides a description of the research methods employed. The first section of the chapter provides an account of the sample design and data collection used to obtain the two waves of the National Longitudinal Study of Adolescent Health (Add Health) data. Next, the sample used to obtain the most complete and accurate information needed to achieve the specific aims of this study is delineated. Subsequently, detailed descriptions of the operationalization of all constructs used in the analysis, as well as, how the variables were measured are provided. The final sections of this chapter describe the statistical techniques used for the analysis, along with the specific analysis undertaken for each outcome examined in this study.

\section{The Add Health Data Set}

This study is based on a secondary analysis of existing data from Wave 1 and Wave 2 of the National Longitudinal Study of Adolescent Health (Add Health). Add Health was developed in response to a mandate from the U.S. Congress to fund a study 
focused on adolescents' health-related behaviors and forces that may influence $\begin{array}{lllll}\text { adolescents' } & \text { risk behaviors and health outcomes }\end{array}$ (http://www.cpc.unc.edu/projects/adhealth; Sieving et al., 2001). Add Health is considered the "largest, most comprehensive survey of adolescents ever taken" (National Longitudinal Study of Adolescent Health, 2003). The data set is longitudinal in nature in efforts to capture behavioral change over time. The data set is compromised of a nationally representative sample of adolescents in grades 7-12 in 1995 (Wave 1) who were followed up in a second interview one year later in 1996 (Wave 2).

Add Health utilized a school-based clustered sampling design, in which the clusters were sampled with unequal probability, in order to best screen for respondents of interest. The sampling frame was compromised of U.S. high schools and corresponding feeder schools, stratified by region, urbanicity, school type, ethnic composition, and enrollment size. The primary sampling unit of U.S. high schools was defined as including the $11^{\text {th }}$ grade and enrolling at least 30 students.

The Add Health study was designed to represent a nationally representative sample of U.S. high schools. From a sampling frame of 26,666 high schools, 80 high schools were selected from the Quality Education Data, Inc. database (QED). Of the 80 high schools selected, 52 included only grades nine through 12 and were supplemented by 52 feeder schools (i.e., schools that contained grade 7 and sent students to the selected high school) and 28 included grades seven through 12. High schools participating were asked to identify "feeder" schools. Subsequently, a single feeder school was randomly selected for each high school. Four schools had no eligible feeder school and 20 high schools were their own "feeder" schools. Thus, a total of 132 schools compromising 80 
school clusters or primary sampling units (PSU's) participated in the baseline survey. Seventy percent of the original schools sampled participated in the data collection; alternate schools within the same layer replaced those schools that refused to participate (Bearman, Jones, and Udry, 1997; Chantala and Tabor, 1999; Resnick et al., 1997). Figure 7 shows the overview of the Add Health longitudinal study design (Udry, Bearman, \& Harris, 2008).

\section{Add Health Data Participants and Collection Procedures}

The in school questionnaire was administered to all students in grades seven through 12 who were present at the time of data collection $(\mathrm{N}=90,118)$. The 45 -minute self-administered in-school questionnaire collected general descriptive information about the student's and parent's background, his or her peers, school life, school work and activities, and general health status. The in-school questionnaire did not ask about risk behavior. Subsequently, for more in-depth information home interviews were performed. A sample of adolescents were selected from all the students who completed an in-school questionnaire plus those who did not but who were listed on the school roster as eligible for selection. The in-home data questionnaire from Wave 1 and 2 were the ones utilized in this study.

The first wave of in-home data collection (Wave 1) occurred during the 19941995 school year and yielded an overall sample of 20,745 adolescents. The second wave of in-home data collection (Wave 2) occurred during 1996 and involved all adolescents interviewed at Wave 1, with the exception of those who were graduating seniors at Wave 1 and not part of the genetic sample, and the disabled subsample. Sixty-five siblings were also added to the genetic sample at Wave 2. The second wave of in-home data 
collection yielded an oversample of 14,736 cases. Active informed consent was obtained for these interviews.

The interviews were collected using computer-assisted personal interviews (CAPI) for purposes of confidentiality and accurate reporting of sensitive material. During the interview, questions were asked aloud by the computer via headphones and the adolescent responded by pressing an appropriate computer key on the laptop. Interviews were conducted in either English or Spanish. A parent or guardian, typically the mother, completed a parent questionnaire at Wave 1. The parent or guardian provided further information on family composition and adolescent's health history.

The in-home sample included a core probability sample $(n=12,105)$ of a nationally representative sample of teens in grades 7 through 12, and three supplemental over-samples: (1) ethnic minorities - African Americans with highly educated parents, Cubans, Puerto Ricans, and Chinese; (2) genetic relatedness to siblings; (3) adoption status; disability; and saturated schools (i.e. $100 \%$ of the student body was sampled). The response rate for participation in the Add Health surveys was $78.9 \%$ at Wave 1 and $88 \%$ at Wave 2. In efforts to accommodate bias due to oversampling, non-response, and poststratification grand sample weights were derived to adjust for the sample design and response rates are applied in all analysis.

\section{Study Sample}

This analysis is based on data collected from the first and second Wave of Add Health data collection. Eighth graders during Wave 1 were followed through the transitional period between middle and high school to ninth grade in Wave 2. This is an underexplored and unique period in adolescent development. Additionally, in order to 
ascertain socio-demographic characteristics, such as parents' income and other indicators of socioeconomic status, some data provided by the parent interview was used. This section reports unweighted descriptive statistics. However, Table 1 and 2 report both weighted and unweighted values.

The sample for the proposed research consisted of 2,054 participants from Waves 1 and 2 of the original parent study. The sample included males and females in the $8^{\text {th }}$ grade with a mean age of $14.02(S D=.68)$. Table 1 provides all key sociodemographic information for participants in the current study. In addition, Table 2 reports the weighted and un-weighted means and standard deviations of all measures used in this analysis for the total study population, as well as, by sex. The mean age of the participants in this current study incorporates the transition from middle to high school. As shown in Table 1, of the 2,054 participants $51.1 \%$ were female. The racial ethnic backgrounds of the adolescents assessed were 58.2\% White, 24\% African American, 11.7\% Hispanic, and $6.1 \%$ other. The parents of study participants reported average annual income of $34,450.00(S D=23,350.00)$. In addition, the majority of the study participants' parents had obtained a high school degree.

Regarding human subjects protection, the data used were archival with identifying information removed. The data was acquired through the Data Sharing for Demographic Research, a project of the Inter-University Consortium for Political and Social Research (ICPSR). Strict data security measures were implemented and approved by the ICPSR before data was obtained. All research methods for this study were approved by the Institutional Review Board (IRB) at Florida International University. 


\section{Measures}

Adolescent Physical Activity. The Add Health in-home survey assessed physical activity across Waves 1 and 2. The questionnaire included a standard physical activity behavior recall that is similar to other self-reported questionnaires that have been used and validated in other large-scale published epidemiological studies (Sallis et al., 1993; Andersen et al., 1998; Knutson, 2005; Nelson, Gordon-Larsen, Adair, \& Popkin, 2005).

Physical activity in this study was measured by three items using a 7-day recall (times/week); whereby participants reported the frequency in which they engaged in three different types of activities: (1) times roller-blading/cycling in past week, (2) active sports such as soccer, swimming etc., and (3) exercise such as jogging or dancing. The questions were worded as such, "During the past week, how many times did you," followed by a list of activities, such as walking, basketball, softball, and so forth. Possible responses included 0 (none), 1 ( 1 to 2 times per week), 2 ( 3 to 4 times per week), and 3 (5 or more times per week) (See Appendix A). In addition, for purposes of this study, secondary analyses will be run on the type of physical activity (fitness activities equivalent to roller-blading, skate-boarding, etc., and organized sport equivalent to participation in team sports such as football, basketball, soccer, etc.). In order to explore potential variation based on specific type of physical activity (e.g., organized sport versus recreational hobbies), two separate variables were created: Physical Activity Structured (PA_S)and Physical Activity Unstructured (PA_US). The PA_US variable was created by averaging the responses to the two questions assessing recreational hobbies; "During the past week, how many times did you go rollerblading, roller-skating, skate-boarding, 
or bicycling?" and "During the past week, how many times did you do exercise, such as jogging, walking, karate, jumping rope, gymnastics or dancing?" respectively.

Adolescent Depressive Symptomatology. Depressive symptomatology was assessed by the Add Health in-home survey at Waves 1 and 2 using 19-items of the Center for Epidemiological Studies Depression Scale - CES-D; Radloff, 1977). The CES-D is a well validated and widely used measure of symptoms of depression within community samples.

The CES-D taps into adolescents' feelings of distress. In the Add Health data set, respondents reported the frequency of experiencing each depressive symptom within the previous week, with responses ranging from (0) "never or rarely" to (3) "most of the time;" positive items such as "you enjoyed life" will be reversed scored. From the CESD survey, symptoms included- 'You were bothered by things that don't usually bother you," "You felt that you could not shake off the blues even with the help of family and friends," and "You felt lonely." The original CES-D scale can be found in Appendix B Depressive symptomatology scores were obtained by summing the item scores to yield a total score. Scores for items expressing positive affect were reverse-coded to make them consistent with the scores for items assessing depressive symptoms. Total scores could range from 0 to 57, with the higher scores indicating the presence of more symptomatology.

The adult version of the CES-D have been demonstrated to be reliable in both population-based and clinical samples of adults $(\alpha=.85 \& \alpha=.90$, respectively) (Radloff, 1977). In support of the proposed research, the appropriateness of the CES-D scale to gauge levels of emotional distress in community surveys of adolescents, as well 
as the comparability of adolescent and adult CES-D scores, has also been established ( Roberts 1995b; Wight, Sepulveda, and Aneshensel, 2004). Good reliability with a Cronbach's alpha ranging from .85 to .91 has been demonstrated in the adolescent literature (Aseltine, Gore, \& Colten, 1994; Schoenbach, Kaplan, Wagner, Grimson, \& Miller, 1983; Windle, 1992). In the present sample, the Cronbach's alpha for the CES-D in Wave 1 was .86 and .87 for Wave 2.

Adolescent Alcohol Use. The Add Health alcohol use measure was a result of direct collaboration with the National Institute on Alcohol Abuse and Alcoholism (NIAAA). Investigators from Add Health asked for the most state of the art indicators of alcohol use behaviors among adolescents. As a result, the most valid and reliable questions used in other larger epidemiological studies were utilized to assess adolescent alcohol use. The questions have been found to have acceptable levels of reliability appropriate for use with future research studies using the Add Health public use data set (Sieving et al., 2001). Alcohol use was a categorical measure constructed from a series of questions related to current (past year) alcohol use. Respondents completed a detailed section of the in-home questionnaire at Waves 1 and 2 related to overall alcohol use that included 18 items assessing general patterns and frequency of alcohol use, as well as problems related to alcohol consumption over the past year.

Two items asked of the adolescents at Wave 1 and 2 that focused on alcohol use were used to construct both the frequency and binge drinking variables in this study. All participants during Wave 1 and 2 were asked if they ever drank an alcoholic beverage: “...beer, wine or liquor - not just a sip or taste of someone else's drink" ...2-3 times in our life..." If the response to this item was affirmative, adolescents were then asked to 
report the frequency of current use, defined as in the past 12 months. Frequency of drinking was assessed with the question, “...on how many days during the past year did you drink alcohol?" Subsequently, if the individual responded positively to drinking in the past year, participants were then asked questions pertaining to binge drinking. Binge was assessed by asking the individual, “...on how many days during the past year did you drink five or more drinks in a row?".. Response categories ranged from 1 to $7 ; 1=$ "almost every day," $2=$ "3-5 days a week," $3=$ " $1-2$ days a week," $4=$ " $2-3$ days a month," $5=$ "once a month or less," $6=$ " 1 or 2 days in past 12 months," and $7=$ "never."

The question related to drinking five or more drinks on occasion is commonly used in community surveys of adolescent alcohol use to assess binge drinking (SAMHSA, 1999). The current study used the following alcohol use indicators; number of days of use, and number of days of consuming five or more drinks in a single occasion to construct an assessment of alcohol use among the Add Health participants at each time point. A list of the alcohol-related items from the Add Health in-home survey (Wave 1 and 2) is given in Appendix $\mathrm{C}$ with an asterisk representing the questions that were used in the current study.

\section{Summary of Analysis Plan}

Several analytic approaches were incorporated into the proposed research study to investigate the associations between physical activity, depressive symptomatology and frequency and binge alcohol use across time. First descriptive statistics and correlations among the study variables were run to depict the occurrence of both depressive symptomatology, frequency and binge alcohol use, and the extent to which physical activity participation influences the pathway. Secondly, the influence of physical activity 
on adolescent depressive symptomatology and alcohol use (frequency and binge) were examined within a structural equation modeling framework.

As described earlier, two conceptual structural equation models were created to study the relationships between the constructs (see Figure 1 and 2). The first model examined the associations between physical activity and depressive symptomatology and frequency of alcohol use. The second model examined the associations between physical activity and depressive symptomatology and binge alcohol use. Multiple regression techniques and structural equation modeling (SEM) with robust methods of maximum likelihood analysis was employed, through the use of SPSS and M Plus (version 6.0) software respectively, to help gain additional insight into the "fit" potential of the proposed causal models and the strength of variable relationships (e.g., structural parameter estimates). In order to maintain the accuracy of data entered, data was crosschecked for errors such as out of range values and missing data as well as outliers. Transformation and computation of variables were conducted when necessary.

Missing Data. Missing data was expected to be minimal, infrequent and not problematic for most variables. The Add Health Study's use of weighted data helps to compensate for those who were excluded because of missing values (Raghunathan, 2004). Weighted data is effective in eliminating non-response bias. Sampling weights were incorporated in the Add Health data set to allow for the following: the sample totals to serve as estimates of population totals, compensate for differences in selection probabilities across different members of a sample, compensate for differences in response rates across different groups, and to adjust for possible fluctuations of the composition of the sample from the composition of the sample as a whole (Tourangeau \& 
Shin, 1999). For further information about the Add Health weighting procedures, see Tourangeau and Shin (1999). Given any missing data, parameter estimates and model tests will be pursued in the context of Full Information Maximum Likelihood (FIML) methods as implemented in M Plus. Given any missing data, parameter estimates and model tests will be pursued in the context of Full Information Maximum Likelihood (FIML) methods as implemented in M Plus.

Outliers. Analysis of outliers were undertaken prior to all formal analyses. Standard methods for outlier detection (e.g., analysis of leverage statistics and dfBetas) and use of graphical approaches were applied. The analyses were both non-model based and model based. For the former, multivariate outliers were identified by examining leverage indices for each individual and defining an outlier as a leverage score four times greater than the mean leverage. An additional set of outlier analyses will be pursued using model-based outlier analysis. Model based outliers will be examined using limited information regression analyses for each of the linear equations dictated by the various path models tested (Bollen, 1996). The dfbeta values for each individual relative to each path coefficient will be examined to isolate unusually influential individuals in parameter estimation. An outlier was defined as anyone with dfbetas three times larger than the standard error of a coefficient.

Non-normality. Multivariate normality is typically not an issue in complex data sets such as Add Health. If non-normality appears to be problematic, then a robust estimation method based on the Huber-White estimator will be used as implemented in M Plus. In addition, univariate indices of skewness and kurtosis will be examined to determine if the absolute value of any of these indices is greater than 2.0. 
Bidirectionality. In order to accommodate the bidirectionality included in the analysis between depression and frequency of alcohol use and binge, number of friends who drink was included in model as an instrumental variable with a direct path to frequency of alcohol use and binge in models 1 and 2 respectively (see Figures 1 and 2).

Testing for mediation. According to Hair et al., (2006) mediation is defined as when "a third variable/construct intervenes between two other related constructs" ( $p$. 866). They state that "theoretically, a mediating construct facilitates the relationship between the two other constructs involved" (p. 867). In regard to mediational analyses, the logic of the joint significance test was used as a basis for stating mediation (MacKinnon, Lockwood, Hoffman, West \& Sheets, 2002). This is a superior test of mediation relative to the traditional approach of Baron and Kenny (1986). The logic is that if all path coefficients in a mediated chain are statistically significant, then mediation is declared.

Testing for moderation. Moderation can be defined as the "effect of a third variable or construct changing the relationship between two related variables/constructs. That is, a moderator means the relationship between variables changes based on the amount of another variable added to the model" (Hair et al., 2006). In order to test for moderation, product terms were created. Formal interaction analyses were pursued to examine any significant differences in the model path coefficients as a function of gender. Product terms were introduced into the model for all relevant paths as noted in Figures 1 and 2. Single degree of freedom interaction contrasts were performed comparing the effects of each of the exogenous, as well as the mediator variables on each of the two alcohol outcome variables (frequency and binge) as a function of gender. 
Indices of Fit. Following the recommendations of Bollen and Long (1993), a variety of global fit indices were used, including indices of absolute fit, indices of relative fit and indices of fit with a penalty function for lack of parsimony. These include the traditional overall chi square test of model fit (which should be statistically nonsignificant), the Root Mean Square Error of Approximation (RMSEA; which should be less than 0.08 to declare satisfactory fit), the p value for the test of close fit (which should be statistically non-significant), the Comparative Fit Index (CFI; which should be greater than 0.95); and the standardized root mean square residual (which should be less than $0.05)$.

In addition to the global fit indices, more focused tests of fit were pursued. These included examination of the standardized residual covariances (which should be between -2.00 and 2.00) and modification indices (which should be less than 4.00). Care was taken to ensure there was no specification error. 


\section{CHAPTER IV. RESULTS}

This study, utilizing structural equation modeling (SEM), aimed to examine, (1) the influence of independent variables structured and unstructured physical activity, measured at Wave 1, and Depressive Symptomatology measured at Wave 2 on Wave 2 outcome variables, frequency of alcohol use and frequency of binge drinking; (2) whether depressive symptomatology mediates the relationship between physical activity (structured and unstructured) and frequency of alcohol use and binge drinking, respectively, (3) the bidirectional relationship between frequency of alcohol use and frequency of binge drinking and depressive symptomatology at Wave 2. The conceptual models examining these relationships are depicted by Figures 1 and 2 . As a final Research Aim, both conceptual models were examined using gender as a moderator. Moderation analyses were conducted using a product term, which is reviewed in further detail in the primary analysis section of the results.

This chapter includes the results for the current study. The data analytic plan for the present study consisted of preliminary analyses that included: 1) obtaining descriptive statistics of the sample, 2) outlier analyses, 3) missing data analyses, and 4) checks for non-normality. A review of the results from these preliminary analyses is outlined below. The study models were just identified, therefore, the primary analyses consisted of examining the models in terms of their corresponding path coefficients and standard errors. The results of the hypotheses relative to each of the study's aims are presented below. 


\section{Preliminary Analyses}

Descriptive Statistics. Basic descriptive statistics (both weighted and unweighted) were calculated for all of the variables in the models (including covariates and the instrumental variable) (see Tables 1 and 2). Table 2 is stratified by gender because of the study's emphasis on the interaction between gender and study variables. The descriptive statistics suggest that males engaged in more structured and unstructured physical activities, frequency of alcohol use, and binge drinking than females, and females exhibited more depressive symptomatology than males. The descriptive statistics support the need to analyze the interaction between these variables and gender on drinking outcomes (frequency of alcohol use and frequency of binge drinking). This analysis is discussed in further detail below.

Missing data. Missing data for each of the study variables ranged from $.05 \%$ to 66.89\%. The Add Health Study's use of weighted data helps to compensate for those who were excluded because of missing values (Raghunathan, 2004). Weighted data is effective in eliminating non-response bias. Missing data bias was assessed by computing a dummy variable reflecting the presence or absence of missing data for each variable, which was correlated with all other variables in the model as well as demographic variables. Bivariate correlations using Pearson's $r$ were calculated among the study variables. There were no systematic patterns observed in the missing data. Full information maximum likelihood (FIML) was employed in SEM to accommodate the missing data. It is important to note that FIML has been shown to be quite robust to violations of normality (Enders, 2010). 
Outliers. Analyses of both non-model based and model based outliers were pursued prior to conducting the primary analyses. Standard methods for outlier detection (e.g., analysis of leverage statistics and df betas) were applied. The leverage score was calculated for each individual, with an outlier defined as a leverage score three times greater than the mean (Jaccard \& Wan, 2003). The mean leverage score across respondents was .012 . There were no outliers evident using this criterion. Model based outliers were examined using limited information regression analyses for each of the linear equations dictated by the various path models tested (Bollen, 1996). This involved regressing each endogenous variable onto the variables of which the endogenous variables were assumed to be a linear function. Standardized dfbetas for each individual relative to each path coefficient were also examined to isolate especially influential individuals in parameter estimation. An outlier was defined as anyone with an absolute standardized dfbeta larger than 1.0. No model based outliers were present in these analyses using this approach.

Non-normality. To account for any possible non-normality present in the data, SEM analyses were pursued in MPlus by using an estimator (MLR) robust to violations of normality based on the Huber-White algorithm. Data were screened for any nonnormality (e.g., skewness and kurtosis). Examination of univariate indices of skewness and kurtosis were conducted to determine if any of the indices exceeded an absolute value of 2.0. There was no significant skewness or kurtosis evident based on this criterion (see Table 3). It is important to note that through the use of the robust estimation method of Hubert-White, non-normality is not a significant issue within a complex data set such as Add Health. 


\section{Primary Analyses}

Primary analyses were conducted after determining that there were no outliers and that the issues of missing data and non-normality could be adequately accommodated for through the use of FIML and MLR, respectively. The primary analytic approach involved the use of SEM using the Mplus software (version 6.0). The proposed models seen in Figures 1 and 2 tested a series of research aims regarding the influence of physical activity, both structured and unstructured, on frequency of alcohol use and binge drinking, respectively. All paths in the model were evaluated using gender as a moderator. Consistent with findings in the literature and from the Add Health data set from which the study sample was derived, it was imperative to look at study aims as a function of gender.

By testing for interaction effects, it can be determined if the strength of the relationships between the study variables vary as a function of gender. Interaction effects in the SEM analyses were modeled using product terms, as discussed in Jaccard, Turrisi, and Wan (1990) and Jaccard and Wan (1996). Product terms were introduced into the model for all relevant paths. Single degree of freedom interaction contrasts were performed comparing the effects of each of the exogenous variables, as well as the mediator variable on each of the two alcohol outcome variables (frequency and binge) as a function of gender. These product terms reflect the differences in slopes between males and females among the hypothesized relationships. Given the addition of these product terms in the analyses, all continuous variables were mean centered for ease of interpretation of path coefficients (see Jaccard and Turrisi, 2003). 
Model fit. Two separate models were created for methodological reasons (see Figures 1 and 2). It was not possible to examine the bidirectional relationship between depressive symptomatology at Wave 2 and both frequency of alcohol use and binge drinking within the same model without the need for two instrumental variables. Given that only one instrumental variable was identified from the data set (number of friends who drink at Wave 2), it was imperative to create two separate conceptual models (see Figures 1 and 2). As described earlier, all paths in the models were analyzed as a function of gender. Only the interaction between gender and depressive symptomatology at Wave 2 was significant (see Tables 5, and 6 and Figures 5 and 6). As such, the models were then trimmed of the other interaction contrasts. Given the significant interaction with gender and depressive symptomatology on alcohol use (frequency and binge) the results are presented in four separate models (see Figures 3, 4, 5, and 6). The models were just identified; $\chi^{2}=0 ; \mathrm{CFI}=1.00 ; \mathrm{RMSEA}=.00$. This type of fit occurs when the number of free parameters exactly equals the number of known values; therefore it is a model with zero degrees of freedom. Although not illustrated in all four models, covariates were included for all endogenous variables, including age, race/ethnicity, parental income, and parental level of education, and all Wave 1 variables (Wave 1 depressive symptomatology, Wave 1 frequency of alcohol use, and Wave 1 frequency of binge drinking).

Results for the final models are presented in Figures 3, 4, 5, and 6. All exogenous variables are assumed to be correlated in all models. The values included on the paths are unstandardized path coefficients. Only significant path coefficients are reported in the figures. All unstandardized path coefficients, $p$ values, and $95 \%$ confidence interval 
values are listed in Tables 4, 5, 6, and 7. Reviewed below are the study aims, hypotheses, and results incorporating gender for both the frequency and binge drinking models. Results for the models using frequency of alcohol use as an outcome variable are presented first, followed by the results for the models using binge drinking as an outcome variable.

\section{Research Aims and Hypotheses}

Research Aim 1 was to empirically test the influence of structured physical activity and unstructured physical activity on alcohol use (e.g., $\underline{\text { drinking frequency }}$ and frequency of binge drinking) among adolescents transitioning from middle school to high school, as a function of gender.

Hypothesis 1: Adolescents involved in more structured and unstructured physical activity will report less alcohol involvement (e.g., drinking frequency and

frequency of binge drinking). Paths $a$ and $b$ in the structural models presented in Figures 3, 4, 5, and 6 were used to test this hypothesis.

\section{Results for Frequency of Alcohol Use Models}

Utilizing SEM for this analysis, all unstandardized path coefficients for both males and females are reported in Tables 4 and 5 for Figures 3 and 4 . Paths of interest in Figures 3 and 4 are $a$ and $b$. As shown in Figures and 3 and 4 and Tables 4 and 5 among males and female adolescents transitioning from middle school to high school, no statistically significant relationships existed between structured or unstructured physical activity and frequency of alcohol use. The model is not consistent with the hypothesis that adolescents with less participation in physical activities, both structured and unstructured, will report a higher frequency of alcohol use. 


\section{Results for Binge Models}

Utilizing SEM for this analysis, all significant unstandardized path coefficients for the male and female models are reported in Tables 6 and 7 for Figures 5 and 6. Paths of interest in Figure 5 and 6 are $a$ and $b$. As shown in Figures 5 and 6 and Tables 6 and 7, statistically significant relationships existed between structured physical activities and binge drinking. However, results were not consistent with the hypothesis that adolescents participating in less physical activities would report more binge drinking. Both males and female adolescents who reported engaging in structured physical activities were more likely to binge drink (males path $a=.11, p<.05,95 \% \mathrm{CI}=.00$ to .21 ; females path $a=$ $.13, p<.01,95 \% \mathrm{CI}=.04$ to .23$)$. The same was not found to be the case with unstructured physical activities, where no significant relationship was found between unstructured physical activity participation and binge drinking for either males or females. Thus, there was no support found for the hypothesis that greater physical activity participation, either structured and/or unstructured, results in less alcohol involvement (e.g., binge drinking).

Research Aim 2 was to empirically test the influence of depressive symptomatology on alcohol use (e.g., drinking frequency and frequency of binge drinking) among adolescents transitioning from middle school to high school, as a function of gender.

Hypothesis 2: Adolescents with lower levels of depressive symptomatology will report less alcohol involvement (e.g., drinking frequency and frequency of binge drinking). Path $e$ in the structural models presented in Figures 3, 4, 5, and 6 were used to test this hypothesis. 


\section{Results for Frequency of Alcohol Use Models}

Utilizing SEM for this analysis, all significant unstandardized path coefficients for the male and female models are reported in Tables 4 and 5 for Figures 3 and 4. Path $e$ is of interest in Figures 3 and 4. A significant interaction effect by gender was found for the path from depressive symptomatology to frequency of alcohol use at Wave 2 (path $e$ in the model), suggesting that the path coefficient of frequency of alcohol use at Wave 2 on depressive symptomatology at Wave 2 differed for males and females. This was the only significant interaction found in the model when using gender as a moderator. Specifically, the figures show that for both males and females, as depressive symptomatology increased, the frequency of drinking decreased (males path $e=-.10, p$ $<.05,95 \% \mathrm{CI}=-.19$ to -.01 ; females path $e=-.06, p<.05,95 \% \mathrm{CI}=-.12$ to -.00$)$. The model is not consistent with the hypothesis that adolescents with higher levels of depressive symptomatology will report a higher frequency of alcohol use.

\section{Results for Binge Models}

Utilizing SEM for this analysis, all significant unstandardized path coefficients for the male and female models are reported in Tables 6 and 7 for Figures 5 and 6 . Path $e$ is of interest in Figures 5 and 6. A significant interaction effect by gender was found for the path from depressive symptomatology to binge drinking Wave 2 (path $e$ in the model), suggesting that the path coefficient of frequency of binge drinking at Wave 2 on depressive symptomatology at Wave 2 differed for males and females. This was the only significant interaction found in the model when using gender as a moderator. Specifically, the figures show that for males, the path coefficient (path $e$ ) was -.10 ( $p<$ $.05,95 \% \mathrm{CI}=-.19$ to -.01$)$ whereas for females it was $-.06(p<.05,95 \% \mathrm{CI}=-.12$ to - 
$.00)$. Thus for males, as depressive symptomatology increased, the frequency of binge drinking decreased. In contrast, there was no significant relationship between depressive symptomatology and frequency of binge drinking for females. The model is not consistent with the hypothesis that adolescents with higher levels of depressive symptomatology will report a higher frequency of binge drinking.

Research Aim 3 was to empirically test the mediating effects of depressive symptomatology on the relationship between structured physical activity and unstructured physical activity at Wave 1 and alcohol use (e.g., frequency and frequency of binge drinking) at Wave 2, as a function of gender.

Hypothesis 3: Adolescents reporting more physical activity involvement will report less depressive symptomatology, which in turn will lead to less alcohol use (e.g., drinking frequency and frequency of binge drinking).

With respect to mediation, the joint significance test was used as recommended by MacKinnon et al. (2002) and as such, the paths of interest in Figure 3, 4, 5, and 6 are $c$ and $e$, and $d$ and $e$. Path $c$ represents the influence of structured physical activity on depressive symptomatology; path $e$ represents the influence of depressive symptomatology on alcohol use (frequency and binge); path $d$ represents the influence of unstructured physical activity on depressive symptomatology. In accordance with the recommendations of MacKinnon et al., paths $c$ and $e$ need to be statistically significant to conclude that depressive symptomatology mediates, to some extent, the relationship between structured physical activity and alcohol use outcomes (frequency and binge). Paths $d$ and $e$ need to be statistically significant to conclude that depressive 
symptomatology mediates, to some extent, the relationship between unstructured physical activity and alcohol use outcomes (frequency and binge).

\section{Results for Frequency Models}

SEM was utilized to examine the extent to which the relationship between participation in physical activity (structured and unstructured) and frequency of alcohol use is mediated by depressive symptomatology. All significant unstandardized path coefficients for the male and female models are reported in Tables 4 and 5 for Figures 3 and 4. Paths $c$ and $e$ and $d$ and $e$ are of interest in Figures 3 and 4. The path from structured physical activity to depressive symptomatology was not significant (males path $c=-0.23, p=.17,95 \% \mathrm{CI}=-0.08$ to 0.11 ; females path $c=-0.23, p=.17,95 \% \mathrm{CI}=.56$ to .10). In addition, the path from unstructured physical activity to depressive symptomatology was not significant (males path $d=-.03, p=.91,95 \% \mathrm{CI}=-.54$ to .48 ; females path $d=-.03, p=.91,95 \% \mathrm{CI}=-.07$ to .31 ). As noted in the above analyses the path from depressive symptomatology to frequency of alcohol use was significant for both males and females (males path $e=-.10, p<.05,95 \% \mathrm{CI}=-.19$ to -.01 ; females path $e=-.06, p<.05,95 \% \mathrm{CI}=-.12$ to -.00 ). However, because the paths from physical activity (structured and unstructured) to depressive symptomatology were not significant, there is no evidence for mediation. Thus there is no support for the hypothesis that depressive symptomatology is a mediator of the relationship between physical activity (structured and unstructured) and frequency of alcohol use.

\section{Results for Binge Models}

SEM was utilized to examine the extent to which the relationship between participation in physical activity (structured and unstructured) and frequency of binge 
drinking is mediated by depressive symptomatology. All significant unstandardized path coefficients for the male and female models are reported in Tables 6 and 7 for Figures 5 and 6. Paths $c$ and $e$ and $d$ and $e$ are of interest in Figures 5 and 6. The path from structured physical activity to depressive symptomatology was not significant (males path $c=-.35, p=.10,95 \% \mathrm{CI}=-0.76$ to 0.07 ; females path $c=-0.35, p=.10,95 \% \mathrm{CI}=-.76$ to .07). In addition, the path from unstructured physical activity to depressive symptomatology was not significant (males path $d=-.04, p=.87,95 \% \mathrm{CI}=-.76$ to .07 ; females path $d=-.04, p=.87,95 \% \mathrm{CI}=-.54$ to .45 ). As noted in the above analyses the path from depressive symptomatology to frequency of binge drinking was significant for males but not for females (males path $e=-.12, p<.05,95 \% \mathrm{CI}=-.22$ to -.01 ; females path $e=-.07, p=.053,95 \% \mathrm{CI}=-.14$ to .00$)$. However, because the paths from physical activity (structured and unstructured) to depressive symptomatology were not significant, there is no evidence for mediation. Thus there is no support for the hypothesis that depressive symptomatology is a mediator of the relationship between physical activity (structured and unstructured) and frequency of binge drinking.

Research Aim 4 was to empirically test the bidirectional relationship between alcohol use (e.g., drinking frequency and frequency of binge drinking) and depressive symptomatology at Wave 2, by gender.

Hypothesis 4: Adolescents reporting less alcohol involvement (e.g., drinking frequency and frequency of binge drinking) would report less depressive symptomatology. In order to accommodate the bidirectionality included in analyses between depressive symptomatology and adolescent alcohol use (frequency and binge), number of friends who drink at Wave 2 was included in 
the models as instrumental variable with a direct path to frequency of alcohol use and a direct path to frequency of binge drinking, respectively.

\section{Results for Frequency Models}

SEM was used to assess whether adolescents reporting higher frequency of alcohol use would report more depressive symptomatology and whether adolescents reporting more depressive symptomatology would report higher frequency of alcohol use. The significant unstandardized path coefficients are reported in Figures 3 and 4 and all unstandardized path coefficients are reported in Tables 4 and 5. Paths $e$ and $f$ of Figures 3 and 4 are of interest in addressing Aim 4. Path $e$ represents the relationship between depressive symptomatology and frequency of alcohol use. Path $f$ represents the relationship between frequency of alcohol use and depressive symptomatology. As noted in Aim 2, the path from depressive symptomatology to frequency of alcohol use (path $e$ ) was moderated by gender and a significant relationship was found between depressive symptomatology and frequency of alcohol use for both males and females. However, both males and females who reported more depressive symptomatology reported less frequency of alcohol use, which was not consistent with Hypothesis 2. Path $f$ indicates a significant and positive relationship between frequency of alcohol use and depressive symptomatology. For both males and females, as frequency of drinking increased, depressive symptomatology increased (males path $f=1.36, p<.01,95 \% \mathrm{CI}=.46$ to 2.26 ; females path $f=1.36, p<.01,95 \% \mathrm{CI}=.46$ to 2.26 ). This finding is consistent with the hypothesis that adolescents presenting higher frequency of alcohol use would report more depressive symptomatology. 


\section{Results for Binge Models}

SEM was used to assess whether adolescents reporting higher frequency of binge drinking would report more depressive symptomatology and whether adolescents reporting more depressive symptomatology would report higher frequency of binge drinking. The significant unstandardized path coefficients are reported in Figures 4 and 6 and all unstandardized path coefficients are reported in Tables 6 and 7. Paths $e$ and $f$ in Figures 5 and 6 are of interest in addressing Aim 4. Path $e$ represents the relationship between depressive symptomatology and frequency of binge drinking. Path $f$ represents the relationship between frequency of binge drinking and depressive symptomatology. As noted in Aim 2, the path from depressive symptomatology to frequency of binge drinking (path $e$ ) was moderated by gender and a significant relationship was found between depressive symptomatology and frequency of binge drinking for males but not for females. However, males who reported more depressive symptomatology had lower frequencies of binge drinking, which was not consistent with Hypothesis 2. Path $f$ indicates a significant and positive relationship between frequency of binge drinking and depressive symptomatology. For both males and females, as frequency of binge drinking increased, depressive symptomatology increased (males path $f=1.33, p<.01,95 \% \mathrm{CI}=$ .52 to 2.13 ; females path $d=1.328, p<.01,95 \% \mathrm{CI}=.52$ to 2.13 ). This finding is consistent with the hypothesis that adolescents presenting a higher frequency of binge drinking would report more depressive symptomatology.

\section{Post Hoc Analyses}

Given that a significant relationship between frequency of alcohol use and binge drinking (respectively) and depressive symptomatology was evident, post hoc analyses 
were conducted. Analyses were conducted to examine whether frequency of alcohol use and/or frequency of binge drinking mediated, to some extent, the relationship between physical activity (structured and unstructured) and depressive symptomatology. Given that physical activity (structured and unstructured) was not found to be significantly associated with frequency of alcohol use (see Figures 3 and 4 and Tables 4 and 5), there was no evidence of mediation according to the criteria for the joint significance tests.

However, this was not found to be the case with the models for frequency of binge drinking for males and females. In contrast to the hypothesized meditation of depressive symptomatology, binge drinking was found to be a mediator between structured physical activity participation and depressive symptomatology. This is shown in paths $a$ and $f$ in Figures 5 and 6. Path $a$ represents the influence of structured physical activity on binge drinking, and path $f$ represents the influence of binge drinking on depressive symptomatology. In accordance with MacKinnon et al., paths $a$ and $f$ need to be statistically significant to conclude mediation. Paths $a$ and $f$ were found to be statistically significant and positive. For both males and females, adolescents reporting more participation in structured physical activity also reported more binge drinking (Males path $a=.11, p<.05,95 \% \mathrm{CI}=.00$ to .21 , Females path $a=.13, p<.01, \mathrm{CI}=.04$ to .23). For both males and females, adolescents reporting more binge drinking also reported more depressive symptomatology (Males path $f=1.33, p<.01,95 \% \mathrm{CI}=.52$ to 2.13; Females path $f=1.33, p<.01,95 \% \mathrm{CI}=.52$ to 2.13 ). Binge drinking was not found to be a mediator for unstructured physical activity as indicated by the nonsignificant path $b$. 


\section{Supplemental Analyses}

In addition to the above model analyses, supplementary analyses were conducted to explore potential problems of model misspecification. As per Jaccard and Wan (2003) traditional regression methods were used in conjunction with product terms to test for possible interaction effects between predictors of each endogenous variable. No significant interactions were found. This meant that no misspecifications of the main explanatory variables (physical activity structured and unstructured) and other covariates were found that could have affected tests of the associations between the explanatory variables and the response variable (frequency of alcohol use and binge drinking).

The standardized residuals indicate the proportion of unexplained variance in the endogenous variables. For males, the structured and unstructured physical activity variables were able to account for $34 \%$ of the variance in depressive symptomatology and $14 \%$ of the variance in frequency of alcohol use. For females, the variables were able to account for $34 \%$ of the variance in depressive symptomatology and $18 \%$ of the variance in frequency of alcohol use. Thus, for both males and females, a moderate and roughly equal proportion of the variance in the outcomes was accounted for.

The standardized residuals indicate the proportion of unexplained variance in the endogenous variables. For males, the variables were able to account for $34 \%$ of the variance in depressive symptomatology and $22 \%$ of the variance in binge drinking. For females, the variables were able to account for $34 \%$ of the variance in depressive symptomatology and $26 \%$ of the variance in binge drinking. Thus, for both genders, a moderate and roughly equal proportion of the variance in the outcomes was accounted for. 


\section{Summary of Results}

The hypotheses regarding the influence of physical activity (structured and unstructured) on adolescent alcohol use (frequency of alcohol use and binge drinking) were not supported. Engaging in structured physical activity appeared to contribute to greater binge drinking among adolescents. Thus, rather than showing that physical activity is a protective factor, the findings suggest that engaging in structured physical activity places adolescents at risk for binge drinking. Furthermore, no significant relationships, positive or negative, were found for the influence of physical activity (structured and unstructured) on frequency of alcohol use. For the hypothesis regarding depressive symptomatology as a mediator of the relationship between physical activity (structured and unstructured) and alcohol use (frequency and binge) was not supported. The findings actually revealed binge drinking as a mediator of the relationship between physical activity (structured) and depressive symptomatology. Lastly, the hypothesis regarding the bi-directional effect between alcohol use (frequency and binge) and depressive symptomatology was only partially supported. What was found was that for both males and females the more alcohol consumed (frequency and binge) the more depressive symptomatology was present. However, a significant negative relationship was found for males and females between depressive symptomatology and frequency of alcohol use. In addition, this same significant negative relationship was found for binge drinking, however only for males. These findings are discussed in detail in the following chapter. 


\section{CHAPTER V. DISCUSSION}

The primary goal of this dissertation was investigate if involvement in physical activity serves as a protective factor against adolescent alcohol use. Furthermore, the current study explored whether depressive symptomatology mediates the relationship between physical activity involvement and adolescent alcohol use. In addition, the current study examined two dimensions of physical activity involvement (i.e. structured sport and unstructured) and adolescent alcohol use (i.e. frequency, and binge). The current study examined these relationships in a sample of male and female adolescents transitioning from middle school to high school. Finally, given extensive literature reviewed in Chapters 1 and 2 pertaining to key gender differences across the key study variables gender was formally examined as a moderator.

This chapter includes a synthesis of study findings. First, results are discussed and summarized within the context of previous studies and current literature. Second, some of the study's strengths and limitations are presented. Finally, conclusions are made regarding the practical implications and the areas of future research.

\section{Synthesis of Findings}

Adolescence is typically a healthy period in life. However, the implications of adolescent health outcomes for overall future health are tremendous due to the initiation of certain health risk behaviors and lifestyle choices that can have enormous impact on current and future health (CDC, 2011). As a result, both the Healthy People 2010 and 2020 Reports established the improvement of healthy development among adolescents as one of their primary goals. In efforts to achieve this goal it is important to understand the health risk behaviors that adolescents engage in and the risk these behaviors can have on 
their overall health. Three out of the six top health goals from Healthy People are to reduce: (1) the proportion of underage persons engaging in current and binge drinking of alcoholic beverages, (2) the proportion of adolescents who report feelings of sadness, unhappiness, or depressed mood, and (3) increase the proportion of adolescents who engage in vigorous physical activity that promotes cardiorespiratory fitness three or more days per week for 20 or more minutes per occasion.

Of particular importance to the current study are the simultaneous increases in the onset of alcohol use and frequency of drinking (e.g., binge drinking) (Johnston, O’Malley, Bachman, \& Schulenberg, 2009), declines in physical activity (CDC, 2008), and increases in depressive symptomatology (SAMSHA, 2008) during middle adolescence. There has been limited exploration of physical activity as a protective factor for risky behaviors such as alcohol use. Largely, what does exist in the literature is inconsistent as it relates to the influence of physical activity on adolescent alcohol use. Some research suggests physical activity is a risk factor for alcohol use (Darling, Caldwell, \& Smith, 2005); while others suggest engaging in physical activity decreases the risk for adolescent drinking (Werch, Moore, DiClemente, Bledsoe, \& Jobli, 2005). This is, in fact, why studying the interplay among these variables during middle adolescence can be particularly compelling. Unfortunately, as discussed in Chapters 1 and 2, research concerning the influence of physical activity on adolescent alcohol use, to this point, has not explored plausible mediators within a longitudinal context to help explain the relationship between these variables. A mediation model was the next logical step to explore the relationship between these variables and to move the field closer to elucidating the underlying mechanisms linking the two variables (physical activity and 
adolescent alcohol use). In particular, the current study looked at depressive symptomatology as a potential mediator in the relationship between physical activity and alcohol use (frequency and binge drinking). In addition, few studies have closely examined whether being involved in organized structured sports differs from unorganized unstructured physical activities in the influence on alcohol-related behaviors.

Thus, the purpose of this dissertation was to place emphasis on the direct influence of physical activity on adolescent alcohol use during middle adolescence, as well as exploring the close associations both variables have with depressive symptomatology. In particular, the current study sought to understand the influence engaging in physical activity (structured and unstructured) during middle adolescence has on alcohol use (frequency and binge), while taking into account depressive symptomatology that so often peaks during this period in life. In addition all of this was examined to assess the extent to which these relationships varied by gender.

Overall, results demonstrate that studying the influence of physical activity (structured and unstructured) may offer critical insight into the relationships among these two forms of physical activity involvement on adolescent alcohol use. However, no significant relation was found between unstructured physical activities and alcohol use (frequency and binge drinking). This lack of a significant relationship may be a function of the innate peer component to structured sports that may not be present in unstructured physical activity involvement; the peer component provides more opportunity to try alcohol.

Findings with regard to binge drinking and its positive associations with structured sport involvement are perhaps the most intriguing and point to a particularly 
important areas for future research. Furthermore, the findings suggest that depressive symptomatology does not mediate the relationship between physical activity (structured and unstructured) and adolescent alcohol use (frequency and binge). This finding is not consistent with past research, which found positive associations between physical activity and depressive symptoms among adolescents (Sanders, 2000; Haugland, Wold, \& Torsheim, 2003; Motl et al., 2004; Allision et al., 2005). In order to establish mediation, a statistically significant relationship had to exist between physical activity (structured and unstructured) and depressive symptomatology at Wave 2. In the current study no significant relationship was found between either structured or unstructured physical activity on adolescent depressive symptomatology in Wave 2. In addition, all hypothesized paths were tested for possible interactions with gender. Gender was found only to interact with depressive symptomatology at Wave 2 and binge drinking.

This study set out to empirically test the influence of structured and unstructured physical activity on alcohol use, as a function of gender, among adolescents transitioning from middle school to high school. Although, contrary to the study hypothesis that physical activity involvement would result in less drinking of alcohol, the fact that a significant inverse relationship between physical activity (structured sport) and binge drinking was present is an indication of how important this line of research is even within a young adolescent population. Contrary to expectations, the results found for structured sports involvement contradict the idea of physical activity serving as a protective factor against alcohol use. In particular, sport involvement positively predicted binge drinking in $9^{\text {th }}$ grade boys and girls. Researchers have called into question the association between sports participation and alcohol use (Sabo et al., 2002). The findings of the current study 
do support previous research (Barber, Eccles, \& Stone, 2001; Mays \& Thompson, 2009) whereby participation in sports is linked to increased alcohol use during the college years (Eccles \& Barber, 1999; Barber et al., 2001). What, in fact, could be taking place is an underlying difference between different types of activities, such as structured activities versus relaxed unstructured activities (Bartko \& Eccles, 2003). Kleiber and colleagues $(1986,1993,1999)$ have placed distinctions among activities that are fun and enjoyable but not demanding and requiring necessary skill development and activities that are thought to require more effort, persistence, and related to skill development, self-concept, and identity. The qualities believed to be involved in the latter more structured activities may be more likely to lead some adolescents to engage in at risk health behaviors in order to fit the attitudes and behaviors of their peers.

In addition, these findings are consistent with research demonstrating that the peer environment surrounding sports participation may encourage drinking behaviors (Barbers et al., 2001; Barbers, Stone, Hunt, \& Eccles, 2005; Lewis, 2008). Prior studies suggest that sports participation may act to increase youths' social status among their peers at school (Eccles et al., 2003), and that during this particular developmental stage, youth are more likely to be exposed to social situations that expose them to alcohol (Windle et al., 2008). Consequently, these adolescents involved in sports could be involved in more social contexts where their peers (e.g., teammates) are using alcohol (e.g., sports-related parties) (Mays et al., 2010).

However, what is important to note is that while researchers have examined the influence of the peer environment on drinking behaviors among intercollegiate athletes (Dams-O'Connor et al., 2007; Lewis, 2008), not much has been done on investigating 
these influences among younger populations such as the sample in this study. This is critical to our advancement in understanding how and when these behaviors manifest themselves and identifying the most optimal times for targeted prevention and interventions (e.g., treatment). Prevention efforts range from limiting young people's access to alcohol to educating them about drinking patterns, outcomes, and coping skills. Whereas, treatment involves tailored intervention approaches for adolescents whose alcohol use behaviors have, in fact, resulted in alcohol use problems and dependence. Furthermore, understanding the impact of other social mechanisms, such as the peer environment, is critical to providing the necessary information of the influence physical activity can have on adolescent alcohol use. Future studies should evaluate the differences between sport involved drinkers and non-drinkers, and sport uninvolved drinkers and non-drinkers. Overall, more research must be conducted to determine if organized youth sports yield a bigger impact on positive health behaviors than less organized physical activity

The relationship between structured physical activity and binge drinking did not hold for frequency of alcohol use. The nature of this relationship warrants further investigation. However, a possible explanation could be seen in the research that has identified social contexts of drinking and peer influences as risk factors for problematic drinking behaviors (e.g., binge drinking) among young people (Ham and Hope, 2003). It is possible that social contexts and peer influences do not hold as strong of an association among adolescents involved in occasional drinking. Social identity comparisons inherent in sport involvement in the context of alcohol use may only function for those involved in binge drinking, which could be why we see this significant relationship for binge 
drinking only. Research has found that infrequent drinkers have a lower personal involvement with alcohol and are less likely to have social interactions with peers that are primarily alcohol related (Yanovitzky, Stewart, \& Lederman, 2006). This could be a unique reflection of those involved in sports having frequent social interactions with peers in the context of alcohol.

Another possible explanation may be similar to what has been seen among the college population. Studies have found that when college-aged/adolescent athletes drink, they most frequently binge drink (Nelson \& Wechsler, 2001; Marten, Dam-O'Connor \& Beck, 2006; Zamboanga \& Ham, 2008). A key factor that could be at play when an adolescent decides to binge drink are alcohol expectancies. The concept of alcohol expectancies provides a theoretical framework for understanding alcohol use and drinking- related behaviors (Goldman, Darkes, \& Del Boca, 1999; Jones, Corbin, \& Fromme, 2001). The current results could in fact be a function of the strong association between structured physical activity involvement (e.g., sports) and binge drinking compared to frequency of alcohol use. This could be in large part what is providing for the significant relationship seen in the present study. As the results demonstrate, there is a difference among the associations between the variables based on frequency of alcohol use and binge drinking. This furthers the notion that these two alcohol drinking behaviors create two distinct groups that warrant further investigation.

The results indicate that the choices adolescents make to be involved in structured versus unstructured physical activities are connected to their alcohol use behaviors. Future studies should seek to better understand alcohol expectancies and their relation to structured and unstructured physical activity involvement. The findings shed light on a 
phenomenon highly researched in college drinking research, but scarcely touched upon in younger populations. Furthermore, involvement in sports takes many forms and given the suggested peer component, future research should examine whether any difference exist among individual sports and team sports as it relates to adolescent alcohol use behaviors.

Another aim of the study was to test, by gender, the bidirectional effect between alcohol use (drinking frequency and frequency of binge drinking) and depressive symptomatology. The reason for including this bidirectional path is because it is quite common to have co-occurrence of alcohol use and depressive symptomatology within the same individual. There has been much research throughout the past two decades indicating that alcohol use is the most common psychoactive substance used, and depression is the most common mental health problem, among adolescents. Several researchers have examined the relationship between adolescent depression and alcohol and tobacco use (Diego et al., 2003; Field et al., 2001). In general, findings suggest a significant relationship between adolescent depression and alcohol use. The direction of the effect is still unclear. Some researchers have hypothesized that depression in adolescents leads to alcohol use, possibly to self-medicate, while others have hypothesized that alcohol use can lead to adolescent depression.

Results from this analysis reveal just how complex this issue is and how challenging it can be to tease the two apart. First, inconsistent with the hypothesis, for both males and females, as depressive symptomatology increased, the frequency of drinking decreased. This was also seen for binge drinking but only for males. Perhaps for males binge drinking takes on such a social component that the more depressed an adolescent male the less likely he is to be interacting with his peers who may also be 
engaging in binge drinking. Also, it is always possible that other substances are being used, which speaks to the limited scope of the current study to only examine alcohol use. Perhaps, the same relationship for binge drinking seen with males happens with females who consume other substances. Other substances need to be explored within the context of the current study to make sure that the same relationship doesn't exist just among a different substance. Second, consistent with the hypothesis, for both males and females, as frequency of alcohol use and frequency of binge drinking increased, depressive symptomatology increased. This is consistent with a large amount of research that has found that adolescents may self-medicate with alcohol in order to alleviate feelings of helplessness (Khantzian, 1997; 2003; Kumpulainen, 2000; Chassin et al., 2002). However, in some cases this only exacerbates the problem, which is why it is so important to continue to devote research efforts into exploring the relationship between alcohol use and depression and other health related behaviors.

To date researchers have been unable to identify which, alcohol or depressive symptomatology, precedes the other and to identify a single track that predicts the course of alcohol use and expression of depressive symptomatology among adolescents. Instead, findings provide strong evidence for variation in drinking patterns and expression of depressive symptomatology among adolescents. Whether depression leads to or is a consequence of alcohol use-remains to be resolved. This could be a result of two distinct pathways; whereby when an adolescent becomes more depressed, they may be less inclined to be involved in social networks where drinking is taking place. On the contrary results demonstrating the more you drink the more depressive symptomatology could be a result of drinking leading to particular physiological and biological reactions (e.g., 
possible neurotransmitters) such as depression. In addition, the more alcohol consumed could result in an adolescent becoming more withdrawn from social and peer networks resulting in more depressive symptomatology. However, in regards to the findings of individuals exhibiting more depressive symptomatology engaging in less alcohol use (frequency of alcohol use and frequency of binge drinking) could be an indication of other mechanisms (e.g., mediators) or moderators having an influence on the relationship that were not examined in this current study. For example, perhaps peers (e.g., friends who drink, access to alcohol etc.) could play a role in the relationship between depressive symptomatology and alcohol use, whereby the more or fewer social networks you have that are engaging in drinking the more likely or less likely you are to drink. Given the fact that there is a social component to drinking if an adolescent is more withdrawn then they may not find themselves in social settings where drinking is occurring. This study serves as a foundation for research efforts examining the complexity of these variables.

It is important to note that this study sample did not include a clinical sample of alcohol dependent adolescents. Perhaps, if a clinical sample were compared with the current sample there would be indications of depressive symptomatology prior to alcohol use. Due to the fact that this comparison was not done, this cannot be ruled out as a possible explanation for the current findings.

Complex behaviors such as drinking patterns and behaviors and depressive symptomatology are the results of biological and physiological mechanism interacting. Therefore, perhaps the best way to continue to approach this line of research is to recognize the unique individuality among adolescents and their reasons for starting to drink and continuing to drink. In addition, there are both implications to prevention and 
intervention/treatment based on these findings which is that universal primary prevention and treatment programs for adolescents that focus on a single health problem are not likely to be successful because adolescents have different problems and the identified risk factors for health behaviors such as alcohol use are necessarily the same for all.

This study also set out to empirically test the mediating effects of depressive symptomatology on the relationship between structured and unstructured physical activity and alcohol use (e.g., frequency and frequency of binge drinking). In contrast to the current study, no published study has looked at a mediational model within a longitudinal design using the same variables. This is one of the reasons why the current study is innovative. Although there is an array of literature pointing to associations between physical activity and depression and between depression and alcohol, depressive symptomatology was not found to be a mediator between physical activity and adolescent alcohol use. Surprisingly, there was no statistically significant relationship between physical activity and depressive symptomatology at Wave 2. Furthermore, there was no statistically significant relationship between depressive symptomatology and adolescent alcohol use (frequency and frequency of binge drinking). However, as mentioned above perhaps different findings would have resulted if the current sample also included clinically diagnosed alcohol dependent adolescents.

This finding could be due in part to the prevalence of depressive symptomatology among the study sample being low. Although, the prevalence of depressive symptomatology is shown to increase during this developmental period (SAMHSA, 2010), perhaps the low incidence of depression observed in this study diminished any associations physical activity would have had on depressive symptomatology and 
depressive symptomatology on alcohol use. This creates a slight restriction in how closely the relationship between physical activity, depressive symptomatology and adolescent alcohol use can be examined. This points to the need for future research to not only look at school samples but clinical samples of depressed or alcohol dependent adolescents. In addition, according to Baron and Kenny (1986), the clearest way to establish mediation is to incorporate three time points. Therefore, it would be interesting to explore and gain a clearer understand and support for mediation by including an additional wave of data.

Despite the fact that depressive symptomatology was not found to be a mediator, results suggest that binge drinking is a mediator between physical activity (structured) and depressive symptomatology for both males and females. An explanation for this finding could be that alcohol use is a precursor to depression. Determining which precedes the other in alcohol and depression research has been a longstanding research challenge. To determine the order of onset more prospective longitudinal studies need to be performed in which otherwise healthy adolescents are followed over a long period of time to ascertain the development of these conditions. What remains clear is that ascertaining which comes first in adolescents is a critical issue for both prevention and treatment efforts. Since, in the current study these two variables were collected at the same time this could have influence the mediation analysis. However, these study findings could provide further support for the research literature that suggest alcohol precedes depression (Rohde, Lewinsohn, \& Seeley, 1996; Rao, Daley, \& Hammen, 2000; Brook, Brook, Zhang, Cohen, \& Whiteman, 2002; Hallfors, Waller, Bauer, Ford, \& Halpern, 2005). The current study also adds new evidence that gender may play a role in 
how these variables play out. Thus, further research investigating meditational pathways is needed.

Furthermore, gender was explored within each aim to test for any possible interactions across study aims by gender. As previously mentioned, this resulted only in a significant interaction between gender and depressive symptomatology at Wave 2 and binge drinking, thus lending some support for possible moderation of paths within the model by gender. These results indicated that for males there was a significant gender interaction between depressive symptomatology at Wave 2 and binge drinking at Wave 2; the more depressed males were, the less frequently they engaged in binge drinking. For females this relationship was not found to be significant. These findings are not consistent with past studies demonstrating that depressive symptomatology results in increased alcohol use (Edwards et al., 2011; Crum et al., 2008; Sihvola et al., 2008). However, the results suggest and provide important evidence that gender may influence the association between depressive symptoms and alcohol use behaviors. Perhaps for males once they express depressive symptomatology they are less inclined to involve themselves in social settings where drinking is taking place.

\section{Strengths of the Study}

This study built on three previous areas of study: (1) physical activity and adolescent alcohol use, (2) physical activity and depression, and (3) depression and alcohol use. To date, such research integrating depression as a potential mediator within studies exploring the influence of physical activity and adolescent alcohol use has not been undertaken. In many ways, the study responded to Healthy People 2010 and 2020's 
objectives of examining the health risk behaviors of adolescents in order to improve the healthy development of adolescents.

The study used data from the National Longitudinal Study of Adolescent Health (Add Health), a dataset that offers a unique opportunity to explore the relationships among these variables over time and within a diverse and nationally representative sample. Although the influence of physical activity on adolescent alcohol use does not have decades of research behind it, evidence is mounting for its importance in the health outcomes of adolescents and adults (Whitt-Glover, Taylor, Floyd, Yore, Yancey, \& Matthews, 2009). The research examining the relationship between physical activity and adolescent alcohol use has found no consistent association between physical activity and alcohol consumption (Sallis et al., 2000). This current study examined depressive symptomatology as a mediator of the relationship between physical activity and adolescent alcohol use. It is one of the few studies that attempted to understand the mechanism by which physical activity influences adolescent alcohol use. In addition, few studies have used longitudinal designs to explore the relationship between physical activity and adolescent alcohol use (Korhanen, Kujala, Rose, \& Kaprio, 2009) Add Health is in many ways well designed for such research, not only because of it longitudinal nature, but also because it is nationally representative data set containing these health related variables of interest.

Another strength of the study was its consideration of gender as a moderator rather than as a predictor in the model. Treating gender as a moderator of the relationships in the models allows for the possibility of identifying differences in the magnitude of the influence of physical activity (structured and unstructured) on 
adolescent alcohol use (frequency and binge) across gender, as well as the process by which depressive symptomatology mediates this relationship. As was found in the current study, only the interaction between gender and depressive symptomatology at Wave 2 resulted in a significant interaction with binge drinking. However, this information could be quite valuable in the development of interventions tailored for how these variables are manifested and interact with one another to create certain health outcomes for males and females.

This study departed from the prevailing approach to studying direct effect models for the influence of physical activity on adolescent alcohol use. This was attempted in efforts to understand the mechanism behind which physical activity may influence adolescent alcohol use. In general, direct effect studies (Barbara, Eccles, \& Stone, 2001; Sabo et al., 2002; Peretti-Watel, Beck, \& Legleye, 2002) have been common, resulting in the development of inconsistent interpretations for the influence of physical activity on adolescent alcohol use. The current study-simultaneously assessed the relationship between physical activity (structured and unstructured) on adolescent alcohol use (frequency and binge) and explored a mechanism by which this relationship may exist. Examining more complex models has been widely recommended (Donovan, 2004) but too infrequently performed. The current study advances the field by ascertaining relationships among these variables and/or constructs that to date have not been studied together.

\section{Limitations of the Study}

Despite the significance and strengths of the study, several limitations to this study should be noted. From a conceptual standpoint, focusing solely on depressive 
symptomatology as a mediator between physical activity (structured and unstructured) and alcohol use outcomes reflects assumptions that this is the only mechanism by which physical activity influences adolescent alcohol use. This assumption would be inaccurate. Other variables and processes most likely also impact and influence the relationship between physical activity and adolescent alcohol use. For example, given the findings of structured physical activity on binge drinking there is reason to believe that a certain peer component of structured physical activity could be in play as it relates to binge drinking. Whether physical activity is a protective or risk factor is still unclear, but what's not unclear is that it is learned from a socialization process with others (Anderssen, 1993). Evidence has been found of an association between sport involvement, peer influences, and substance use behaviors (e.g., Moore \& Werch, 2005; Mays et al., 2010). However, the idea behind the current study was that no study to date had examined depressive symptomatology as a plausible mediator in the relationship among physical activity and adolescent alcohol. Moreover, given the extensive amount of research examining the associations among these variables it seemed fitting to begin some exploration in this area whereby future research could expound upon.

As mentioned earlier in the synthesis of the findings, the non-inclusion of a clinical sample of alcohol dependents and those individuals diagnosed with clinical depression could have impacted the findings for the current study. This limited the studies ability to expand the findings to include both a nationally representative schoolbased sample, as well as, as a clinically diagnosed sample. Among other things, not including other substances may have diminished the findings by limiting the possible relationships physical activity and depressive symptomatology have with other 
substances of choice by adolescents. It is possible that results may have differed if other drugs and clinical populations had been included within the analysis.

Several methodological limitations were also present in the current study. Of particular importance is that the study model may be incomplete. Other variables and processes most likely also influence the relationship between physical activity and alcohol use drinking behaviors. The current study relied exclusively on self-report measures, which could possibly have led to biased reporting and measurement issues related to the internal validity of the results. Although the questionnaire method is one of the most feasible options when dealing with such a large population study of this caliber, measurement error is the most serious shortcoming. In particular, under- or over-report may contaminate the results of this study.

Furthermore, inherent in any study using a dataset not specifically designed for the purposes of the study result in some limitations. In large part these limitations involve the availability of the most appropriate measures to capture the variables to be studied and operationalization of the measures present in the current study. For example, the most important limitation pertaining to the measures was the measure to assess physical activity in the Add Health data set. This questionnaire only included three questions. A standardized physical activity measure was not used in the collection of information pertaining to physical activity involvement among study participants. Therefore, the reliability and validity of the subscales are unknown. Although it is plausible that some of the differences in results could be a result of differences in how these behaviors are operationalized and measured, this particular study utilizes physical activity behaviors that act as a reasonable proxy measure of physical activity involvement. However, future 
studies should include further questions or a standardized physical activity questionnaire or diary to aid in confirming and strengthening the physical activity measure as well as future study findings. In addition, the dependent variable and mediator in this study were collected at the same time point (Wave 2), interfering with the ability to establish causality in the mediation analysis.

Software limitations presented another set of challenges for this study. Mplus is one of only three software programs available for testing structural equation modeling with complex survey data. This hinders the ability to engage in multiple group analysis, which would have allowed for a better test of the moderation by gender by identifying difference in levels of effect across gender. As a result, the conclusions drawn from the analysis are tentative in a SEM context. Future research should make sure to address the limitations of past research efforts in order to be more inclusive and provide even more methodologically sound and innovative approaches to exploring the influence of physical activity on adolescent alcohol use.

\section{Summary}

In sum, the influence of physical activity on adolescent alcohol use remains complex. However, there appears to be a significant inverse relationship present regarding the influence of structured physical activity (e.g., sports) on binge drinking behaviors during middle adolescence. This finding has very serious implications. In order for prevention and intervention programs to be effective, there needs to be a better understanding of what makes certain forms of physical activity more negatively associated with adolescent alcohol use behaviors. Current research overwhelmingly has looked at the association of sport involvement and binge drinking among collegiate 
students (Marten, Dam-O'Connor \& Beck, 2006; Zamboanga \& Ham, 2008). However, it can be argued from the findings in the present study that there is a pressing need to evaluate these relationships at a much younger age. Given the serious short and long-term negative consequences of adolescent alcohol use, it is imperative that adolescents receive the prevention, services, and treatment efforts they need regarding alcohol use drinking behaviors, physical activity involvement and depressive symptomatology, which this study demonstrates are all associated with one another.

It is evident that improvements in adolescent health require interdisciplinary, collaborative, and comprehensive efforts. The involvement of social work, with this discipline's expertise and broad scope of involvement with adolescents and their families, schools, and health service institutions, is vital to the achievement of good health outcomes. Each social system has a potential impact on the adolescent, and the active involvement of all systems is necessary to fully maximize existing resources to prevent underage drinking and its related problems (USDHHS, 2007). The social work profession values the fundamental underlying need for positive adolescent development as an effective approach in improving adolescent health. Social work has long acknowledged the need for a "new paradigm" in defining and addressing adolescent health by highlighting strengths and assets on which to build and that time has come. The following two sections will discuss the implications of the study findings to social work practice and policy.

\section{Implications for Social Work Research, Practice and Policy}

Despite the limitations addressed earlier, the results of this study suggest several important implications for social work research, practice, and policy. Likewise, the 
solution to the problem of underage drinking is complex and must involve addressing many pathways simultaneously (e.g., biological, psychological, and social). One of the first and most important changes that can be made in alcohol use prevention strategies is to broaden them beyond an exclusive focus on late adolescence, early adulthood, and adulthood. As the results from this study demonstrate, adolescents in the $8^{\text {th }}$ and $9^{\text {th }}$ grade are binge drinking and there appears to be a significant positive relationship between binge drinking and sport involvement. This is quite similar to what is seen in the literature for the college age population. Therefore, it is imperative to reach our earlier aged populations when it comes to intervention efforts addressing alcohol use. The primary implication for social work research is to bring a person-in-the-environment perspective to explore the culturally diverse manifestations of adolescent alcohol use. This includes social/interpersonal risk factors such as peers, as well as, contextual/cultural risk factors such as individual and macro level systems. Knowing about the wide array of influences and mechanisms of operation facilitate both prevention and treatment.

Although depressive symptomatology was not found to be a mediator in the relationship between physical activity and adolescent alcohol use, binge drinking was found to be mediator. The results show that adolescents participating in structured physical activities present complex treatment challenges including both the binge drinking and resulting high rates of depressive symptomatology. This finding speaks to how important multidisciplinary and comprehensive approaches are to addressing adolescent alcohol use problems. From the interpretation of findings, several implications 
are identified and recommendations are made considering further research, practice, and policy development.

Research. Research can help a great deal in determining the most effective policies and prevention approaches in responding to the problem of underage drinking. Fincham (1999) proposed that research involves three stages: (1) the association of risk factors to outcomes, (2) the identification of mechanisms (mediators) that account for the associations, and (3) the development of interventions that modify the associations between risk and outcome. In the field of adolescent alcohol use stage one has received considerable attention. The same cannot be said for stage two.

Furthermore, stage three is performed sometimes prematurely without sufficient attention to stage two. The current study addressed some of the deficiencies in stage two by examining a potential mediator (e.g., depressive symptomatology) in the relationship between physical activity and adolescent alcohol use. Although, depression was not found to mediate the relationship, it was an attempt at including more stage two research. Instead, binge drinking was found to mediate the relationship between physical activity (sports) and depressive symptomatology at Wave 2. This finding prompts intervention research to look at the influence of sport involvement on binge drinking and resulting mental health problems (e.g., depression).

The greatest influence on young people's decisions to begin drinking is the world they live in, which includes their families, friends, schools, the larger community, and society as a whole. Proposed changes around the future of research should include closer examination into the individual, family, social, and environmental level influences on adolescent alcohol use. There is a definite peer component to being involved in structured 
physical activities (e.g., sports), which could explain the significant association found between sport involvement and binge drinking in the current study. For this reason, it is imperative to place more research efforts in community-based participatory research, as well as, evidenced-based research practices. Also, more research should examine the effect of cognitive alcohol expectancies among adolescent populations to see what influence it has on alcohol use behaviors. For example, given some of the discussion of the current study's findings, person-centered approaches with longitudinal data could be utilized to explore more fully the associations among sport and non-sport involvement and adolescent alcohol use behaviors. It is also important to point out that because this sample was a school-based sample, the association among these variables among clinical samples deserves further attention. Knowledge from this line of research could lead to the targeted design of more beneficial prevention and intervention efforts to address alcohol initiation and drinking behaviors.

Practice. This study has several implications for interventions related to health behaviors. The findings from this research have strong implications for social workers, public health professionals, and other health educators to initiate implementation of prevention programs as early as elementary school and continue through middle and high school. It is imperative for social workers to become knowledgeable on the continuum of the manifestation of alcohol use from childhood to adulthood. This ensures early efforts to help prevent and/or eliminate the initiation of this behavior and the resulting negative health consequences. The findings suggest the prevention, assessment, and treatment of adolescent alcohol use should be multi-dimensional (Werch et al., 2005). 
On an individual level youth need to not only understand or view alcohol consumption from a legal perspective but also to develop more responsible beliefs and attitudes around alcohol consumption. During adolescence, it is important to understand how peers can affect health behaviors. Therefore, not only should adolescent alcohol use be prevented, assessed, and treated, but the influence of peers, in particular how one compares and identifies themselves within a social network, should be considered. In order to effectively intervene, it will not be sufficient to focus only on the adolescent. As Werch and colleagues (2005) have noted, multiple strategies should be employed by researcher and practitioners to address adolescent alcohol use.

Furthermore, the current study suggests one particular contextual variable (e.g., peers) that may be influential in the relationship between physical activity and adolescent alcohol use and should be included in prevention and intervention programs implemented by social workers. Social workers can discuss with adolescents what they are hearing from their peers as it relates to alcohol use. Also, social workers can possibly transmit the message across more adolescents, thereby increasing the likelihood of touching more and more adolescents. The findings from this study as it relates to sport involvement suggest that assessment and intervention practices may need to be modified to include assessing the whole teams alcohol use behaviors. The social bonds created amongst teammates can potentially foster positive socialization environments where alcohol use is discouraged or implications in the consumption of alcohol.

The findings from this study suggest that pro-alcohol peer exposure from sports and social situations increases the risk of underage drinking. As part of an intervention, on a group level, social workers could conduct groups with sport teams that help 
adolescents explore the positive advantages of being involved in sports and staying away from alcohol. For example, the importance of avoiding alcohol for overall health and athletic performance can be emphasized. Teaching adolescents how to be involved in social situations and peer environments that are not defined by alcohol can be a part of this therapeutic process. Since sports participation was related to binge drinking, the social work practitioner can also work to encourage alcohol prevention across multiple settings. These settings could include not only the team, but at home with the parents, the school, and the coaches. Practitioners could encourage parental and coach involvement in team social events; which could help to reduce the likelihood of exposure, availability and consumption of alcohol. In the school, the social work practitioner can implement targeted programs for athletes to encourage abstinence from alcohol. These programs could include teacher involvement and training and consistent consequences for athletes who are caught drinking.

Schools have increasingly become the place where adolescents interact with, not only their peers, but adults. Teachers, administrators, and school social workers should work closely together in promoting the health-related benefits of exercise and how sports and alcohol use do not go together. Teachers and school social workers could provide during and after school activities involving substance use prevention programs in conjunction with sports involvement.

On a community level, social workers can advocate for increased advertising of the benefits of exercise and the risks of alcohol use by actively collaborating with other professional, community organizations, and associations. For example, school and coaches with the help from social workers can target social service agencies that work 
closely with adolescent at risk for alcohol use and misuse. Once this connection is made social workers can help to foster the relationships among school, coaches and social service agencies to work closely in identifying adolescents on sport teams who appear to be at risk for alcohol use. The more collaboration and involvement across the adolescents social network the higher the likelihood to create a continual and constant message of alcohol prevention.

Policy. Current policy surrounding underage drinking will inevitable prevent some youth from initiation of alcohol use before the age of 21 . However, some will choose to engage in alcohol consumption despite the law. Perhaps, the current approaches have accomplished what they can and it is now time to move into a new era of investigation and develop new attitudes, beliefs, cultures, skills, and strategies around current policy to address the issue. The hope in the current study was to find involvement in physical activities as a protective factor. However, the opposite was found to be the case as it relates to structured sport involvement. Perhaps with further research on the influence of peer drinking behaviors associated with sport involvement, it is possible to gain a clearer understanding of the diverse mechanisms affecting the influence of structured physical activity on binge drinking. Then interventions for adolescent alcohol use should include specific components to address specific risk factors that sports involvement has on alcohol use behaviors. If and when these interventions can demonstrate effectiveness, then it justifies devoting resources at the local, state, and federal level to implement programs to prevent or reduce alcohol use among this highrisk group. Social workers are highly qualified to work across all three levels to promote 
funding for these interventions and playing a role implementing overall health behavior change.

Social workers have often been at the forefront of substance abuse treatment and prevention efforts among adolescent and adult populations (Bliss, 2007). In the adolescent population social workers have played a key role in the development and implementation of prevention and treatment programs to reduce underage drinking. While social work involvement in treating and preventing alcohol use has been significant their involvement in conducting research that leads to effective evidenced based interventions has been limited (Segal, Gerdes, \& Steiner, 2009). However, in recent years social workers have become more involved in conducting research into effective treatments in dealing with the problem of alcohol use, abuse, and dependency (Segal, Gerdes, \& Steiner, 2009). For example, several studies in social work have examined comorbidity, the presence of interconnected substance abuse and mental illness and/or physical illness, health disparities and substance abuse, as well as the impact of substance abuse on families. In addition, during the past twenty years there has been an emphasis by the social work profession to contribute additional knowledge to addressing alcohol use as evidenced by the professions efforts to involve itself more deeply in researching the underlying factors responsible for this problem among our Nation's adolescents (Straussner, 2001).

As a result, social workers are truly in a position to influence the research and the delivery of services to address the acute and chronic needs of vulnerable populations and high-risk individuals for alcohol use and alcohol related problems. Through the continued development, application, and incorporation of evidence-based approaches in 
interventions based off of cutting edge research, social workers can significantly impact treatment and prevention service for delivery for clients and their families (National Association of Social Work, 2005). Furthermore, social workers should not only provide resources to community services but should advocate on behalf of adolescents during political issues to promote social justice. In summary, future recommendations proposed above are a comprehensive strategy and an ambitious endeavor that, taken as a whole, would foster a deep unequivocal societal commitment to curtail underage drinking.

\section{Conclusions and Future Directions}

Without a doubt, adolescent alcohol use is a difficult behavior to change because alcohol use has been woven into so many facets of U.S. culture. Much remains to be learned regarding the etiology, influences, associations, and development of alcohol problem among adolescents and the impact of different strategies for their treatment and prevention. There is a pressing need for research and programmatic agendas concerning these issues to implement multidisciplinary and comprehensive approaches to addressing the problem.

Although this dissertation's findings are promising, further research is needed to refine the tested model. From the results of the current study, several recommendations for future studies and ways of refining the current study model can be suggested. For example, additional research is needed to better understand the mechanisms by which sports participation is associated with binge drinking behaviors among middle adolescents. An approach could be to explore non-drinking adolescents involved in sports compared to drinking adolescents involved in sports to research the plausible norms for drinking among this population. In addition, perhaps to tease apart the peer component, 
future research should examine whether there is any differentiation between individual versus team sports. Another avenue for future research would be to consider specific expectancy domains (e.g., cognitive and behavioral) to determine if there are content areas that deserve attention. Overall, continued research is needed to better understand the relationship between physical activity and adolescent alcohol use.

The model evaluated in this dissertation explicitly recognizes the potential importance of possible protective factors and mediators (e.g., depressive symptomatology) in predicting adolescent alcohol use behaviors. Another research area of key interest is how alcohol and depressive symptomatology are associated. It has been proven to be a challenge to elucidate which precedes the other. In this study significant reciprocal relationships were found between alcohol use (frequency and binge drinking) and depression. This speaks to the need for further research to tease apart these two conceptualizations of alcohol use behaviors. In addition, as mentioned in the review of the literature there does exist some degree of variability across race/ethnicity within the study variables. Although, this was not explored as an aim of the current study it is imperative that this be explored in future research. Given, the very key discrepancies across the mediator (depressive symptomatology), it was extremely fundamental and important to include gender analysis into the main aims of the study.

In regards to the analysis of bidirectionality, future research should examine possible other variables that could explain the inverse relationship found between depressive symptomatology and adolescent alcohol use (frequency of alcohol use and binge drinking). For example, the variable of friends who drink, used as an instrumental variable in the current study could perhaps be examined as a moderator or mediator of the 
relationship between depressive symptomatology and adolescent alcohol use. So future research should closely examine other variables that could be interacting with this relationship to help in providing some explanation for the relationship found in the current study. In addition, the current study served as a foundation to assess whether the relationships proposed hold. However, future research should include additional waves and examine cross-lagged effects to really assess bidirectionality. If the cross-lagged paths are both significant than it is possible to say with more certainty that there is bidirectionality.

However, what the results do offer is a justification for researchers to examine beyond direct effect models. The continued expansion and examination of this study model is needed to further determine the relationship between physical activity and adolescent alcohol use. The current study serves as a foundation for the development of more complex and inclusive models. The findings serve as a building block for future phenomenological research that will consider broader populations of middle school-aged and high school-aged students identified to lack physical activity involvement and drinkers and non-drinkers. Clearly, there is an association between structured physical activity (e.g., sport) and binge drinking which requires additional attention. Finally, the role of peer alcohol use and the perception of peer alcohol use warrant further investigation given the influential factor it is believed to have with sport involvement. 
Table 1

Demographic Information Reported in Frequencies, Standard Deviations and Errors for Add Health Study Participants

Frequencies $^{\mathrm{a}}(S D)$ and $(S E)$

\begin{tabular}{|c|c|c|}
\hline Variable & Unweighted $^{1}$ & Weighted $^{2}$ \\
\hline Age & $14.02(.68)$ & $14.05(.02)$ \\
\hline
\end{tabular}

Gender

Males

48.9

$51.9(1.1)$

Females

51.1

$48.1(1.1)$

Race/Ethnicity

White

58.2

24.0

11.7

$67.9(4.4)$

Black

6.1

$15.8(3.3)$

Hispanic

$11.3(2.4)$

Other $^{\mathrm{b}}$

$34.45(23.35)$

$5.0(1.0)$

Parental Income

$33.73(1.57)$

Parental Education

Less than $8^{\text {th }}$ grade

4.1

$\begin{array}{ll}\text { Did not graduate high school } & 10.8 \\ \text { High school degree } & 29.3\end{array}$

$.3(.2)$

High school degree

29.3

$4.1(.9)$

Trade school

16.2

$12.6(1.7)$

College but no degree

18.2

College degree

13.1

$31.6(1.7)$

$15.5(1.3)$

Professional beyond college

8.4

$17.7(1.2)$

$6.7(.9)$

Note. ${ }^{\mathrm{a}} N=2054 ;{ }^{1}$ SDs were reported for unweighted data where applicable; ${ }^{2}$ SEs were reported for weighted national representation, and SEs were corrected for multiple stages of cluster sample design and unequal probability of selection; ${ }^{b}$ Other in the Race/Ethnicity category represents Asian and Native Americans 
Table 2

Means, Standard Deviations and Errors of Study Variables both Unweighted and Weighted for Total Sample and by Gender

\begin{tabular}{|c|c|c|c|c|c|c|}
\hline \multirow[b]{2}{*}{ Variables } & \multicolumn{2}{|c|}{ Sample $^{\mathrm{a}}$} & \multicolumn{2}{|c|}{ Males $^{\mathrm{b}}$} & \multicolumn{2}{|c|}{ Females $^{c}$} \\
\hline & Unweighted & Weighted $^{2}$ & Unweighted & Weighted $^{2}$ & Unweighted & Weighted $^{2}$ \\
\hline $\begin{array}{l}\text { Level of physical activity } \\
\text { participation in } \\
\text { sports/week at wave } 1 \\
\text { (PA_Sport_1) }\end{array}$ & $1.63(1.12)$ & $1.62(.04)$ & $1.92(1.09)$ & $1.84(.05)$ & $1.35(1.10)$ & $1.39(.05)$ \\
\hline $\begin{array}{l}\text { Level of participation in } \\
\text { unstructured } \\
\text { activities/week at wave } 1 \\
\text { (PA_US_1) }\end{array}$ & $1.31(.75)$ & $1.33(.02)$ & $1.41(.80)$ & $1.442(.04)$ & $1.21(.69)$ & $1.23(.03)$ \\
\hline $\begin{array}{l}\text { Level of depressive } \\
\text { symptoms (CESD total } \\
\text { score) wave } 2 \\
\text { (CESD_2) }\end{array}$ & $10.70(7.51)$ & $10.60(.24)$ & $9.45(6.50)$ & $9.44(.25)$ & $11.89(8.20)$ & $11.86(.35)$ \\
\hline $\begin{array}{l}\text { Frequency of Binge } \\
\text { drinking at wave } 2 \\
\text { (Binge_2) }\end{array}$ & $1.40(1.70)$ & $1.65(.05)$ & $1.65(1.87)$ & $1.62(.14)$ & $1.20(1.51)$ & $1.13(.08)$ \\
\hline $\begin{array}{l}\text { Frequency of alcohol use } \\
\text { at wave } 2 \text { (FAU_2) } \\
\text { Covariates }\end{array}$ & $2.05(1.46)$ & $2.31(.04)$ & $2.21(1.54)$ & $2.20(.10)$ & $1.91(1.37)$ & $1.94(.09)$ \\
\hline $\begin{array}{l}\text { Level of depressive } \\
\text { symptoms (CESD total } \\
\text { score) wave } 1 \\
\text { (CESD_1) }\end{array}$ & $10.28(7.35)$ & $10.10(.24)$ & $9.29(6.48)$ & $9.39(.30)$ & $11.22(7.99)$ & $10.87(.34)$ \\
\hline $\begin{array}{l}\text { Level of Binge drinking at } \\
\text { wave } 1 \text { (Binge_1) }\end{array}$ & $1.11(1.59)$ & $1.16(.08)$ & $1.33(1.79)$ & $1.37(.13)$ & $.91(1.37)$ & $.97(.10)$ \\
\hline $\begin{array}{l}\text { Level of Frequency of } \\
\text { alcohol use at wave } 1 \\
\text { (FAU_1) } \\
\text { Instrumental Variable }\end{array}$ & $1.56(1.43)$ & $1.56(.06)$ & $1.61(1.56)$ & $1.59(.10)$ & $1.52(1.30)$ & $1.53(.07)$ \\
\hline $\begin{array}{l}\text { Number of Friends who } \\
\text { drink at wave } 2 \\
\text { (FrWhoD_2) }\end{array}$ & $.95(1.096)$ & $1.04(.04)$ & $.95(1.11)$ & $1.05(.05)$ & $.96(1.09)$ & $1.04(.05)$ \\
\hline
\end{tabular}

Note. ${ }^{\mathrm{a}} N=2054,{ }^{\mathrm{b}} n=1004,{ }^{\mathrm{c}} n=1050 ;{ }^{1}$ SDs were reported for unweighted data; ${ }^{2} \mathrm{SEs}$ were reported for weighted national representation, and SEs were corrected for multiple stages of cluster sample design and unequal probability of selection; CES-D = Center for Epidemiologic Studies Depression Scale; Number of Friends who drink at wave $2=$ instrumental variable used in bidirectional analyses 
Table 3

Skewness and Kurtosis for Study Variables

\begin{tabular}{lcc} 
Variable & Skewness & Kurtosis \\
\hline $\begin{array}{l}\text { Level of physical activity participation in } \\
\text { sports/week at Wave 1 }\end{array}$ & -.09 & -1.39 \\
$\begin{array}{l}\text { Level of participation in unstructured } \\
\text { activities/week at Wave 1 }\end{array}$ & .34 & -.35 \\
$\begin{array}{l}\text { Level of depressive symptoms (CESD } \\
\text { total score) Wave 2 }\end{array}$ & 1.13 & 1.59 \\
$\begin{array}{l}\text { Frequency of Binge drinking at Wave 2 } \\
\text { Frequency of alcohol use at Wave 2 }\end{array}$ & 1.16 & .32 \\
$\begin{array}{l}\text { Covariates } \\
\text { Level of depressive symptoms (CESD } \\
\text { total score) Wave 1 }\end{array}$ & .77 & -.10 \\
$\begin{array}{l}\text { Level of Binge drinking at Wave 1 } \\
\text { Level of Frequency of alcohol use at wave } \\
\text { 1 (FAU_1) }\end{array}$ & 1.15 & 1.69 \\
$\begin{array}{l}\text { Instrumental Variable } \\
\text { Number of Friends who drink at wave 2 } \\
\text { (FrWhoD_2) }\end{array}$ & & \\
\hline
\end{tabular}

Note. Number of Friends who drink at wave $2=$ instrumental variable used in bidirectional analyses 
Table 4

Path Coefficients, $p$ values and 95\% Confidence Intervals for SEM Model with Frequency as Outcome for Males

$95 \%$ C. I.

Frequency of Alcohol Use Wave 2 ON

Level of physical activity participation in

Level of physical activity in participation in

unstructured activities

$0.02 \quad 0.72 \quad-0.08$

0.11

Level of depressive symptoms Wave 1

$\begin{array}{llll}0.11 & 0.24 & -0.08 & 0.30\end{array}$

Frequency of alcohol use Wave 1

$0.02 \quad 0.14$

$-0.01$

0.05

Dsex

0.28

0.00

0.19

0.38

Black

0.05

0.74

$-0.22$

0.31

Hispanic

$-0.10$

0.46

$-0.37$

0.17

Other

$-0.13$

0.39

$-0.42$

0.16

Education

0.04

0.86

$-0.37$

0.44

Income

$-0.05$

0.23

$-0.12$

0.03

Age

0.00

0.70

$-0.01$

0.00

Friends who drink Wave 2

0.21

0.05

0.00

0.42

0.47

0.00

0.37

0.57

Level of depressive symptoms Wave 2

$-0.10$

0.02

$-0.19$

$-0.01$

DSex_Depressive symptoms

0.11

0.01

0.03

0.19

Level of depressive symptoms Wave2 ON

Level of physical activity participation in sports

$-0.23$

0.17

$-0.56$

0.10

Level of physical activity in participation in unstructured activities

$\begin{array}{llll}-0.03 & 0.91 & -0.54 & 0.48\end{array}$

Level of depressive symptoms Wave 1

0.53

0.00

0.46

0.60

Frequency of alcohol use Wave 1

$-0.30$

0.32

$-0.90$

0.29

Dsex

$\begin{array}{ll}1.85 & 0.00\end{array}$

1.16

2.53

Black

1.03

0.03

0.12

1.94

Hispanic

0.89

0.17

$-0.39$

2.17

Other

1.01

$-0.38$

2.39

Education

$-0.04$

0.16

$-0.28$

0.20

Income

$-0.01$

0.74

$-0.02$

0.01

Age

0.36

0.19

$-0.27$

0.99

Frequency of alcohol use Wave 2

$\begin{array}{ll}1.36 & 0.00\end{array}$

0.46

2.26

Notes. B signifies unstandardized path coefficients; $95 \% \mathrm{CI}=95 \%$ confidence interval for unstandardized path coefficient; ${ }^{b}$ signifies that the interaction contrast was significant. 
Table 5

Path Coefficients, $p$ values and 95\% Confidence Intervals for SEM Model with Frequency as Outcome for Females

\begin{tabular}{|c|c|c|c|c|}
\hline \multirow[b]{2}{*}{ Frequency of Alcohol Use Wave 2 ON } & \multirow[b]{2}{*}{$\beta$} & \multirow[b]{2}{*}{$p$-value } & \multicolumn{2}{|c|}{$95 \%$ C. I. } \\
\hline & & & Lower 2.5 & pper $2.5 \%$ \\
\hline $\begin{array}{l}\text { Level of physical activity participation in } \\
\text { sports }\end{array}$ & 0.04 & 0.37 & -0.05 & 0.13 \\
\hline $\begin{array}{l}\text { Level of physical activity in participation in } \\
\text { unstructured activities }\end{array}$ & 0.12 & 0.23 & -0.07 & 0.31 \\
\hline Level of depressive symptoms Wave 1 & 0.02 & 0.12 & -0.01 & 0.04 \\
\hline Frequency of alcohol use Wave 1 & 0.29 & 0.00 & 0.20 & 0.38 \\
\hline Dsex & 0.08 & 0.46 & -0.13 & 0.29 \\
\hline Black & -0.16 & 0.21 & -0.42 & 0.09 \\
\hline Hispanic & -0.18 & 0.18 & -0.45 & 0.09 \\
\hline Other & -0.02 & 0.90 & -0.35 & 0.31 \\
\hline Education & -0.04 & 0.28 & -0.11 & 0.03 \\
\hline Income & 0.00 & 0.55 & -0.01 & 0.00 \\
\hline Age & 0.17 & 0.10 & -0.03 & 0.38 \\
\hline Friends who drink Wave 2 & 0.45 & 0.00 & 0.35 & 0.55 \\
\hline Level of depressive symptoms Wave 2 & -0.06 & 0.04 & -0.12 & 0.00 \\
\hline DSex_Depressive symptoms & 0.06 & 0.01 & 0.01 & 0.10 \\
\hline Level of depressive symptoms Wave 2 ON & & & & \\
\hline $\begin{array}{l}\text { Level of physical activity participation in } \\
\text { sports }\end{array}$ & -0.23 & 0.17 & -0.56 & 0.10 \\
\hline $\begin{array}{l}\text { Level of physical activity in participation in } \\
\text { unstructured activities }\end{array}$ & -0.03 & 0.91 & -0.54 & 0.48 \\
\hline Level of depressive symptoms Wave 1 & 0.53 & 0.00 & 0.46 & 0.60 \\
\hline Frequency of alcohol use Wave 1 & -0.30 & 0.32 & -0.90 & 0.29 \\
\hline Dsex & -1.85 & 0.00 & -2.53 & -1.16 \\
\hline Black & 1.03 & 0.03 & 0.12 & 1.94 \\
\hline Hispanic & 0.89 & 0.17 & -0.39 & 2.17 \\
\hline Other & 1.01 & 0.16 & -0.38 & 2.39 \\
\hline Education & -0.04 & 0.74 & -0.28 & 0.20 \\
\hline Income & -0.01 & 0.19 & -0.02 & 0.01 \\
\hline Age & 0.36 & 0.26 & -0.27 & 0.99 \\
\hline Frequency of alcohol use Wave 2 & 1.36 & 0.00 & 0.46 & 2.26 \\
\hline
\end{tabular}

Notes. B signifies unstandardized path coefficients; $95 \% \mathrm{CI}=95 \%$ confidence interval for unstandardized path coefficient; ${ }^{b}$ signifies that the interaction contrast was significant. 
Table 6

Path Coefficients, $p$ values and 95\% Confidence Intervals for SEM Model with Binge Drinking as Outcome for Males

95\% C. I.

\begin{tabular}{|c|c|c|c|c|}
\hline \multirow[b]{2}{*}{ Frequency of binge drinking Wave 2 ON } & \multirow[b]{2}{*}{$\beta$} & \multirow[b]{2}{*}{$p$-value } & \multicolumn{2}{|c|}{$95 \%$ C. I. } \\
\hline & & & Lower 2. & pper $2.5 \%$ \\
\hline $\begin{array}{l}\text { Level of physical activity participation in } \\
\text { sports }\end{array}$ & 0.11 & 0.05 & 0.00 & 0.21 \\
\hline $\begin{array}{l}\text { Level of physical activity in participation in } \\
\text { unstructured activities }\end{array}$ & 0.15 & 0.21 & -0.08 & 0.38 \\
\hline Level of depressive symptoms Wave 1 & 0.04 & 0.04 & 0.00 & 0.07 \\
\hline Frequency of binge drinking Wave 1 & 0.41 & 0.00 & 0.31 & 0.51 \\
\hline Dsex & -0.05 & 0.69 & -0.31 & 0.20 \\
\hline Black & -0.54 & 0.04 & -1.06 & -0.02 \\
\hline Hispanic & 0.35 & 0.08 & -0.04 & 0.73 \\
\hline Other & -0.05 & 0.82 & -0.51 & 0.40 \\
\hline Education & -0.02 & 0.71 & -0.13 & 0.09 \\
\hline Income & -0.01 & 0.14 & -0.01 & 0.00 \\
\hline Age & 0.18 & 0.10 & -0.03 & 0.40 \\
\hline Friends who drink Wave 2 & 0.48 & 0.00 & 0.35 & 0.60 \\
\hline Level of depressive symptoms Wave 2 & -0.12 & 0.03 & -0.22 & -0.01 \\
\hline DSex_Depressive symptoms & 0.12 & 0.01 & 0.04 & 0.20 \\
\hline Level of depressive symptoms Wave 2 ON & & & & \\
\hline $\begin{array}{l}\text { Level of physical activity participation in } \\
\text { sports }\end{array}$ & -0.35 & 0.10 & -0.76 & 0.07 \\
\hline $\begin{array}{l}\text { Level of physical activity participation in } \\
\text { unstructured activities }\end{array}$ & -0.04 & 0.87 & -0.54 & 0.45 \\
\hline Level of depressive symptoms Wave 1 & 0.50 & 0.00 & 0.42 & 0.58 \\
\hline Frequency of binge drinking Wave 1 & -0.32 & 0.23 & -0.84 & 0.20 \\
\hline Dsex & 2.11 & 0.00 & 1.37 & 2.84 \\
\hline Black & 1.53 & 0.00 & 0.51 & 2.55 \\
\hline Hispanic & 0.34 & 0.56 & -0.81 & 1.48 \\
\hline Other & 1.25 & 0.10 & -0.23 & 2.74 \\
\hline Education & -0.05 & 0.73 & -0.31 & 0.22 \\
\hline Income & 0.00 & 0.74 & -0.02 & 0.02 \\
\hline Age & 0.38 & 0.18 & -0.17 & 0.94 \\
\hline Frequency of binge drinking Wave 2 & 1.33 & 0.00 & 0.52 & 2.13 \\
\hline
\end{tabular}

Notes. B signifies unstandardized path coefficients; $95 \% \mathrm{CI}=95 \%$ confidence interval for unstandardized path coefficient; ${ }^{b}$ signifies that the interaction contrast was significant. 
Table 7. Path Coefficients, $p$ values and 95\% Confidence Intervals for SEM Model with Binge Drinking as Outcome for Females

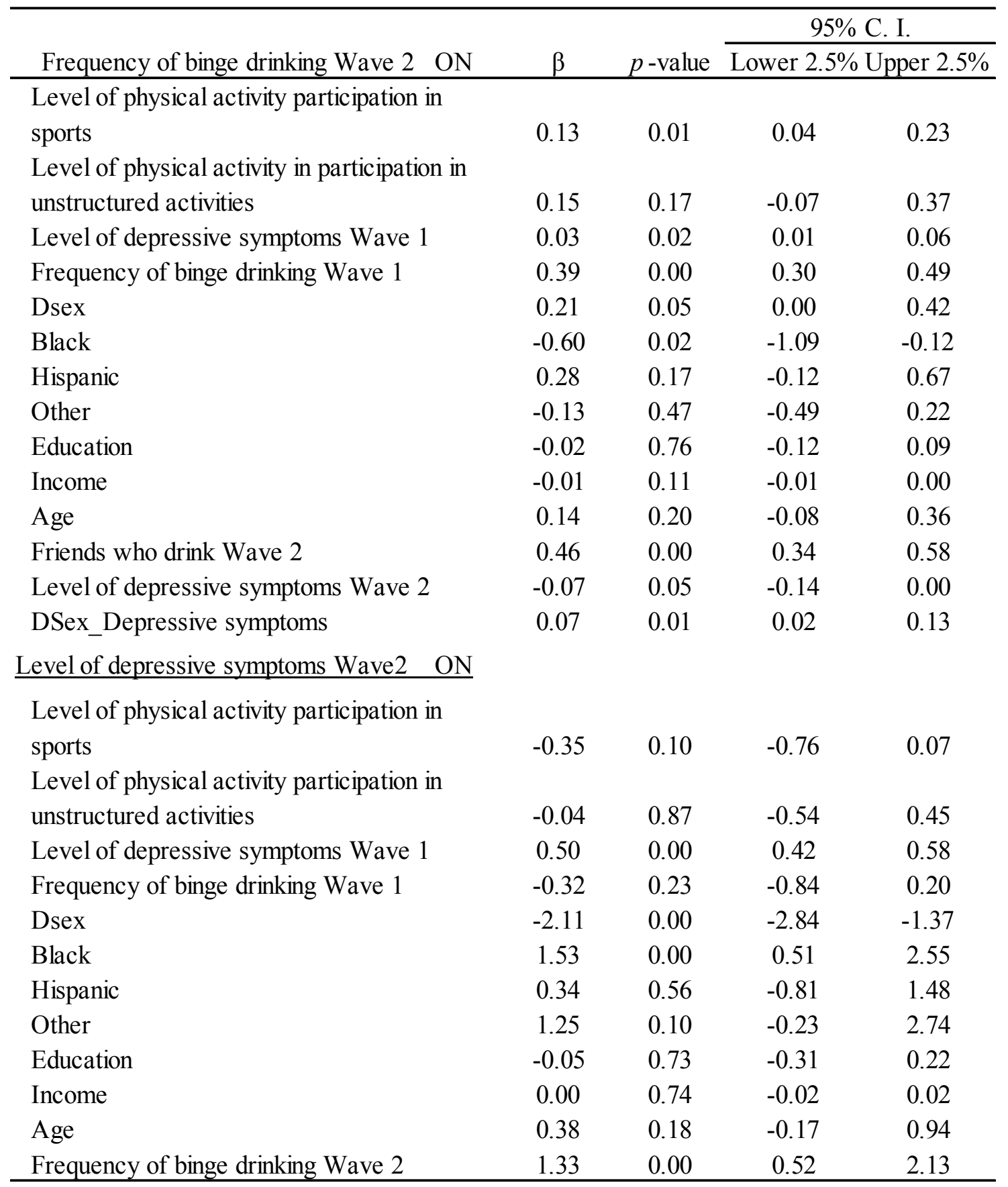

Notes. B signifies unstandardized path coefficients; $95 \% \mathrm{CI}=95 \%$ confidence interval for unstandardized path coefficient; ${ }^{b}$ signifies that the interaction contrast was significant. 
Figure 1. Conceptual Model with Frequency of Alcohol Use as Outcome

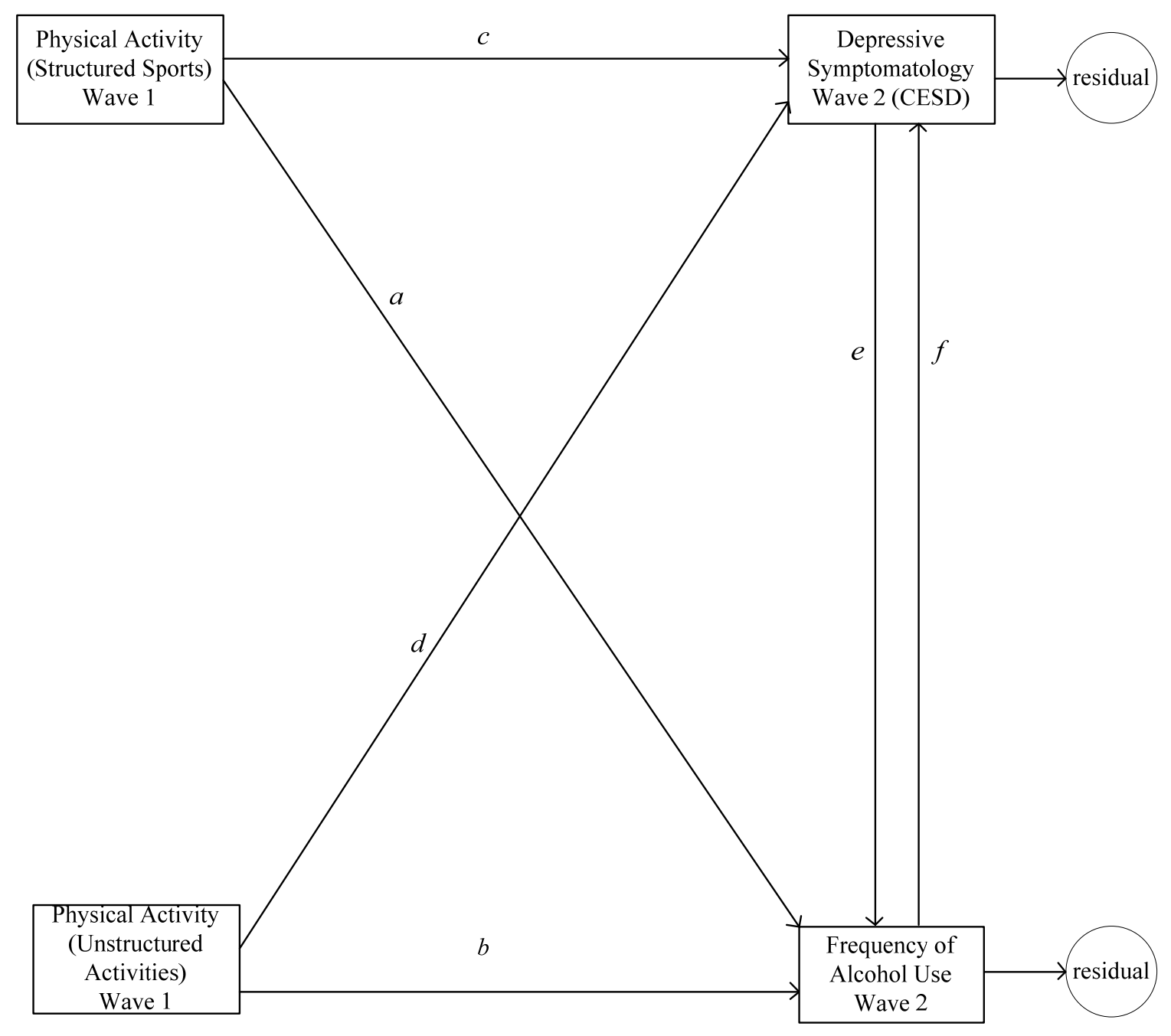


Figure 2. Conceptual Model with Binge Drinking as Outcome




Figure 3. Model with Frequency of Alcohol Use as Outcome for Males

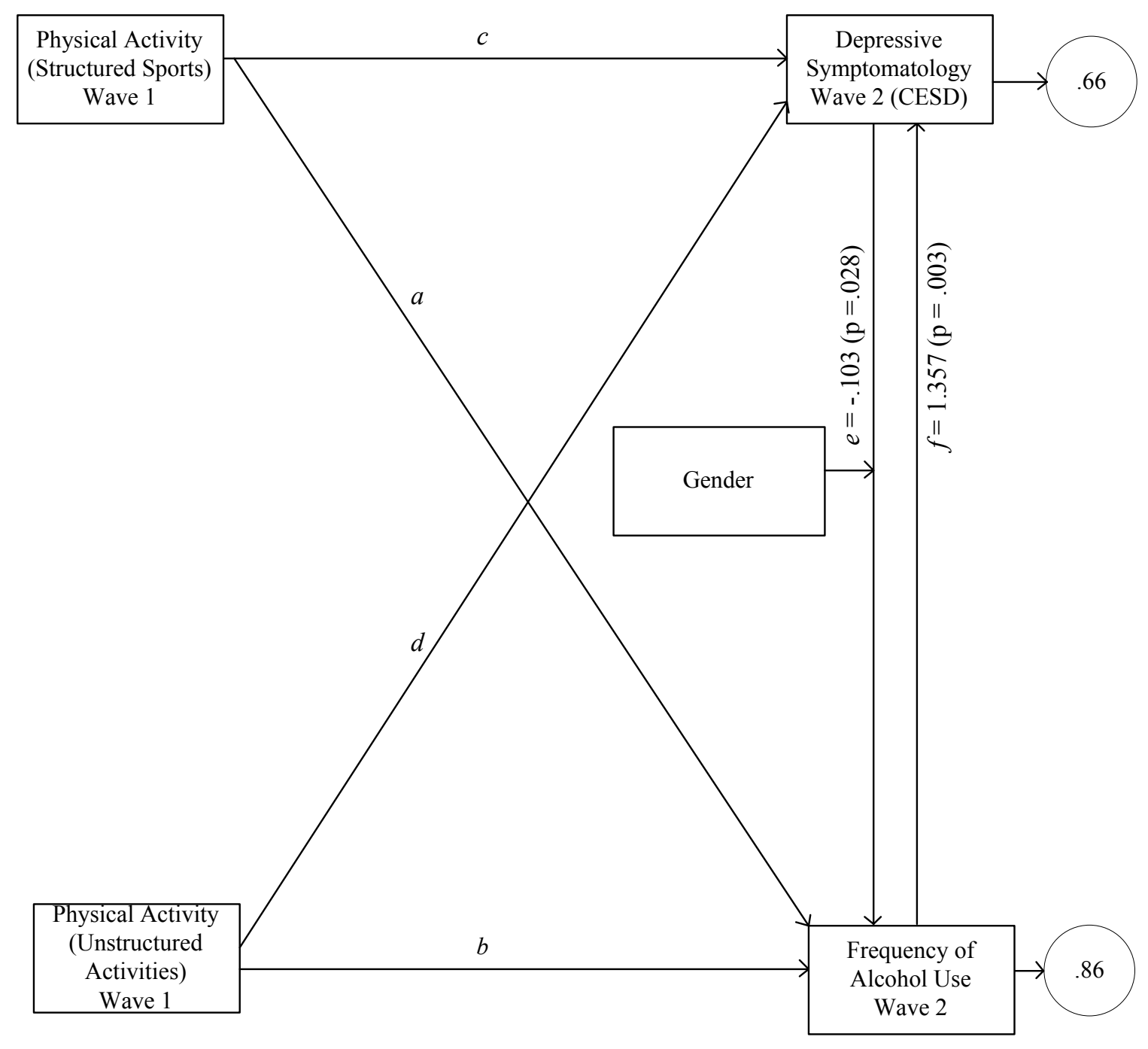


Figure 4. Model with Frequency of Alcohol Use as Outcome for Females

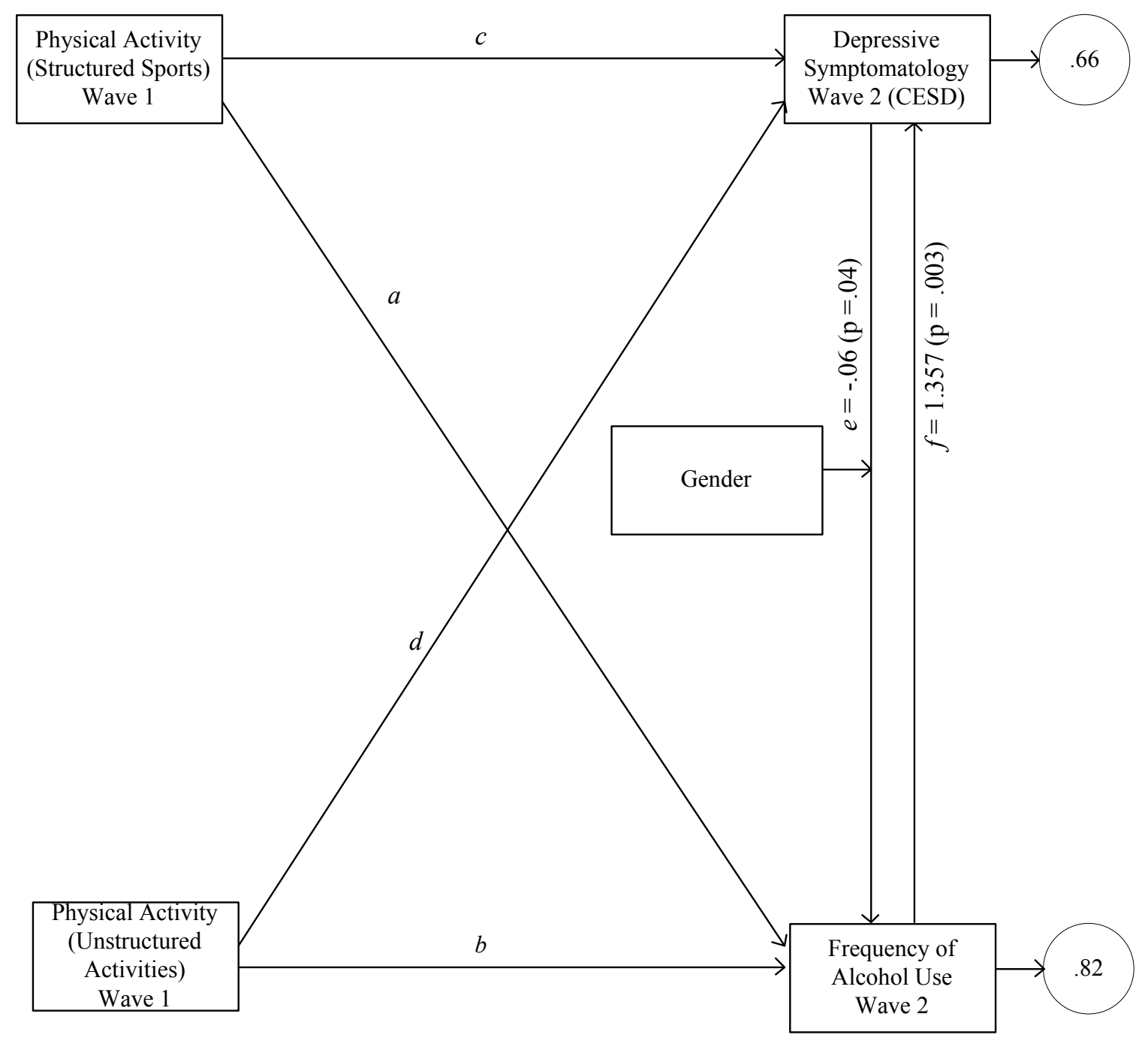


Figure 5. Model with Frequency of Binge Drinking as Outcome for Males

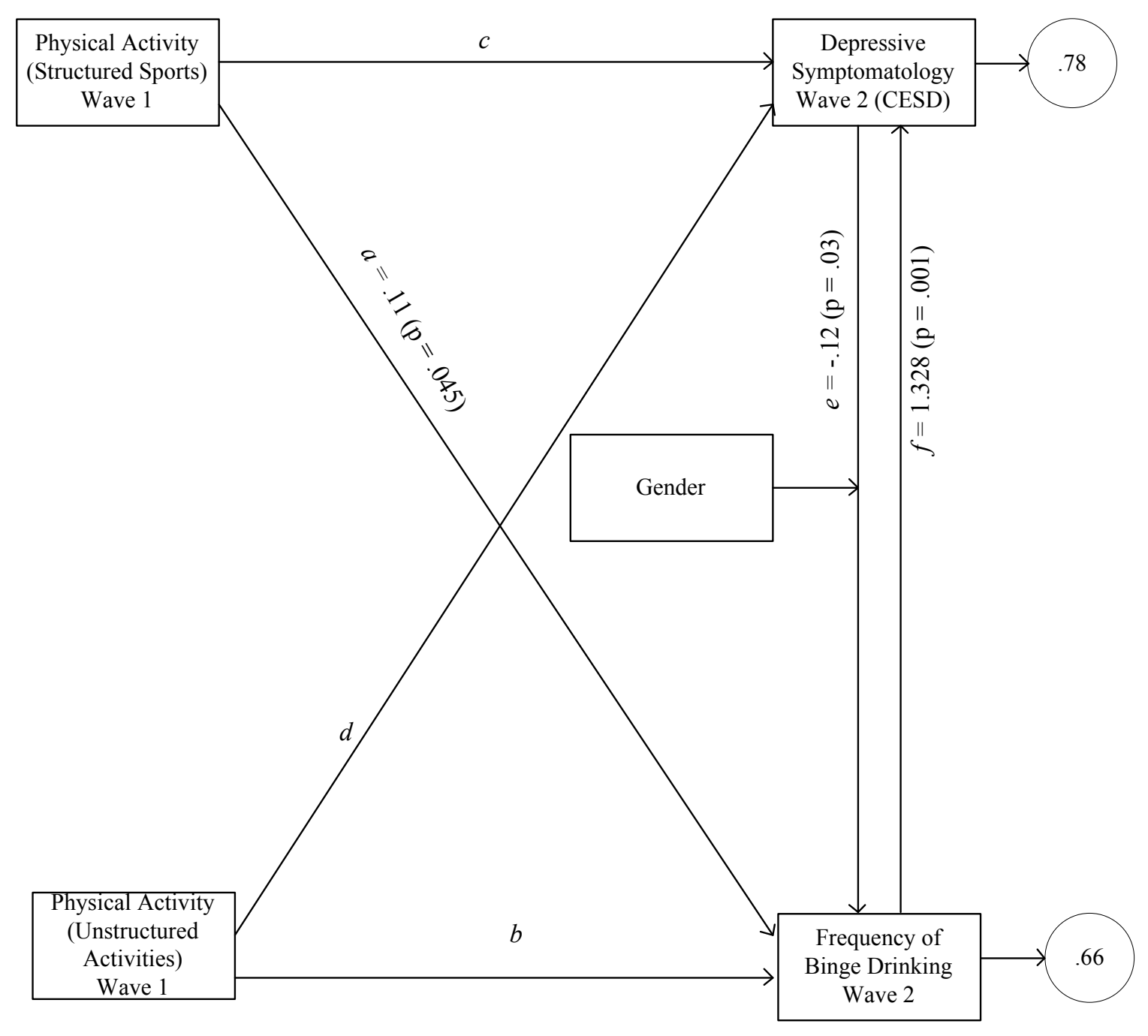


Figure 6. Model with Frequency of Binge Drinking as Outcome for Females

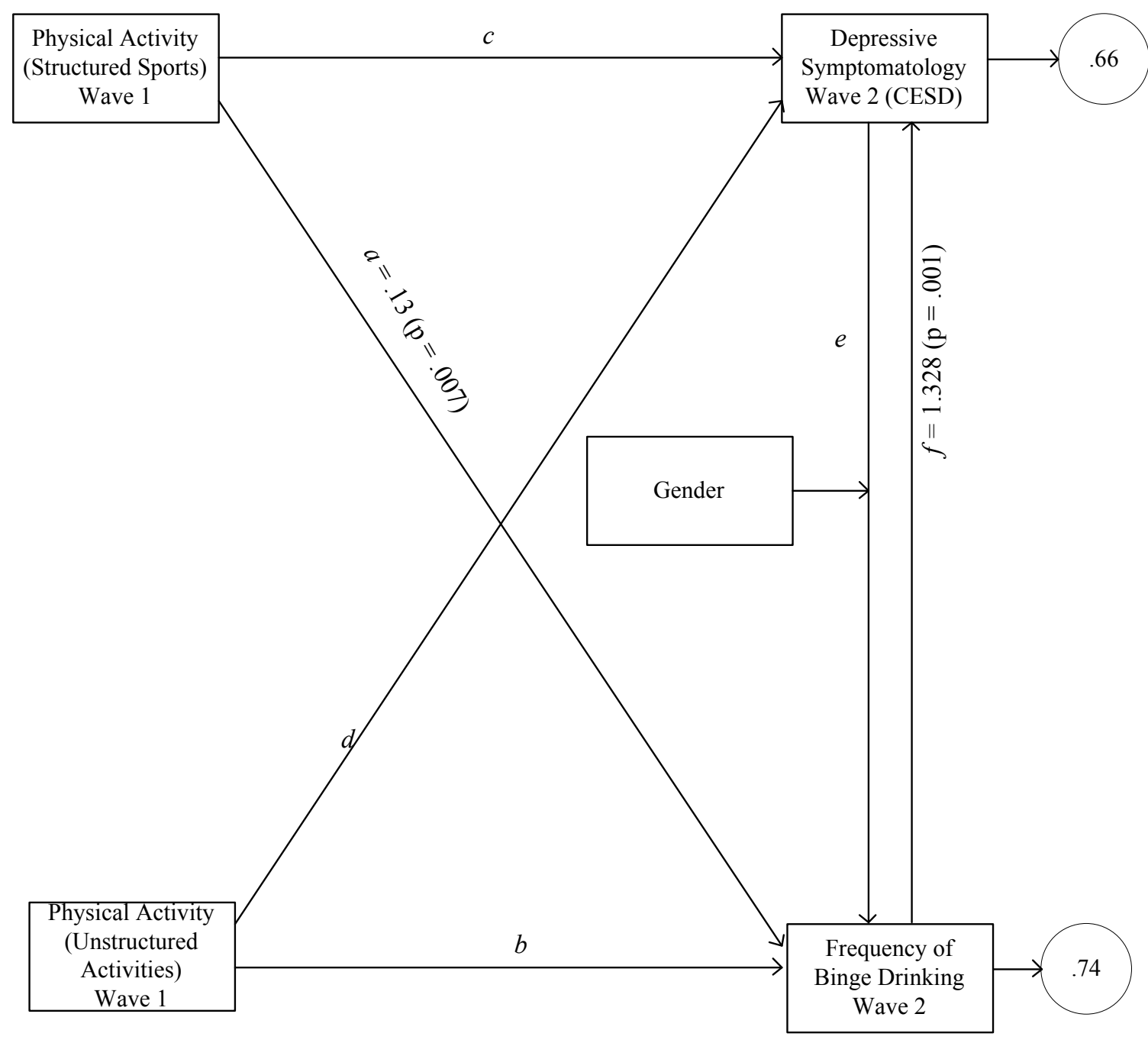




\section{REFERENCES}

Ainsworth, B.E., Haskell, W.L., Whitt, M.C., Irwin, M.L., Swartz, A.M., Strath, S.J., ... Leon, A.S. (2000). Compendium of physical activities: an update of activity codes and MET intensities. Medicine and Science in Sports and Exercise, 32(9), 498504. doi:10.1097/00005768-200009001-00009

Allison, K.R., Adlaf, E.M., Irving, H.M., Hatch, J.L., Smith, T.F., Dwyer, J.M., \& Goodman, J. (2005). Relationship of vigorous physical activity to psychological distress among adolescents. Journal of Adolescent Health, 37, 164-166. doi: 10.1016/j.jadohealth.2004.08.017.

Anderssen, N. (1993). Perceptions of physical education classes among young adolescents: do physical education classes provide equal opportunities to all students? Health Education Research, 8, 167-179.

Andersen, R. E., Crespo, C.J., Bartlett, S.J., Cheskin, L.J., \& Pratt, M. (1998). Relationship of physical activity and television watching with body weight and level of fatness among children: results from the Third National Health and Nutrition Examination Survey. Journal of the American Medical Association, 279 (12), 938-942. doi: 10.1001/jama.279.12.938

Angold, A., Costello, E.J., \& Erkanli, A. (1999). Comorbidity. Journal of Child Psychology \& Psychiatry, 40, 57-87. doi: 10.1111/1469-7610.00424

Arthur, M.W., Hawkins, J.D., Pollard, J.A., Catalno, R.F., \& Bagioni, A.J. (2002). Measuring risk and protective factors for substance use, delinquency, and other adolescent problem behaviors: The communities that care youth survey. Evaluation Review, 26 (6), 575-601. doi:10.1177/0193841X0202600601

Asparouhov, T. (2005). Sampling weights in latent variable modeling. Structural Equation Modeling, 12, 411-434. doi: org/10.1207/s15328007sem1203_4

Avenevoli, S., Knight, E., Kessler, R.C., \& Merikangas, K.R. (2008). Epidemiology of depression in children and adolescents. In J.R.Z. Abela \& B.L. Hankin (Eds.), Handbook of depression in children and adolescents (pp. 6-34). NY: The Guilford Press.

Barber, B.L., \& Eccles, J.S. (1999). Student council, volunteering, basketball, or marching band: What kind of extracurricular involvement matters? Journal of Adolescent Research, 14, 10-43.

Barber, B. L., Eccles, J. S., \& Stone, M. R. (2001). Whatever happened to the jock, the brain, and the princess? Young adult pathways linked to adolescent activity 
involvement and social identity. Journal of Adolescent Research, 16(5), 429-455. doi:10.1177/0743558401165002

Barber, B.L., Stone, M.R., Hunt, J.E., \& Eccles, J.S. (2005). Benefits of activity participation: The role of identity affirmation and peer group norm sharing. In J. Mahoney, R.W. Larson, \& J.S. Eccles (Eds.), Organized activities as contexts of development: Extracurricular activities, after-school and community programs (pp. 185-210). Hillsdale, NJ: Erlbaum.

Baron, R.M., \& Kenny, D.A. (1986). The moderator-mediator variable distinction in social psychological research: Conceptual, strategic and statistical considerations. Journal of Personality and Social Psychology, 51, 1173-1182. Retrieved from http://www.public.asu.edu/ davidpm/classes/psy536/Baron.pdf

Bartko, W.T., \& Eccles, J.S. (2003). Adolescent participation in structured and unstructured activities: A person oriented analysis. Journal of Youth and Adolescence, 32, 233-241.

Bearman, P.S., Jones, J., \& Udry, R.J. (1997). The National Longitudinal Study of Adolescent Health: Research Design. Carolina Population Center. University of North Carolina at Chapel Hill. Retrieved from http://www.cpc.unc.edu/projects/addhealth/design.html.

Benard, B.(2004). Resilience: A universal capacity. Bonnie, B. (Ed). Resiliency: What we have learned (pp. 7-12). WestEd.

Bhatia, S.K., \& Bhatia, S.C. (2007). Childhood and adolescent depression. American Family Physician, 75, 73-80.

Biehl, M.C., Natsuaki, M.N., \& Ge, X. (2007). The influence of pubertal timing on alcohol use and heavy drinking trajectories. Journal of Youth and Adolescence, 36, 153- 167. doi: 10.1007/s10964-006-9120-z

Bliss, D.L. (2007). Empirical research on spirituality and alcoholism: A review of the literature. Journal of Social Work Practice, 7, 5-24.

Bollen, K. \& Long, S. (1993). Testing structural equation models. Newbury Park: Sage.

Brook, D.W., Brook, J.S., Zhang, C., Cohen, P., \& Whiteman, M. (2002). Drug use and the risk of major depressive disorder, alcohol dependence, and substance use disorders. Archives of General Psychiatry, 59, 1039-1044.

Byrne, A., \& Byrne, D.G. (1993). The effect of exercise on depression, anxiety and other mood states: a review. Journal of Psychosomatic Research, 37(6), 565-574. doi: 10.1016/0022-3999(93)90050-P 
Caspersen, C.J., Powell, K.E., \& Christensen, G.M. (1985). Physical activity, exercise, and physical fitness: Definitions and distinctions for health-related research. Public Health Reports 100, 126-131. Retrieved from http://www.ncbi.nlm.nih.gov/pmc/articles/PMC1424733/pdf/pubhealthrep00100$\underline{0016 . p d f}$

Chantala, K., \& tabor, J., (1999). Strategies to perform a design-based analysis using the add health data. Carolina Population Center, University of North Carolina at Chapel Hill.

Chassin, L., Pitts, S., \& Prost, J. (2002). Binge drinking trajectories from adolescence to emerging adulthood in a high-risk sample: Predictors and substance abuse outcomes. Journal of Consulting and Clinical Psychology, 70, 67-78.

Chassin, L., Flora, D.B., \& King, K.M. (2004). Trajectories of alcohol and drug use and dependence from adolescence to adulthood: The effects of familial alcoholism and personality. Journal of Abnormal Psychology, 113, 483-498. doi:10.1037/0021-843X.113.4.483

Centers for Disease Control and Prevention. Participation in high school physical education-United States, 1991-2003. (2004). MMWR Morbidity Mortality Weekly Rep, 53, $844-847$.

Center for Disease Control and Prevention. (2008). Child Development. Retrieved September 28, 2009, from http://www.cdc.gov/ncbddd/child/earlyadolescence.htm

Center for Disease Control and Prevention (2011). National Center for Chronic Disease Preventions and Health Promotion, Division of Adolescent and School Health. Retrieved December 22, 2011, from http://www.cdc.gov/healthyyouth/adolescenthealth/index.htm

Chen, C.Y., Storr, C.L., Liu, C.Y., Chen, K.H., Chen, W.J., Lin, K.M. (2011). Differential relationships of family drinking with alcohol expectancy among urban school children. BMC Public Health, 11, 87-96. doi: 10.1186/1471-245811-87.

Clark, D.B., \& Bukstein, O.G. (1998). Psychopathology in adolescent alcohol abuse and dependence. Alcohol Research \& Health, 22, 117-121.

Collingwood, T.R., Sunderlin, J., Reynolds, R., Kohl, H.W. (2000). Physical Training as a Substance Abuse Prevention Intervention for Youth. Journal of Drug Education, 30, 435-451. doi:10.2190/RVUE-9XW7-TYRQ-EJR8 
Compas, B.E. (2004). Processes of risk and resilience during adolescence: linking individuals and contexts. In $2^{\text {nd }}$ ed. R.L. \& L.S (Eds.), In Handbook of Adolescent Psychology (pp.263-296). John Wiley. New York.

Costello, E.J., Erkanli, A., Federman, E., \& Angold, A. (1999). Transition into adolescent problem drinking: The role of psychosocial risk and protective factors. Journal of Studies on Alcohol, 60, 480-490.

Costello, E.J., Erkanli, A., \& Angold, A. (2006). Is there an epidemic of child or adolescent depression? Journal of Child Psychology and Psychiatry, 47, 12631271. doi: 10.1111/j.1469-7610.2006.01682.x

Craft, L.L., \& Landers, D.M. (1998). The effects of exercise on clinical depression and depression resulting from mental illness: a metanalysis. Medicine \& Science in Sports \& Exercise, 20, 339-357.

Craft, L. (2005). Exercise and clinical depression: examining two psychological mechanisms. Psychology of Sport and Exercise, 6, 151-171. doi: 10.1016/j.psychsport.2003.11.003

Cranford, J.A., Zucker, R.A., Jester, J.M., Puttler, L.I., \& Fitzgerald, H.E. (2010). Parental alcohol involvement and adolescent alcohol expectancies predict alcohol involvement in male adolescents. Psychology of Addictive Behaviors, 24, 386396. doi: 10.1037/a0019801

Crum R.M., Green K.M., Storr C.L., Chan Y.F., Ialongo N., Stuart E.A., \& Anthony J.C. (2008). Depressed mood in childhood and sub- sequent alcohol use through adolescence and young adulthood. Archives in General Psychiatry, 65, 702-712. doi: 10.1001/archpsyc.65.6.702

Dams-O'Connor, K., Martin, J.L., \& Martens, M.P. (2007). Social norms and alcohol consumption among intercollegiate athletes: The role of athlete and nonathlete reference groups. Addictive Behaviors, 32, 2657-2666.

Darling, N., Caldwell, L. L., \& Smith, R. (2005). Participation in school-based extracurricular activities and adolescent adjustment. Journal of Leisure Research, 37, 51-76. doi.org/10.1007/s10964-005-7266-8

Dauber, S., Houge, A., Paulson, J.F., \& Leiferman, J.A. (2009). Typologies of alcohol use in White and African American adolescent girls. Substance Use \& Misuse, 44, 1121-1141. doi: 10.1080/10826080802494727

Dawber, T. R., \& Kannel, W. B. (1966). The Framingham study: An epidemiological approach to coronary heart disease. Circulation, 34, 553-555. Retrieved from http://circ.ahajournals.org/cgi/reprint/34/4/553.pdf 
De Bellis, M.D., Narasimhan, A., Thatcher, D.L., Keshavan, M.S., Soloff, P., \& Clark, D.B. (2005). Prefrontal cortex, thalamus, and cerebellar volumes in adolescents and young adults with adolescent-onset alcohol use disorders and comorbid mental disorders. Alcoholism: Clinical and Experimental Research, 29, 15901600. doi: 10.1097/01.alc.0000179368.87886.76

Diego, M., Field, T., \& Sanders, C. (2003). Academic performance, popularity, and depression predict adolescent substance use. Adolescence, 38(149), 35-42.

Donovan, J.E. (2004). Adolescent alcohol initiation: A review of psychosocial risk factors. Journal of Adolescent Health, 35, 569-581.

Eccles, J.S., Barber, B.L., Stone, M., \& Hunt, J. (2003). Extracurricular activities and adolescent development. Journal of Social Issues, 59, 865-889.

Edwards, A.C., Sihvola, E., Korhonen, T., Pulkkinen, L., Moilanen, I., Kapiro, J., Rose, R.J., \& Dick, D.M. (2011). Depressive symptoms and alcohol use are genetically and environmentally correlated across adolescence. Behavioral Genetics, 41, 476487. doi: 10.1007/s10519-010-9400-y

Enders, C. (2010). Applied missing data analysis (methodology in the social sciences). New York NY: Guilford Press.

Essau, C.A., Conradt, J., \& Peterman, F. (2000). Frequency, comorbidity, and psychosocial impairment of depressive disorders in adolescents. Journal of Adolescent Research, 15, 470-481. doi:10.1016/S0887-6185(99)00039-0

Faden, V.B. (2006). Trends in Initiation of Alcohol Use in the United States 1975-2003. Alcoholism, Clinical and Experimental Research, 30, 1011-1022. doi:10.1111/j.1530-0277.2006.00115.x

Farmer, M.E., Locke, B.Z., Moscicki, E.K., Dannenberg, A.L., Larson, D.B., \& Radloff, L.S. (1988). Physical activity and depressive symptoms: The NHANES I Epidemiologica Follow-up Study. American Journal of Epidemiology, 128 (6), 1340-1351. Retrieved from http://aje.oxfordjournals.org/content/128/6/1340.full.pdf

Festinger, T. (1984). No one ever asked us: A postscript to the foster care system. New York: Columbia University Press.

Field, T., Diego, M., \& Sanders, C. (2001). Adolescent depression and risk factors. Adolescence, 36, 491-498.

Fincham, F.D. (1999). Stages of research. Presentation at the Society for Research on Child Development. 
Fox, K.R. (1999). The influence of physical activity on mental well-being. Public Health Nutrition, 2, 411-418. doi:10.1017/S1368980099000567

Franko, D.L., Dorer, D.J., Keel, P.K., Jackson, S., Manso, M.P., \& Herzog, D.B. (2005). How do eating disorders and alcohol use disorder influence each other? International Journal of Eating Disorders, 38(3), 200-207. doi: 10.1002/eat.20178

Fredricks, J. A., \& Eccles, J. S. (2006). Is extracurricular participation associated with beneficial outcomes? Concurrent and longitudinal relations. Developmental Psychology, 42, 698-713. doi:10.1037/0012-1649.42.4.698

Gauvin, L., \& Spence, J.C. (1996). Physical activity and psychological well-being: Knowledge base, current issues and caveats. Nutrition Reviews, 54 (4), 53-65. doi: 10.1111/j.1753-4887.1996.tb03899.x

Goldfield, G.S., Henderson, K., Buchholz, A., Obeid, N., Ngyuen, H., \& Flament, M.F. (2011). Physical activity and psychological adjustment in adolescents. Journal of Physical Activity \& Health, 8, 157-163.

Goldman, M.S., Darkes, J., \& Del Boca, F.K. (1999). Expectancy mediation of biopsychosocial risk for alcohol use and alcoholism. In I. Kirsch (Ed.), How expectancies shape experience (pp. 233-262). Washington, D.C.: American Psychological Association.

Goodwin, R.D. (2003). Association between physical activity and mental disorders among adults in the United States. Preventive Medicine, 36, 698-703. doi:10.1016/S0091-7435(03)00042-2

Gordon-Larsen, P., McMurray, R., and Popkin, B. (2000). Determinants of adolescent physical activity and inactivity patterns. Pediatrics, 105, 83-91. doi: 10.1542/peds.105.6.e 83

Gore, S., Farrell, F., \& Gordon, J. (2001). Sports involvement as protection against depressed mood. Journal of Research on Adolescence, 11, 119-130. doi:10.1111/1532-7795.00006

Guo, J., Collins, L.M., Hill, K.G., \& Hawkins, J.D. (2000). Developmental Pathways to Alcohol Abuse and Dependence in Young Adulthood. Journal of Studies on Alcohol and Drugs, 61, 799-808. Retrieved from http://www.ncbi.nlm.nih.gov/pmc/articles/PMC1975961/pdf/nihms18669.pdf

Hacker, K.A., Suglia, S.F., Fried, L.E., Rappaport, N., \& Cabral, H. (2006). Developmental differences in risk factors for suicide attempts between ninth and eleventh graders. Suicide and Life Threatening Behavior, 36, 154-166. doi: 10.1521/suli.2006.36.2.154 
Hair, J.F., Black, B., Babin, B., Anderson, R.E., \& Tatham, R.L. (2006), Multivariate Data Analysis, Sixth Edition, Pearson Prentice Hall: Upper Saddle River, NJ.

Hallfors, D. D., Waller, M. W., Bauer, D., Ford, C. A., \& Halpern, C. T.(2005). Which comes first in adolescence-Sex and Drugs or Depression? American Journal of Preventive Medicine, 29, 163-170. doi:10.1016/j.amepre.2005.06.002

Ham, L.S., \& Hope, D.A. (2003). College students and problematic drinking: A review of the literature. Clinical Psychology Review, 23, 719-759.

Harris, K.M., Halpern, C.T., Whitsel, E., Hussey,J., Tabor, J., Entzel, P., \& Udry, J.R. (2009). The National Longitudinal Study of Adolescent Health: Research Design. Retrieved from: http://www.cpc.unc.edu/projects/addhealth/design

Harter, S., \& Monsour, A. (1992). Developmental analysis of conflict caused by opposing self-attributes in the adolescent self-portrait. Developmental Psychology, 28, 251260. doi: apa.org/psycinfo/1992-23056-001

Haslam, D.W., \& James, P.T. (2005). Obesity. Lancet, 366, 1197-1209. doi:10.1016/S0140-6736(05)67483-1

Haugland, S., Wold, B., \& Torsheim, T. (2003). Relieving the Pressure? The role of physical activity in the relationship between school-related stress and adolescent health complaints. Research Quarterly for Exercise and Sport, 74, 127-135.

Hawkins, J.D., Catalano, R.F., and Miller, J.Y. (1992). Risk and protective factors for alcohol and other drug problems in adolescence and early adulthood: Implications for substance abuse prevention. Psychological Bulletin, 112, 64-105. doi:10.1037/0033-2909.112.1.64

Hingson, R., \& Kenkel, D. (2004). Social health and economic consequences of underage drinking. In: National Research Council and Institute of Medicine, Bonnie, R.J., and O'Connell, M.E., eds. Reducing Underage Drinking: A Collective Responsibility. Washington, DC: National Academies Press, 2004, 351-382.

Honaker, J., Joseph, A., King, G., Scheve, K., Singh, N., \& Amelia, A. (2003). A program for missing data. Department of Government, Harvard University.

Jenson, J.M., \& Fraser, M.W. (2006). A risk and resilience framework for child, youth, and family policy. In J.M. Jenson, \& M.W. Fraser (Eds.). Social Policy for children and families (pp. 1-18). Sage Publishers. 
Jessor, R. (1992). Risk behavior in adolescence: A psychosocial framework for understanding and action. Developmental Review, 12, 374-390. doi:10.1016/1054$139 \mathrm{X}(91) 90007-\mathrm{K}$

Johnston LD, O'Malley PM, Bachman JG, and Schulenberg JE. 2007. Data tables from the 2007 Monitoring the Future Survey. Ann Arbor, MI: University of Michigan News and Information Services. [On-line]. Available: www.monitoringthefuture.org; accessed 07/21/09.

Johnston, L. D., O'Malley, P. M., Bachman, J. G., \& Schulenberg, J. E. (2008). Monitoring the future national results on adolescent drug use: Overview of key findings, 2007, 1-70. (NIH Publication No. 08-6418). ). Bethesda, MD: National Institute on Drug Abuse, U.S. Department of Health and Human Services.

Johnston, L.D., O’Malley, P.M., Bachman, J.G., \& Schulenberg, J.E. (2009). Monitoring the Future National Results on Adolescent Drug Use: Overview of Key Findings, 2008, 1-73. (NIH Publication No. 09-7401). Bethesda, MD: National Institute on Drug Abuse, U.S. Department of Health and Human Services.

Jones, B.T., Corbin, W., \& Fromme, K. (2001). A review of expectancy theory and alcohol consumption. Addictions, 96, 57-72.

Joreskog, K. \& Sorbom, D, (1996). User's manual for LISREL. Chicago: Scientific Software.

Keenan-Miler, D., Hammen, C., \& Brennan, P. (2007). Health outcomes related to early adolescent depression. Journal of Adolescent Health, 42, 256-262. doi: 10.1016/j.jadohealth.2007.03.015

Kessler, R.C., \& Walter, E.E. (1998). Epidemiology of DSM III-R major depression and minor depression among adolescents and young adults in the national comorbidity survey. Depression and Anxiety, 7, 3-14. doi:10.1002/(SICI)15206394(1998)7:1<3::AID-DA2>3.0.CO;2-F

Kessler, R.C., Avenevoli, S., \& Merikangas, K.R. (2001). Mood disorders in children and adolescents: An epidemiologic perspective. Biological Psychiatry, 49, 1002-1014. doi:10.1016/S0006-3223(01)01129-5

Kessler, R.C., Berglund, P., Demier, O., Jin,R., Merikangas, K.R., \& Walters, E.E. (2005). Lifetime prevalence and age-of-onset distributions of DSM-IV disorders in the National Comorbidity Survey replication. Archives of General Psychiatry, 62, 593- 602 .

Khantzian, E. (1997). The self-medication hypothesis of substance use disorders: A reconsideration and recent applications. Harvard Psychiatry, 4, 231-244. 
Khantzian, E. (2003). The self-medication hypothesis revisited: The daily diagnosed patient. Primary Psychiatry, 10, 47-54.

King, G., Honaker, J., Joseph, A. \& Scheve, K. (2001). Analyzing incomplete political science data: An alternative algorithm for multiple imputation. American Political Science Review, 95, 49-69. doi: 10.2307/3117628

Kirkcaldy, B.D., Shepard, R.J., \& Siefen, R.G. (2002). The relationship between physical activity and self-image and problem behavior among adolescents. Social Psychiatry and Psychiatric Epidemiology, 37, 544-550. doi:10.1007/s00127-0020554-7

Kleiber, D.A., Larson, R.W., \& Csikszentmihalyi, M. (1986). The experience of leisure in adolescence. Journal of Leisure Research, 18, 169-176.

Kleiber, D.A., Caldwell, L.C., \& Shaw, S.M. (1993). Leisure meanings in adolescence. Society and Leisure, 18, 169-176.

Kleiber, D.A. (1999). Leisure experience and human development: A dialectical interpretation. New York: Basic Books.

Knopf, D., Park, M.J., \& Paul Mulye, T. A. (2008). Mental Health Profile of Adolescents: 2008 Brief. National Adolescent Health Information Center. Retrieved from http://nahic.ucsf.edu/downloads/MentalHealthBrief.pdf.

Knutson, K. (2005). Sex differences in the association between sleep and body mass index in adolescents. The Journal of Pediatrics, 147, 830-834. doi: 10.1016/j.jpeds.2005.07.019

Korhanen, T., Kujala, U.M., Rose, R.J., \& Jaakko, K. (2009). Physical activity in adolescence as a predictor of alcohol and illicit drug use in early adulthood: A longitudinal population based twin study. Twins Research Human Genetics, 12, 261-268.

Kraemer, H.C., Stice, E., Kazdin, A.E., Offord, D.R., \& Kupfer, D.J. (2001). How do risk factors work together? Mediators, moderators, independent, overlapping, and proxy-risk factors. American Journal of Psychiatry, 158, 848-856. Retrieved from http://ajp.psychiatryonline.org/cgi/reprint/158/6/848

Kraemer, H.C., Wilson, G.T., Fairburn, C.G., \& Agras, W.S. (2002). Mediators andmoderators of treatment effects in randomized clinical trials. Archives of General Psychiatry, 59, 877-883. Retrieved from http://www.personal.kent.edu/ dfresco/CRM_Readings/Archives_Kraemer 2002. pdf 
Kumpulainen, K. (2000). Psychiatric symptoms and deviance in early adolescence predict heavy alcohol use 3 years later. Addiction, 95, 1847-1857.

doi:10.1046/j.1360-0443.2000.9512184713.x

Lewis, T.F. (2008). An explanatory model of student-athlete drinking: The role of team leadership, social norms, perception of risk, and coaches' attitudes toward alcohol consumption. College Student Journal, 42, 818-831.

Lewinsohn, P. M., Hops, H., Roberts, R. E., Seeley, J. R., \& Andrews, J. A. (1993). Adolescent psychopathology: I. Prevalence and incidence of depression and other DSM-III-R disorders in high school students. Journal of Abnormal Psychology, 102, 133-144. doi:10.1037/0021-843X.102.1.133

Luthar, S.S., \& Cicchetti, D.(2000). The construct of resilience: Implications for interventions and social policies. Developmental Psychopathology, 12, 857-885. Retrieved from http://www.ncbi.nlm.nih.gov/pmc/articles/PMC1885202/pdf/nihms-21559.pdf

Luthar S, Cicchetti D, Becker B. (2000). The construct of resilience: A critical evaluation and guidelines for future work. Child Development, 71, 543-562. doi:10.1111/1467-8624.00164

MacKinnon, D. P., Lockwood, C. M., Hoffman, J. M., West, S. G., \& Sheets, V. (2002). A comparison of methods to test mediation and other intervening variable effects. Psychological Methods, 7, 83-104. doi: 10.1037/1082-989X.7.1.83

MacMahon, J.R., \& Gross, R.T. (1988). Physical and psychological effects of aerobic exercise in delinquent adolescent males. American Journal of Diseases in Children, 142, 1351-1366.

Marten, M.P., Dams-O’Connor, K., \& Beck, N.C. (2006). A systematic review of college student-athlete drinking: Prevalence rates sport-related factors, and interventions. Journal of Substance Abuse Treatment, 21, 305-316.

Masten, A. S., Best, K. M., \& Garmezy, N. (1990). Resilience and development: Contributions from the study of children who overcome adversity. Development and Psychopathology, 2, 425-444. doi:10.1017/S0954579400005812

Mays, D. \& Thompson, N.J. (2009). Alcohol-related risk behaviors and sport participation among adolescents: An analysis of 2005 Youth Risk Behavior Survey Data. Journal of Adolescent Health, 44, 87-89. doi: 10.1016/j.jadohealth.2008.06.011

Mays, D., Thompson, N., Kushner, H.I., Mays, D.F., Farmer, D., \& Windle, M. (2010). Sports specific factors, perceived peer drinking, and alcohol-related behaviors 
among adolescents participating in school-based sports in southwest Georgia. Addictive Behaviors, 35, 235-241.

McDermott, R.J., Hawkins, W.E., Marty, P.J., Littlefield, E.A., Murray, S., \& Williams, T.K. (1990). Health Behavior correlates of depression in a sample of high school students. Journal of School Health, 60, 414-417. doi:10.1111/j.17461561.1990.tb05961.x

McDonald, D.G., \& Hodgdon, J.A. (1991). Psychological effects of aerobic fitness training: Research and theory. New York: Springer-Verlag.

Moore, M. J., \& Werch, C. E. (2005). Sport and physical activity participation and substance use among adolescents. Journal of Adolescent Health, 35, 486-493. doi:10.1016/j.jadohealth.2004.02.031

Motl, R.W., Birnbaum, A.S., Kubik, M.Y., \& Dishman, R.K. (2004). Naturally occurring changes in physical activity are inversely related to depressive symptoms during early adolescence. Psychosomatic Medicine, 66, 336-342. doi:10.1097/01.psy.0000126205.35683.0a

Mulye, T.P., Park, M.J., Nelson, C.D., Adams, S.H., Irwin, C.E., \& Brindis, C.D. (2009). Trends in Adolescent and Young Adult Health in the United States. Journal of Adolescent Health, 45, 8-24. doi:10.1016/j.jadohealth.2009.03.013

Murray, N.G., Kelder, S.H., \& Meyers, M.C. (1998). Type of physical activity and level of depression in suburban $9^{\text {th }}$ and $10^{\text {th }}$ grade students. Medicine $\&$ Science in Sports \& Exercise, 30, S117.

National Association of Social Work (2009). National standards for social work practice with clients with substance use disorders. Retrieved from http://www.socialworkers.org/practice/standards/NASWATODStatndards.pdf

National Household Survey on Drug Abuse. (2002, April 11). Binge drinking among underage persons. Retrieved from http://oas.samhsa.gov/2k2/AlcBinge/AlcBinge.htm

National Institute on Alcohol Abuse and Alcoholism. (2005). Alcohol and development in youth-a multidisciplinary overview. Alcohol Research \& Health, 28, 2004/2005. Retrieved from http://pubs.niaaa.nih.gov/publications/arh283/111$\underline{120 . h t m}$

National Center for Health Statistics. (2005). Health, United States, 2005: With chartbook on trends in the health of Americans (DHHS Publication No. 20051232). Washington, DC: U.S. Government Printing Office. 
Nelson, T.F., \& Wechsler, H. (2001). Alcohol and college athletes. Medicine and Science in Sports and Exercise, 33, 43-47.

Nelson, M.C., \& Gordon-Larsen, P. (2006). Physical Activity and Sedentary Behavior Patterns Are Associated With Selected Adolescent Health Risk Behaviors. Pediatrics, 117, 1281-1290. doi:10.1542/peds.2005-1692

Nelson, M.C., Gordon-Larsen, P., Adair, L., \& Popkin, B. (2005). Adolescent physical activity and sedentary behavior patterning and long-term maintenance. American Journal of Preventive Medicine, 28, 259-266. doi: 10.1016/j.amepre.2004.12.006

North, T.C., McCukkagh, P., \& Tran, Z.V. (1990). Effect of exercise on depression. Exercise and Sport Sciences Review, 18, 379-415.

Ozer, E.M., Zahnd, E.G., Adams, S.H., Husting, S.R., Wibbelsman, C.J., Norman, K.P., Smiga, S.M. .(2009). Are Adolescents Being Screened for Emotional Distress in Primary Care? Journal of Adolescent Health, 44, 520-527. doi: 10.1016/j.jadohealth.2008.12.016

Ozer, E.M., \& Irwin, C.E. (2009). Adolescent and young health: from basic health status to clinical interventions. In: Lerner, R.M., Steinberg, L., eds. Handbook of Adolescent Psychology (pp. 618-641). $3^{\text {rd }}$ ed. Hoboken, NJ: John Wiley \& Sons.

Paluska, S., \& Schwenk, T. (2000). Physical activity and mental health. Sports Medicine, 29, 167-180.

Pate, R.R., Davis, M.G., Robinson, T.N., Stone, E.J., McKenzie, T.L., \& Young, J.C. (2006). Promoting Physical Activity in Children and Youth A Leadership Role for Schools: A scientific statement from the American Heart Association Council on Nutrition, Physical Activity, and Metabolism (Physical Activity Committee) in collaboration with the Councils on Cardiovascular Disease in the Young and Cardiovascular Nursing. Circulation, 114, 1214-1224.

doi:10.1161/CIRCULATIONAHA.106.177052

Peretti-Watel, P., Beck, F., and Legleye, S. (2002). Beyond the U-curve: the relationship between sport and alcohol, cigarette and cannabis use in adolescents. Addiction, 97, 707-716. doi:10.1046/j.1360-0443.2002.00116.x

Phillips, W.T., Kiernan, M., \& King, A.C. (2003). Physical activity as a nonpharmacological treatment for depression: A review. Complementary Health Practice Review, 8, 1-14. doi: 10.1177/1076167502250792

Radloff, L.S. (1977). The CES-D scale: A self-report depression scale for research in the general population. Applied Psychological Measurement, 1, 385-401. doi: $10.1177 / 014662167700100306$ 
Raghunathan, T.E. (2004). What do we do with missing data? Some options for analysis of incomplete data. Annual Review of Public Health, 25, 99-117. doi:

10.1146/annurev.pubhealth.25.102802.124410

Rao, U., Daley, S.E., \& Hammen, C. (2000). Relationship between depression and substance use disorders in adolescent women during the transition to adulthood. Journal of the American Academy of Child Adolescent Psychiatry, 39, 215-222.

Resnick, MD., Bearman, P.S., Blum, R.W., Bauman, K.E., Harris, K., Jones, J., ... Udry, J.R. (1997). Protecting adolescents from harm: Findings from the National Longitudinal Study on Adolescent Health. Journal of the American Medical Association, 10, 823-832. doi:10.1001/jama.1997.03550100049038

Roberts, R.E. (1995). Depressive symptoms and suicidal ideation among Mexican-origin and Anglo adolescents. Journal of American Academy of Child and Adolescent Psychiatry, 34, 81-90. doi: 10.1097/00004583-199501000-00018

Rohde, P., Lewinsohn, P.M., \& Seeley, J.R. (1991). Comorbidity of unipolar depression: II. Comorbidity with other mental disorders in adolescents and adults. Journal of Abnormal Psychology, 100, 214-222.

Rohde, P., Lewinsohn, P.M., \& Seeley, J.R. (1996). Psychiatric comorbidity with problematic alcohol use in high school students. Journal of the American Academy of Child Adolescent Psychiatry, 35, 101-109.

Rutter, M. (1987). Psychosocial resilience and protective mechanisms. American Journal of Orthopsychiatry, 57, 316-331. doi:10.111/j.1939-0025.1987.tb03541.x

Sabo, D., Melnick, M.J., Miler, K.E., Farrell, M.P., and Barnes, G.M. (2002). Athletic participation and the health risks of adolescent males: A national study. International Journal of Men's Health, 1, 173-186.

Sallis, J.F., Prochaska, J.J., \& Taylor, W.C. (2000). A review of correlates of physical activity of children and adolescents. Medicine and Science in Sports and Exercise, 32, 963-975.

Sallis, J. F., M. J., Buono, M.J., Roby, J.J., Micale, F.G., Nelson, J.A. (1993). Seven-day recall and other physical activity self-reports in children and adolescents. Medicine \& Science in Sports \& Exercise, 25, 99-108.

Salmon, P. (2001). Effects of physical exercise on anxiety, depression, and sensitivity to stress: A unifying theory. Clinical Exercise Review, 21, 33-61. doi:10.1016/S0272-7358(99)00032-X 
Sanders, C.E., Field, T.M., Diego, M., \& Kaplan, M. (2000). The relationship of internet use to depression and social isolation among adolescents. Adolescence, 35, 237242.

Santos, M., Richards, C.S., \& Bleckley, M.K. (2007). Comorbidity between depression and disordered eating in adolescents. Eating Behaviors, 8, 440-449. doi:10.1016/j.eatbeh.2007.03.005

Seeley, J.R., Stice, E., \& Rohde, P. (2009). Screening for depression prevention: Identifying adolescent girls at high risk for future depression. Journal of Abnormal Psychology, 118, 161-170. doi: 10.1037/a0014741

Segal, E.A., Gerdes, K.E., \& Steiner, S. (2009). An introduction to the profession of social work: Becoming a change agent. In EL. Stromwall (Ed.) Mental Health Services (pp. 267-295). Belmont, CA: Brooks/Cole Cengage Learning.

Sieving, R.E., Beuhring, T., Resnick, M.D., Bearinger, L.H., Shew, M., Ireland, M., \& Blum, R.W. (2001). Development of adolescent self -report measures from the national longitudinal study of adolescent health. Journal of Adolescent Health, 28, 73-81. doi: 10.1016/S1054-139x(00)00155-5

Sihvola, E., Rose, R.J., Dick, D.M., Pulkkinen, L., Marttunen, M., Kaprio, J. (2008). Early-onset depressive disorders predict the use of addictive substances in adolescence: a prospective study of adolescent Finnish twins. Addiction ,103, 2045-2053. doi: 10.1111/j.1360-0443.2008.02363.x

Singh, A.K., Mahashwari, A., Sharma, N. \& Anand, K. (2006). Lifestyle associated risk factors in adolescents. Indian Journal of Pediatrics, 73, 901-906. doi:10.1007/BF02859283

Southwick, S.M., Vythilingam, M., \& Charney, D.S. (2005). The psychobiology of depression and resilience to stress: Implication for prevention and treatment. Annual Review of Clinical Psychology, 1, 255-291. doi:10.1146/annurev.clinpsy.1.102803.143948

Stathopoulou, G., Powers, M.B., Berry, A.C., \& Smits, J.A.J., \& Otto, M.W. (2006). Exercise interventions for mental health: A qualitative review. Clinical Psychology: Science and Practice, 13, 179-193. doi:10.111/j.14682850.2006.0021.x

Steptoe, A., \& Butler, N. (1996). Sports participation and emotional well-being in adolescents. Lancet, 347, 1789-1792. doi:10.1016/S0140-6736(96)91616-5

Straussner, S.L.A. (2001). The role of social workers in the treatment of addictions: A brief history. Journal of Social Work Practice in the Addictions, 1, 3-9. 
Substance Abuse and Mental Health Services Administration (1999). NHSDA Series: H9. Results from the 1998 National Household Survey on Drug Abuse. Office of Applied Studies, August 1999.

Substance Abuse and Mental Health Services Adminsitration. (2004). Results from the 2003 National Survey on Drug Use and Health: National Findings (Office of Applied Studies, NSDUH Series H-25, DHHS Publication No. SMA 04-3964). Rockville, MD.

Substance Abuse and Mental Health Services Administration, Office of Applied Studies (2008). Results from the 2007 National Survey on Drug Use and Health: National Findings (NSDUH Series H-34, DHHS Publication No. SMA 08-4343). Rockville, MD.

Substance Abuse and Mental Health Services Administration, Office of Applied Studies. (April 29, 2010). The OAS Report: A Day in the Life of American Adolescents: Substance Use Facts Update. Rockville, MD.

Substance Abuse and Mental Health Services Administration, Center for Behavioral Health Statistics and Quality. (April 28, 2011). The NSDUH Report: Major Depressive Episode and Treatment among Adolescents: 2009. Rockville, MD.

Sunstein, C.R. (2008). Adolescent risk-taking and social meaning: A commentary. Developmental Review, 28, 145-152.

Terzian, M.A., Andrews, K.M., \& Moore, K.A. (2011). Preventing multiple risky behaviors: An updated framework for policy and practice. Washington, DC: Child Trends.

Thomson, L.M., Pangrazi, R.P., Friedman, G., \& Hutchinson, N. (2003). Childhood depressive symptoms, physical activity and health related fitness. Journal of Sport \& Exercise Psychology, 25, 419-439.

Thompson, P.D., Buchner, D., Piña, I.L., Balady, G.J., Williams, M.A., Marcus, B.H., ... Wenger, N.K. (2003). Exercise and physical activity in the prevention and treatment of atherosclerotic cardiovascular disease: A statement from the council on clinical cardiology and the council on nutrition, physical activity, and metabolism. Circulation, 107, 3109-3116. doi:10.1161/01.CIR.0000075572.40158.77

Trost, S.G. (2003). Discussion paper for the development of recommendations for children's and youths' participation in health promoting physical activity. Canberra: Common- wealth Department of Health and Ageing. 
U.S. Department of Health and Human Services. (1996). Physical activity and health: a report of the Surgeon general, Atlanta: U.S. Department of Health and Human Services, CDC.

U.S. Department of Health and Human Services. (2000). Healthy People 2010. $2^{\text {nd }}$ ed. Understanding and improving health and objectives for improving health, Washington, DC: U.S. Department of Health and Human Services.

U.S. Department of Health and Human Services (2010). Healthy people 2020: Adolescent Health. Retrieved from http://healthypeople.gov/2020/topicsobjectives2020/overview.aspx?topicid=2

U.S. Department of Health and Human Services. The Surgeon General's Call to Action to Prevent and Reduce Underage Drinking. U.S. Department of Health and Human Services, Office of the Surgeon General, 2007.

U.S. Department of Health and Human Services. (2009). Alcohol Alert. A developmental perspective on underage alcohol use. Rockville, MD: U.S. Department of Health and Human Services. Full text available at www.niaaa.nih.gov

Vilhjalmsson, R., \& Horlindsson, T. (1998). Factors related to physical activity: a study of adolescents. Social Science \& Medicine, 4, 665-675. doi:10.1016/S02779536(98)00143-9

Wagner, E.F. (2008). Developmentally informed research on the effectiveness of clinical trials: A primer for assessing how developmental issues may influence treatment responses among adolescents with alcohol use problems. Pediatrics, 121, 337347. doi:10.1542/peds.2007-2243F

Werch, C., Moore, M., DiClemente, C.C., Owen, D.M., Jobli,E., \& Bledsoe, R. (2003). A Sport-Based Intervention for Preventing Alcohol Use and Promoting Physical Activity Among Adolescents. Journal of School Health, 73, 380-388. doi:10.1111/j.1746-1561.2003.tb04181.x

Werch, C., Moore, M.J., DiClemente, C.C., Bledsoe, R., \& Jobli, E.(2005). A Multihealth Behavior Intervention Integrating Physical Activity and Substance Use Prevention for Adolescents. Prevention Science, 6, 213-226. doi:10.1007/s11121-005-0012-3

Werner, E. E., \& Smith, R. S. (1992). Overcoming the odds: High risk children from birth to adulthood. Ithaca, NY: Cornell University Press.

Werner, E. E. (1994). Overcoming the odds. Journal of Developmental \& Behavioral Pediatrics, 15, 131-136. 
Werner E.E., \& Smith R.S. (2001). Journeys From Childhood to Midlife: Risk, Resilience and Recovery. Ithaca, NY: Cornell University Press.

Weyerer, S. (1992). Physical inactivity and depression in the community. Evidence from the upper Bavarian field study. International Journal of Sports Medicine, 13, 492496. doi: 10.1055/s-2007-1021304

Whitt-Glover,M.C., Taylor, W.C., Flyod, M.F., Yore, M.M., Yancey, A.K., \& Matthews, C.E. (2009). Disparities physical activity and sedentary behaviors among US children and adolescents: prevalence, correlates, and intervention implications. Journal of Public Health Policy, 30, 309-334.

Wight, R.G., Sepulveda, J.E., \& Aneshensel, C.S. (2004). Depressive symptoms: how do adolescents compare with adults? Journal of Adolescent Health, 34, 314-323. doi: 10.1016/j.jadohealth.2003.05.003

Wilkes, G. (2002). Abused child to non-abusive parent: Resilience and conceptual change. Journal of Clinical Psychology, 58, 261-278. doi:10.1002/jclp.10028

Windle, M., Spear, L.P., Fuligini, A., Brown, J.D., Pine, D.,...Dahl, R.E. (2008). Transitions into underage and problem drinking: Developmental processes and mechanism between 10 and 15 years of age. Pediatrics, 121, 273-289.

Yanovitzky, I., Stewart, L.P., \& Lederman, L.C. (2006). Social distance, perceived drinking by peers, and alcohol use by college students. Health Communication, 19, 1-10.

Zamboanga, B.L., \& Ham, L.S. (2008). Alcohol expectancies and context-specific drinking behaviors among female college athletes. Behavior Therapy, 39, 162170 .

Zucker, R.A., Kincaid, S.B., Fitzgerald, H.E., \& Bingham, C.R. (1995). Alcohol schema acquisition in preschoolers: Differences between children of alcoholics and children of nonalcoholics. Alcoholism: Clinical and Experimental Research, 19, 1011-1017. doi: 10.1111/j.1530-0277.1995.tb00982.x

Zucker, R.A., Donovan, J.E., Masten, A.S., Mattson, M.E., \& Moss, H.E. (2008). Early developmental processes and the continuity of risk for underage drinking and problem drinking. Pediatrics, 121, S252-S272. doi: 10.1542/peds.2007-2243B 


\section{APPENDICES}

\section{APPENDIX A: Physical Activity Measure}

1. During the past week, how many times did you go roller-blading, roller-skating, skate-boarding, or bicycling?

0 not at all

11 or 2 times

23 or 4 times

35 or more times

$6 \quad$ Refused

8 Don't know

2. During the past week, how many times did you play an active sport, such as baseball, softball, basketball, soccer, swimming, or football?

0 not at all

11 or 2 times

23 or 4 times

35 or more times

6 Refused

8 Don't know

3. During the past week, how many times did you do exercise, such as jogging, walking, karate, jumping rope, gymnastics or dancing?

0 not at all

11 or 2 times

23 or 4 times

35 or more times

6 Refused

8 Don't know 


\section{APPENDIX B: CES-D Measure}

1. You were bothered by things that usually don't bother you.

4 never

5 rarely

6 a lot of the time

7 most of the time or all of the time

$7 \quad$ Refused

9 Don't know

2. You didn't feel like eating, your appetite was poor.

4 never

5 rarely

6 a lot of the time

7 most of the time or all of the time

$7 \quad$ Refused

9 Don't know

3. You felt that you could not shake off the blues, even with help from your family and your friends.

0 never

1 rarely

2 a lot of the time

3 most of the time or all of the time

6 Refused

8 Don't know

4. You felt that you were just as good as other people.

$\begin{array}{ll}0 & \text { never } \\ 1 & \text { rarely } \\ 2 & \text { a lot of the time } \\ 3 & \text { most of the time or all of the time } \\ 6 & \text { Refused } \\ 8 & \text { Don't know }\end{array}$


5. You had trouble keeping your mind on what you were doing.

$\begin{array}{ll}0 & \text { never } \\ 1 & \text { rarely } \\ 2 & \text { a lot of the time } \\ 3 & \text { most of the time or all of the time } \\ 6 & \text { Refused } \\ 8 & \text { Don't know }\end{array}$

6. You felt depressed.

0 never

1 rarely

2 a lot of the time

3 most of the time or all of the time

6 Refused

8 Don't know

7. You felt you were too tired to do things.

$\begin{array}{ll}0 & \text { never } \\ 1 & \text { rarely } \\ 2 & \text { a lot of the time } \\ 3 & \text { most of the time or all of the time } \\ 6 & \text { Refused } \\ 8 & \text { Don't know }\end{array}$

8. You felt hopeful about the future.

$\begin{array}{ll}0 & \text { never } \\ 1 & \text { rarely } \\ 2 & \text { a lot of the time } \\ 3 & \text { most of the time or all of the time } \\ 6 & \text { Refused } \\ 8 & \text { Don't know }\end{array}$


9. You thought your life had been a failure.

$\begin{array}{ll}0 & \text { never } \\ 1 & \text { rarely } \\ 2 & \text { a lot of the time } \\ 3 & \text { most of the time or all of the time } \\ 6 & \text { Refused } \\ 8 & \text { Don't know }\end{array}$

10. You felt fearful.

$\begin{array}{ll}0 & \text { never } \\ 1 & \text { rarely } \\ 2 & \text { a lot of the time } \\ 3 & \text { most of the time or all of the time } \\ 6 & \text { Refused } \\ 8 & \text { Don't know }\end{array}$

11. You were happy.

$\begin{array}{ll}0 & \text { never } \\ 1 & \text { rarely } \\ 2 & \text { a lot of the time } \\ 3 & \text { most of the time or all of the time } \\ 6 & \text { Refused } \\ 8 & \text { Don't know }\end{array}$

12. You talked less than usual.

$\begin{array}{ll}0 & \text { never } \\ 1 & \text { rarely } \\ 2 & \text { a lot of the time } \\ 3 & \text { most of the time or all of the time } \\ 6 & \text { Refused } \\ 8 & \text { Don't know }\end{array}$


13. You felt lonely.

$\begin{array}{ll}0 & \text { never } \\ 1 & \text { rarely } \\ 2 & \text { a lot of the time } \\ 3 & \text { most of the time or all of the time } \\ 6 & \text { Refused } \\ 8 & \text { Don't know }\end{array}$

14. People were unfriendly to you.

$\begin{array}{ll}0 & \text { never } \\ 1 & \text { rarely } \\ 2 & \text { a lot of the time } \\ 3 & \text { most of the time or all of the time } \\ 6 & \text { Refused } \\ 8 & \text { Don't know }\end{array}$

15. You enjoyed life.

$\begin{array}{ll}0 & \text { never } \\ 1 & \text { rarely } \\ 2 & \text { a lot of the time } \\ 3 & \text { most of the time or all of the time } \\ 6 & \text { Refused } \\ 8 & \text { Don't know }\end{array}$

16. You felt sad.

$\begin{array}{ll}0 & \text { never } \\ 1 & \text { rarely } \\ 2 & \text { a lot of the time } \\ 3 & \text { most of the time or all of the time } \\ 6 & \text { Refused } \\ 8 & \text { Don't know }\end{array}$


17. You felt that people disliked you.

$\begin{array}{ll}0 & \text { never } \\ 1 & \text { rarely } \\ 2 & \text { a lot of the time } \\ 3 & \text { most of the time or all of the time } \\ 6 & \text { Refused } \\ 8 & \text { Don't know }\end{array}$

18. It was hard to get started doing things.

$\begin{array}{ll}0 & \text { never } \\ 1 & \text { rarely } \\ 2 & \text { a lot of the time } \\ 3 & \text { most of the time or all of the time } \\ 6 & \text { Refused } \\ 8 & \text { Don't know }\end{array}$

19. You felt life was not worth living.

$\begin{array}{ll}0 & \text { never } \\ 1 & \text { rarely } \\ 2 & \text { a lot of the time } \\ 3 & \text { most of the time or all of the time } \\ 6 & \text { Refused } \\ 8 & \text { Don't know }\end{array}$




\section{APPENDIX C: Alcohol Use Measure}

1. During the past 12 months, on how many days did you drink alcohol?

$1 \quad$ Every day or almost every day

23 to 5 days a week

31 or 2 days a week

42 or 3 days a month

5 once a month or less (3-12 times in the past 12 months)

61 or 2 days in the past 12 months

$7 \quad$ Never

96 Refused

$97 \quad$ Legitimate skip

98 Don't know

2. Over the past 12 months, on how many days did you drink five or more drinks in a row?

1 never

2 rarely

3 a lot of the time

4 most of the time or all of the time

8 Refused

9 Don't know

96 Refused

97 Legitimate skip

98 Don't know 
VITA

\section{BEVERLY A. RUFFIN}

2000

B.A., Psychology

University of Maryland Baltimore County

Catonsville, MD

2005

M.S., Neuropsychology

Howard University

Washington, D.C

2005-2006 Community Outreach/Research Training Manager

Minority Organ Tissue Transplant Education Program - Howard

University

Washington, D.C.

2006-2008 Research Assistant

National Institute on Alcohol Abuse and Alcoholism - Division of

Epidemiology and Prevention Research

Bethesda, MD

2008-present Research Assistant

Center for Research on U.S. Latino HIV / AIDS and Drug Abuse

Florida International University

Miami, Florida

\section{PUBLICATIONS AND PRESENTATIONS}

De La Rosa, M.R., Sanchez, M., Dillon, F., Ruffin, B.A., \& Schwartz, S.J. (2011). Alcohol use among Latinos: A comparison of pre-immigration, post-immigration, and U.S. born Latinos. Journal of Immigrant Health (In press).

Sanchez, M., Dillon, F., Ruffin, B.A., \& De La Rosa, M.R. (2011). The influence of religious coping on the acculturative stress of recent Latino immigrants. Journal of Ethnic and Cultural Diversity in Social Work (In press).

Faden, V.B., Ruffin, B.A., Newes-Adeyi, G., \& Chen, C. (2010). The relationship among pubertal stage, age, and drinking in adolescent boys and girls. Journal Child \& Adolescent Substance Abuse.

Rojas, P., Ruffin, B.A. Malow, R., Rothe, E.M., Rosenberg, R. (2010). The HIV and Substance Abuse in the Caribbean. In L. Norman, R. Malow, M. Day (Eds) HIV/AIDS and Substance Abuse in the Caribbean: An overlooked epidemic. Benthan Ebooks. 
Sanchez, M., Dillon, F., Ruffin, B.A., De La Rosa, M.R. (2011). The influence of religious coping on the acculturative stress of recent Latino immigrants. Presented at the Annual Conference of the Society for Social Work and Research, Tampa, FL.

Sanchez, M., Dillon, F., Ruffin, B.A., De La Rosa, M.R. (2011). Examining the relationship between religious coping and acculturative stress among recent Latino immigrants. Presented at the American Public Health Association Annual Conference, Washington, D.C. 